NBER WORKING PAPER SERIES

\title{
LINEAR IV REGRESSION ESTIMATORS FOR STRUCTURAL DYNAMIC DISCRETE CHOICE MODELS
}

\author{
Myrto Kalouptsidi \\ Paul T. Scott \\ Eduardo Souza-Rodrigues \\ Working Paper 25134 \\ http://www.nber.org/papers/w25134 \\ NATIONAL BUREAU OF ECONOMIC RESEARCH \\ 1050 Massachusetts Avenue \\ Cambridge, MA 02138 \\ October 2018, Revised February 2020
}

We are grateful to Victor Aguirregabiria, Chris Conlon, Øystein Daljord, Mike Dickstein, Bo Honoré, Rob McMillan, Magne Mogstad, Matthew Osborne, Lucas Lima, Marc-Antoine Schmidt, Yuanyuan Wan, Stan Zin, and seminar participants at various universities for many helpful comments. We also thank the Editor, the Associate Editor, and the anonymous referees. Jan Dee and Walter Zhang provided outstanding research assistance. Much of the material of this paper was formerly part of our paper "Identification of Counterfactuals in Dynamic Discrete Choice Models", NBER Working Paper 21527. All remaining errors are our own. The views expressed herein are those of the authors and do not necessarily reflect the views of the National Bureau of Economic Research.

NBER working papers are circulated for discussion and comment purposes. They have not been peer-reviewed or been subject to the review by the NBER Board of Directors that accompanies official NBER publications.

(C) 2018 by Myrto Kalouptsidi, Paul T. Scott, and Eduardo Souza-Rodrigues. All rights reserved. Short sections of text, not to exceed two paragraphs, may be quoted without explicit permission provided that full credit, including $(\odot$ notice, is given to the source. 
Linear IV Regression Estimators for Structural Dynamic Discrete Choice Models

Myrto Kalouptsidi, Paul T. Scott, and Eduardo Souza-Rodrigues

NBER Working Paper No. 25134

October 2018, Revised February 2020

JEL No. C13,C35,C36,C51,C61

\begin{abstract}
$\underline{\text { ABSTRACT }}$
In structural dynamic discrete choice models, unobserved and mis-measured state variables may lead to biased parameter estimates and misleading inference. In this paper, we show that instrumental variables can address such measurement problems when they relate to state variables that evolve exogenously from the perspective of individual agents (i.e., market-level states). We define a class of linear instrumental variables estimators that rely on Euler equations expressed in terms of conditional choice probabilities (ECCP estimators). These estimators do not require observing or modeling the agent's entire information set, nor solving or simulating a dynamic program. As such, they are simple to implement and computation-ally light. We provide constructive arguments for the identification of model primitives, and establish the estimator's consistency and asymptotic normality. Four applied examples serve to illustrate the ECCP approach's implementation, advantages, and limitations: dynamic demand for durable goods, agricultural land use change, technology adoption, and dynamic labor supply. We illustrate the estimator's good finite-sample performance in a Monte Carlo study, and we estimate a labor supply model empirically for taxi drivers in New York City.
\end{abstract}

Myrto Kalouptsidi

Department of Economics

Harvard University

Littauer 124

Cambridge, MA 02138

and NBER

myrto@fas.harvard.edu

Paul T. Scott

NYU Stern School of Business

Kaufman Management Center, 7-77

New York University

New York, NY 10012

ptscott@gmail.com
Eduardo Souza-Rodrigues

Department of Economics

University of Toronto

Max Gluskin House,

150 St. George Street, room 324

Toronto, ON, Canada

edusouzarod@gmail.com 


\section{Introduction}

Instrumental variables methods are widely used to address omitted variables and measurement error problems in reduced-form models. In this paper, we show that instrumental variables can play the same role in structural dynamic discrete choice (DDC) models, as long as the measurement problems involve market-level state variables (also known as forcing variables), i.e., variables that evolve exogenously from the perspective of individual agents. To that end, we define a class of linear IV regression estimators for structural dynamic discrete choice models, which we call Euler Equations in Conditional Choice Probabilities (ECCP) estimators. ECCP estimators are a discrete-choice analog to the Euler equation approach for models with continuous choice variables developed by Hall (1978), Hansen and Sargent (1980, 1982), and Hansen and Singleton (1982). ${ }^{1}$

Structural DDC models have proven useful in a variety of important fields - for example, in the study of firm dynamics, consumer demand, labor markets, and environmental problems. Standard methods for estimating DDC models require the computation of continuation value functions, by either solving the full dynamic problem (Rust, 1987) or measuring the continuation values by forward-solving (Hotz and Miller, 1993; Pesendorfer and Schmidt-Dengler, 2008) or forwardsimulating (Hotz, Miller, Sanders, and Smith, 1994; Bajari, Benkard, and Levin, 2007). In this context, Arcidiacono and Miller (2011) and Aguirregabiria and Magesan (2013) represent two major departures from prior work, providing strategies for estimating model parameters without calculating continuation values, thereby reducing the computational burden of estimating DDC models substantially. Arcidiacono and Miller (2011) define the finite dependence property and show how it allows the econometrician to construct conditions from an agent's optimization problem in which the continuation values cancel out. ${ }^{2}$ Aguirregabiria and Magesan (2013) propose a representation of the discrete choice problem as a continuous decision problem in which the decision variables are choice probabilities. Based on this representation, they derive first-order conditions for optimization that are expressed in terms of choice probabilities, and that are similar to Euler equations for continuous decision problems.

We build on these methodological contributions to allow for measurement problems in marketlevel state variables, including serially correlated unobserved states, endogeneity problems, and measurement error. It is widely acknowledged that the presence of such unobservables is an important concern in empirical applications, and that ignoring them may lead to biased estimates and misleading inference. Empirical work exploring finite dependence in structural DDC models that allows for such measurement problems has appeared in the recent applied literature (Scott,

\footnotetext{
${ }^{1}$ Euler equation estimators rely on agents' behavior in adjacent time periods, drawing on restrictions that are necessary for dynamic optimization.

${ }^{2}$ Finite dependence requires that, starting from any two different states, there exist two finite sequences of actions that will lead to the same distribution over states. See Altug and Miller (1998) for an early application exploiting finite dependence, and Arcidiacono and Ellickson (2011) for further discussion of the role of finite dependence in DDC models.
} 
2013; Diamond, McQuade, and Qian, 2018; De Groote and Verboven, 2019; Traiberman, 2019). ${ }^{3}$ However, the underlying class of models has yet to receive a comprehensive econometric treatment. This paper aims to fill that gap.

To that end, we provide sufficient conditions for both parametric and nonparametric identification of DDC model primitives. The identification arguments are constructive and lead naturally to an estimator, the ECCP estimator. Like other conditional-choice-probability-based estimators (Hotz and Miller, 1993; Aguirregabiria and Mira, 2002; Pesendorfer and Schmidt-Dengler, 2008), the ECCP estimator involves two steps: first estimating conditional choice probabilities, and then estimating parameters of the model. Unlike other CCP estimators, the second step amounts to estimating a linear regression equation, making it easy to implement and computationally light. ${ }^{4}$ Both steps can be implemented using standard tools available in econometrics software packages such as STATA and R. We establish the consistency and asymptotic normality of the estimator, and we illustrate the finite-sample performance of the ECCP estimator in a Monte Carlo study of dynamic demand for durable goods. We find that it performs well in finite samples while estimation techniques ignoring measurement problems in state variables can be substantially biased. We also illustrate the performance of the ECCP estimator empirically, in the context of dynamic labor supply for taxi drivers in New York City. Here, we find that ignoring endogeneity issues leads to the underestimation of labor supply responses to earnings - consistent with usual biases in simultaneity problems - while the ECCP estimates provide plausible response patterns.

To identify and estimate structural parameters, the ECCP approach exploits moment restrictions implied by dynamic optimization. These moment restrictions can be constructed as long as (a) the state variables can be decomposed into agent-specific state variables and market-level states, and (b) there is finite dependence in the agent-specific state variables. Under these conditions, ECCP equations (like Euler equations in general) involve relationships between current behavior and expected future behavior. By using observed (realized) rather than expected behavior in successive time periods, the ECCP equation can serve as a valid estimating equation. Furthermore, unobservable variables and measurement error can be represented in ECCP equations in the same way that they are represented in standard regression equations, and handled similarly through the use of instrumental variables. ${ }^{5}$

The ECCP approach allows researchers to deal with endogeneity problems using standard linear instrumental variables techniques. No assumptions are needed regarding the evolution of the unobservable shocks, except that they satisfy exclusion restrictions (i.e., they are uncorrelated with

\footnotetext{
${ }^{3}$ Scott (2013) studies how US agricultural policies affect farmers' land use choices; Diamond, McQuade, and Qian (2018) study the welfare impacts of rent control on tenants and landlords; De Groote and Verboven (2019) investigate the adoption of solar photovoltaic systems for electricity production; Traiberman (2019) focuses on workers' occupational reallocations from trade liberalization.

${ }^{4}$ As long as agents' payoffs are linear-in-parameters, the resulting ECCP equation will also be linear-inparameters.

${ }^{5}$ The estimating equations involve CCPs in successive time periods, and so require sufficiently rich panel data so that CCPs can be estimated separately for each cross section.
} 
instrumental variables). For example, cost shifters may be used as instruments for unobserved demand shocks (De Groote and Verboven, 2019); lagged observed states form another possible source of instruments available to researchers; quasi-experiments form yet another source of exogenous variation.

Unlike other approaches to estimating DDC models, the ECCP approach does not require a full model of the agent's information set. This is a significant advantage, as modeling market-level state variables can be both conceptually and computationally demanding in many applications. For instance, it may be difficult for the econometrician to model the evolution of certain state variables (e.g., when government policy is in flux as in De Groote and Verboven (2019)); observed market states may be insufficient to capture the true extent of market heterogeneity (e.g., unobserved local price variation as in Scott (2013)); or it may be difficult to reliably estimate how market-level state variables evolve, as when the dimensionality of the state space is large relative to the length of the panel data (e.g., when the state-space involves industry-level productivity shocks for a variety of industries as in Traiberman (2019)).

Related Literature. This paper relates to several important prior studies examining the identification and estimation of structural DDC models. In addition to the previously mentioned contributions (which focused mostly on estimation), there exists a growing literature on identification that builds on the seminal work of Rust (1994) and Magnac and Thesmar (2002). Arcidiacono and Miller (2019a) investigate the nonparametric identification of DDC models for both stationary and nonstationary environments in the presence of long and short panel data, relying on single-action finite dependence to eliminate continuation values. ${ }^{6}$ Our results build on those of Arcidiacono and Miller (2019a) by (a) allowing for endogeneity problems in market-level state variables, and (b) showing that parametric restrictions commonly imposed in applied work allow researchers to relax the single-action requirement and identify model parameters under general patterns of finite dependence. Blevins (2014) shows how models with discrete and continuous choice variables can be identified in the presence of (observed) continuous states under the conditional independence assumption on the unobservables. Our results can be combined with those of Blevins (2014) to generate an augmented set of moment restrictions that incorporates both the ECCP and the standard Euler equations for continuous variables.

Serially correlated unobserved state variables in structural dynamic models are a widespread problem without a standard econometric solution. Kasahara and Shimotsu (2009), Hu and Shum (2012), Blevins, Khwaja, and Yang (2018), Aguirregabiria, Gu, and Luo (2019), and Berry and Compiani (2019) represent other approaches to estimating DDC models with unobserved state variables. Kasahara and Shimotsu (2009) restrict individual unobserved heterogeneity to have a

\footnotetext{
${ }^{6}$ Single-action finite dependence requires that the same action must be chosen repeatedly finitely many times in order to reset the distribution of the state variables. The empirical papers cited above (Scott, 2013; Diamond, McQuade, and Qian, 2018; De Groote and Verboven, 2019; Traiberman, 2019) make use of one-period finite dependence (specifically, terminal or renewal actions), which is a special case of single-action finite dependence.
} 
discrete distribution that is invariant over time (see also Arcidiacono and Miller, 2011). Hu and Shum (2012) allow the unobserved state to follow a Markovian process, but require it to be a scalar, to have the same cardinality as the action space, and to be realized before the realization of the observed states. Blevins, Khwaja, and Yang (2018) use particle filtering methods to allow for an unobservable state that follows a first-order autoregressive process. Aguirregabiria, Gu, and Luo (2019) extend the conditional likelihood approach (in which the likelihood function conditional on a sufficient statistic does not depend on individual fixed-effects) to estimate structural dynamic logit models with fixed-effects and two endogenous state variables: the lagged decision variable and the time duration in the last choice. Berry and Compiani (2019) allow for serially correlated unobserved states and propose the use of lagged exogenous state variables as instrumental variables for (econometrically) endogenous states, for models with both continuous and discrete actions, and obtain partial identification of payoff function parameters in a discrete choice setting. ${ }^{7}$ Whereas the ECCP approach allows for market-level unobserved heterogeneity very flexibly, these papers allow for individual-level unobserved heterogeneity with stronger restrictions on the nature of that heterogeneity. As such, the ECCP approach complements, and can be combined with, these other contributions.

Models of dynamic demand is one area where dynamic discrete choice models have been estimated while allowing for endogeneity concerns with market-level variables (i.e., prices being correlated with market demand shocks). Existing studies impose strong functional form restrictions on how observed and unobserved state variables evolve (Hendel and Nevo, 2006; Gowrisankaran and Rysman, 2012; Melnikov, 2013). Such restrictions - in particular, inclusive value sufficiency - limit the dimensionality of the state space to render the problem tractable, facilitating an estimation approach that relies on solving agents' dynamic problem. However, such restrictions effectively impose that in two different states (e.g., high price with low unobserved product quality versus low price with high unobserved product quality), consumers must have the same expectations. The ECCP approach avoids both the need to solve the dynamic problem and the need to specify a restrictive process for how state variables evolve in the estimation procedure. ${ }^{8}$

Morales, Sheu, and Zahler (2019) and Dickstein and Morales (2018) pioneered the use of Eulerequation-like estimators for DDC models using moment inequalities. The ECCP approach, like most estimation approaches for DDC models since Rust (1987), relies on the existence of conditionally independent individual payoff shocks with a distribution that is known ex-ante (e.g.,

\footnotetext{
${ }^{7}$ Berry and Compiani (2019) assume the evolution of observed uncontrolled states (i.e., state variables whose evolutions are not affected by individuals' choices) is fully independent of the evolution of unobserved states, so that they can be used as instruments for the observed controlled state variables. The ECCP method, in contrast, does not require such full independence. Berry and Compiani's approach and ours are therefore non-nested.

${ }^{8}$ Recently, and independently, Chou, Derdenger, and Kumar (2019) considered a special case of the ECCP method for demand models, requiring two terminal actions for identification. Although the ECCP approach can accommodate finitely many unobserved types, as in Scott's (2013) use of Arcidiacono and Miller's (2011) strategy, it does not, on its own, accommodate richer unobserved consumer heterogeneity (e.g., continuously distributed random coefficients on flow utilities). We regard extensions of the ECCP approach that incorporate richer forms of consumer heterogeneity as an important avenue for future work.
} 
logit). In contrast, the moment inequalities approach allows researchers to impose less structure on payoff shocks, requiring minimal distributional assuptions on the error term. However, the moment inequalities approach yields only partially identified parameter estimates, and it has only been shown to be robust to a limited set of endogeneity concerns (e.g., when the error terms are fixed effects that can be differenced out; see Pakes (2010)).

The remainder of the paper is organized as follows. Section 2 describes the framework, and introduces four applied examples (dynamic demand for durable goods, land use change, technology adoption, and dynamic labor supply). Section 3 derives the ECCP equations, and illustrates them in the context of the applied examples. Section 4 discusses the identification results, as well as several possible extensions. Section 5 presents the ECCP estimator and establishes its asymptotic properties. Section 6 shows the Monte Carlo evidence, and Section 7 presents the empirical exercise. Section 8 concludes. (All proofs can be found in the Online Appendix.)

\section{Model}

In the model, time is discrete and indexed by $t=1, \ldots, T$, where the time horizon $T$ can be either finite or infinite. There are $N$ agents operating independently in $M$ independent markets, such as geographical locations. Every period $t$, agent $i$ in market $m$ chooses an action $a_{\text {imt }} \in \mathcal{A}=\{0, \ldots, A\}$, $A<\infty$, with the goal of maximizing her expected discounted sum of payoffs

$$
E\left[\sum_{\tau=t}^{T} \beta^{\tau-t} \Pi_{i m \tau} \mid \mathcal{I}_{i m t}\right],
$$

where $\Pi_{i m t}$ denotes per-period payoffs, $\beta \in[0,1)$ is the discount factor, and $E\left[\cdot \mid \mathcal{I}_{i m t}\right]$ denotes the expectation operator conditioned on the information set $\mathcal{I}_{\text {imt }}$ available to agent $i$ in market $m$ at time $t$.

The payoff function $\Pi_{i m t}$ depends on the state variables $\mathbf{s}_{i m t}=\left(k_{i m t}, w_{m t}, \eta_{m t}, \varepsilon_{i m t}\right)$, where $\left(k_{i m t}, w_{m t}\right)$ are observed by the econometrician, while $\left(\eta_{m t}, \varepsilon_{i m t}\right)$ are not. The observed states $k_{i m t} \in \mathcal{K}$ are "controlled": their evolution can be affected by the agent's actions; such states may include a firm's capital stock, size or type of product, lagged decisions, and duration in the last choice. The market-level observed and unobserved states, $w_{m t}$ and $\eta_{m t}$, cannot be affected by the agent's actions; such states may include market demand variables, aggregate input prices, or the government policy environment. We collect these market-level states into the vector $\omega_{m t}=$ $\left(w_{m t}, \eta_{m t}\right) \in \Omega$. We assume $\mathcal{K}$ is finite, as usually done in the literature, but we allow $\omega_{m t}$ to be continuous. Neither assumption is important and our results apply to both discrete and continuous states. Our results do not require us to specify the dimension of $\eta_{m t}$, so there may be many unobserved market-level state variables. Finally, the unobservable state $\varepsilon_{\text {imt }}=\left(\varepsilon_{0 i m t}, \ldots, \varepsilon_{\text {Aimt }}\right)$ is 
i.i.d. across agents and time with a distribution function that is absolutely continuous with respect to the Lebesgue measure in $\mathbb{R}^{A+1}$.

As usual, in each period $t$ agents observe the state variables $\mathbf{s}_{i m t}$, make choices $a_{i m t}$, flow payoffs are then realized, and states evolve. Agent's information set $\mathcal{I}_{\text {imt }}$ therefore includes all current and past state variables $\mathbf{s}_{i m t}$, as well as all past actions.

Assumption 1. (State Transitions) The state variables $\mathbf{s}_{i m t}$ follow a controlled first-order Markov process with transition distribution function that factors as,

$$
F\left(\mathbf{s}_{i m t+1} \mid a, \mathbf{s}_{i m t}\right)=F^{k}\left(k_{i m t+1} \mid a, k_{i m t}, w_{m t}\right) F^{\omega}\left(\omega_{m t+1} \mid \omega_{m t}\right) F^{\varepsilon}\left(\varepsilon_{i m t+1}\right),
$$

where $\omega_{m t}=\left(w_{m t}, \eta_{m t}\right)$. The distribution $F^{\varepsilon}\left(\varepsilon_{i m t+1}\right)$ is known by the econometrician.

Equation (1) limits our focus to settings with small decision makers, as opposed to dynamic games. It says that market-level state variables $\omega_{m t}$ are perceived as exogenous by individual agents, ruling out settings where an individual agent's decision can have aggregate impacts (i.e., $F^{\omega}$ does not depend on individual action $a$ ). It also rules out settings where there are externalities acting through the agent-level state variables $k$, such as when one agent's state depends on a neighbor's decision (i.e., $F^{k}$ for agent $i$ does not depend on actions of agent $j$ ). Further, while equation (1) accomodates impacts of observable states $w_{m t}$ on the evolution of individual-level states $k_{i m t}$, it does not allow for impacts of aggregate unobserved states $\eta_{m t}$ on $k_{i m t}$. Examples in which the agent-level states $k_{i m t}$ do not depend on aggregate states include capital stocks, time duration since last action, lagged choices, among others. It is worth mentioning that Assumption 1 does not require that observable and unobservable states, $w_{m t}$ and $\eta_{m t}$, evolve independently to each other; this allows for (econometric) endogeneity issues, as we discuss below. Finally, the assumption that $F^{\varepsilon}$ is known by the econometrician is standard in the literature; typically, researchers assume $\varepsilon_{i m t}$ follows a type 1 extreme value distribution or a multivariate normal. Note that we do not allow for individual-level unobserved states other than $\varepsilon_{\text {imt }}$; extensions to unobserved heterogeneity are possible and discussed in Remark 2 in Section 3.

Assumption 2. (Discount Factor and Payoff) The discount factor $\beta \in[0,1)$ is known by the econometrician. The per period payoff function is given by,

$$
\Pi\left(a, \mathbf{s}_{i m t}\right)=\bar{\pi}\left(a, k_{i m t}, w_{m t}\right)+\xi\left(a, k_{i m t}, \omega_{m t}\right)+\varepsilon_{a i m t} .
$$

Assuming a known discount factor is also common in the applied literature. In principle, one can relax this assumption by combining our approach with that of Abbring and Daljord (2019) to point-identify $\beta$.

The payoff function is assumed additively separable in the unobservables $\xi$ and $\varepsilon$. While it is standard practice to include additively separable idiosyncratic shocks $\varepsilon$ in dynamic discrete 
choice models (Rust, 1994), the other unobserved term of the payoff function, $\xi$, deserves some discussion. The function $\bar{\pi}(a, k, w)$ depends on observed market-level state variables, $w$, and so $\xi(a, k, \omega)$ captures the impact of unobserved market-level state variables $\eta$ on the flow payoff. For example, in dynamic demand models, this term may capture unobservable product characteristics (Gowrisankaran and Rysman, 2012). In migration decision models, it may capture location-specific unobservable amenities (Bishop, 2012; Diamond, McQuade, and Qian, 2018). The term $\xi$ may also reflect mis-measured profits or unobservable costs (Scott, 2013). In all these applied examples, $\xi$ enters the payoff function additively. Although this is not without loss of generality (as it restricts marginal rates of substitutions between observable and unobservable states), relaxing additivity in payoffs is challenging for point-identification, as we discuss in Remark 3 in Section 3.

It is important to stress that $\xi$ is a function of state variables $k$ and $\omega=(w, \eta)$, and it need not be a state variable itself. That is, the value of $\xi$ at time $t$ may not be a sufficient statistic for the distribution of future values of $\xi$ at time $t+1$. In the background, there are some (potentially) more informative state variables $\omega$ that agents use to form their expectations about the future. Consequently, $\xi$ need not evolve according to a first-order Markov process, while $\omega$ does.

In addition, $\bar{\pi}$ and $\xi$ may be correlated because they may depend on the same state variables $w$, or because $w$ and $\eta$ may not be independent to each other (see Assumption 1). These are reasons to consider the use of instrumental variables to identify and estimate the model. Without loss of generality, we assume that $\xi$ is mean zero, and, to simplify notation, define

$$
\pi \equiv \bar{\pi}+\xi
$$

Next, we introduce four applied examples to illustrate what the $\xi$ term can capture in practice, and we preview the advantages of the ECCP approach in the context of each example.

Example 1. Durable Demand. In our Monte Carlo study, we consider a model of dynamic demand for a durable good. Each period $t$, consumer $i$ in market $m$ decides whether or not to purchase a unit of a durable good, in turn discarding the old version of the good if she already owns a unit. The choice set is $\mathcal{A}=\{b, n b\}$, where $b$ means buying the good, and $n b$ means not buying the good.

Consumer $i$ controls state $k_{i m t} \in\{0,1\}$ where $k_{i m t}=0$ if the consumer does not have a unit of the good at the beginning of time period $t$, and $k_{i m t}=1$ when she already owns it. If the consumer chooses not to buy a new unit of the good $\left(a_{i m t}=n b\right)$ when she already owns it $\left(k_{i m t}=1\right)$, then there is probability $\phi$ of product failure, resulting in $k_{i m t+1}=0$. Formally, state $k$ evolves as follows:

$$
\operatorname{Pr}\left(k_{i m t+1}=1 \mid k_{i m t}, a_{i m t}, w_{m t}\right)= \begin{cases}1 & \text { if } a_{i m t}=b \\ 0 & \text { if } a_{i m t}=n b, k_{i m t}=0 \\ 1-\phi & \text { if } a_{i m t}=n b, k_{i m t}=1\end{cases}
$$


We consider one observed exogenous state, the price of the product $w_{m t}$, and one unobserved exogenous state, a quality shock $\eta_{m t}$. They evolve independently of individual consumers' decisions (i.e., consumers are price-takers), but they are not independent to each other: As usual in demand estimation, prices and unobserved quality shocks correlate. State transitions therefore satisfy Assumption 1.9

The consumer enjoys the following flow utility when purchasing the product:

$$
\pi\left(b, k_{i m t}, \omega_{m t}\right)=\theta_{0}+\theta_{1} w_{m t}+\xi\left(b, k_{i m t}, \omega_{m t}\right)
$$

where $\xi\left(b, k_{i m t}, \omega_{m t}\right)=\eta_{m t}$, i.e., the term $\xi$ equals the product unobserved quality. We impose $\theta_{1}<0$ so that demand slopes down. When not purchasing the product, the consumer enjoys utility

$$
\pi\left(n b, k_{i m t}, \omega_{m t}\right)= \begin{cases}\theta_{0} & \text { if } k_{i m t}=1 \\ 0 & \text { if } k_{i m t}=0\end{cases}
$$

Notice that the $\theta_{0}$ term appears in the utility function when the good is being consumed, either through purchase or because the consumer already owns the good. The parameter $\theta_{0}$ can be interpreted as the flow value of consumption. Note also that we assume $\xi\left(n b, k_{i m t}, \omega_{m t}\right)=0$. Since the demand shock $\eta$ enters into consumption only conditional on purchase, we can interpret $\eta$ as a quality shock that the consumer only cares about when the product is newly purchased, such as the quality of the in-store experience or some non-durable services associated with the durable good. Finally, the utility of not consuming the good is set to zero.

Our Monte Carlo example is a simplified version of more general dynamic demand models; extensions to the general case are straightforward. Other approaches to estimating dynamic demand systems (e.g. Hendel and Nevo (2006); Gowrisankaran and Rysman (2012)) rely on solving a consumer's dynamic problem and imposing strong functional form restrictions on how observed and unobserved state variables evolve. Such restrictions - the so-called "inclusive value sufficiency" limit the dimensionality of the state space to render the problem tractable. However, they effectively impose that in two different states (e.g., high price with low quality versus low price with high quality), consumers must have the same expectations. The ECCP approach avoids both the need to solve the dynamic problem and the need to specify a restrictive process for how state variables evolve.

Example 2. Land Use Change. Scott (2013) adopts this framework to model farmers' land use choices. In every period $t$, farmer $i$ in county $m$ chooses whether to plant crops or not, $\mathcal{A}=\{$ crops, other $\}$. The farmer's controlled state variable is the number of years since the field was last in crops (up to some limit $K$ ), so that $k_{i m t} \in\{0,1, \ldots, K\}$. This field state reflects vegetal

\footnotetext{
${ }^{9}$ Note that if the probability of product failure $\phi$ were a function of the unobserved quality $\eta$, then the transition $F^{k}$ would depend on $\eta$, violating Assumption 1.
} 
cover and the state of the terrain. Formally, $k$ follows a deterministic process: Let $k^{\prime}(a, k)$ denote the next period state given $a$ and $k$, then $k^{\prime}(a, k)=0$ if $a=$ crops, and $k^{\prime}(a, k)=\min \{k+1, K\}$ if $a=$ other.

Market-level state variables include agricultural prices, input costs, government taxes and subsidies, among other relevant variables. Scott assumes farmers are price takers and cannot influence government policies, which implies that $F^{\omega}$ does not depend on individual actions $a$ nor individual states $k$. The farmer's payoff consists of expected returns from the chosen land use and involve both observed (e.g. crop prices and yields), represented by $R$, and unobserved returns and costs, represented by $\xi$. The payoff function can be written as

$$
\pi\left(a, k, \omega_{m t}\right)=\theta_{0}(a, k)+\theta_{1} R\left(a, w_{m t}\right)+\xi\left(a, k, \omega_{m t}\right)
$$

where $\theta_{0}(a, k)$ are switching costs parameters. The land-use switching costs create dynamic incentives for landowners

Expected returns $R$ may be calculated based on input and output prices, but price data may be available only at a regional level, leading to measurement error in returns coming from the unobserved local price variation. The $\xi$ term captures this measurement error in expected returns.

Measurement error can plausibly render $\xi$ serially correlated. High-priced localities in one year are likely to be high-priced localities the next year. The ECCP approach can be implemented in the presence of such serially correlated unobserved local price variation.

Furthermore, in the context of agricultural markets, it is difficult to model the evolution of observed market-level state variables, given the large set of variables that can influence farmers' expected returns (e.g., technological conditions, uncertain government policies, crop stocks, etc.). ${ }^{10}$ As mentioned previously, the ECCP approach does not require specifying what all the relevant market-level state variables are in the decision making process, nor specifying (and estimating) how all such variables evolve.

Example 3. Technology Adoption. De Groote and Verboven (2019) study the adoption of renewable energy technologies for electricity production: the solar photovoltaic (PV) systems. In every period $t$, a household $i$ in region $m$ may either choose to not adopt a photovoltaic system, $a=0$, or it may choose to adopt one of the available PV alternatives, so that $\mathcal{A}=\{0,1, \ldots, A\}$. They assume the controlled state $k_{i m t}$ is a dummy variable equal to zero when no solar panel has been installed, and equal to the type of solar panel when one is already installed, so that $k_{i m t+1}=a_{i m t}$. They also assume the household no longer makes a PV adoption decision when

\footnotetext{
${ }^{10}$ For example, see Wright (2014) for a discussion of how traditional models of competitive storage fail to explain recent grain price movements. Wright argues that changes in government policy are crucial in explaining recent grain market behavior. Modelling and estimating beliefs on policy changes, however, are difficult in practice. Further, Scott's measure of crop returns incorporate the prices of eleven different crops: estimating price evolution using an unrestricted vector autoregression model would then involve 121 parameters, many of which would be difficult to estimate accurately given that crop prices movements tend to be highly correlated across crops over time.
} 
$k_{i m t} \geq 1$. The adoption decision is therefore a terminating state; not adopting provides the option of waiting for when the prices may have decreased or when the subsidies for adoption (or the PV quality) may have increased.

Each alternative $a \neq 0$ is characterized by observable attributes $w_{\text {amt }}$ (including capacity sizes, upfront investment prices, electricity cost savings, benefits from subsidies for adoption), and by the unobserved quality (which can be captured by $\eta$ ). The vector of observed state variables is given by $w_{m t}=\left(w_{1 m t}, \ldots, w_{A m t}\right)$, and the vector of unobserved quality is $\eta_{m t}=\left(\eta_{1 m t}, \ldots, \eta_{A m t}\right) .{ }^{11}$ Individual households are price-takers and not able to influence the evolution of product attributes.

De Groote and Verboven specify a linear-in-parameters flow payoff. Specifically, if no PV has been adopted (i.e., $k=0$ ), the payoff from adopting option $a \neq 0$ is

$$
\pi\left(a, 0, \omega_{m t}\right)=w_{a m t} \theta+\xi\left(a, 0, \omega_{m t}\right)
$$

where $\xi\left(a, 0, \omega_{m t}\right)=\eta_{a m t}$. The payoff of the outside option is set to zero: $\pi\left(0, k, \omega_{m t}\right)=0$ for all $k$ and $\omega_{m t}$.

Given that the adoption subsidies are large (and that their levels change substantially over time), government policy is an important observable state variable. The ECCP approach allows for the estimation of the dynamic model without requiring an explicit specification of the law of motion for government policy. Traditional approaches would either assume that changes in government policy are either fully anticipated or complete surprises (Ryan, 2012; Kalouptsidi, 2018); the ECCP approach can accomodate these assumptions but does not require them for identification and estimation.

Example 4. Dynamic Labor Supply. A primary goal of labor economics is to understand how agents make labor supply decisions. Recent studies have used the DDC framework to investigate the daily labor supply decisions of taxi drivers, taking advantage of available rich data on taxi trips in New York City (Buchholz, 2019; Fréchette, Lizzeri, and Review, 2019; Schmidt, 2019). In our empirical application, we make use of this data to estimate taxi drivers' optimal stopping decisions.

At the beginning of each hour $t$ of day $m$, taxi driver $i$ decides whether to continue working $(a=1)$ or to stop $(a=0)$, so that $\mathcal{A}=\{0,1\}$. The decision to stop is a terminal choice that ends the decision making process for the day. The controlled state variable $k_{i m t}$ is the number of hours the driver has been working during the shift, $k \in \mathcal{K}=\{0,1, \ldots, K\}$. It captures the disutility from fatigue as the shift progresses. This state variable evolves deterministically: $k^{\prime}(k, a)=$ $\min \{k+a, K\}$.

Following the previous studies, we take the market-level observed state, $w_{m t}$, to be the total earnings the driver expects to make during hour $t$. It depends on the the probability of finding a

\footnotetext{
${ }^{11}$ In principle, one can consider additional unobserved states. I.e., the unobserved product qualities can be just a subvector of $\eta_{m t}$.
} 
customer, the basic fare (earnings per minute of driving), the trip length, and the customers' tips. The unobserved state $\eta_{m t}$ captures costs and disutility the driver experiences from factors such as congestions, weather shocks, construction sites, city events, etc., that are not observed by the econometrician and are common to all drivers. These shocks may correlate with observed earnings $w_{m t}$ leading to potential (econometric) endogeneity issues.

Given state $k$, the driver's flow payoff when he decides to continue working $(a=1)$ during hour $t$ is

$$
\pi\left(1, k, \omega_{m t}\right)=\theta_{0}+\theta_{1} w_{m t}+\theta_{2} k+\xi\left(1, k, \omega_{m t}\right),
$$

where $\xi\left(1, k, \omega_{m t}\right)=\eta_{m t}$ for all $k$. We expect $\theta_{1}>0$ (so that supply slopes up) and $\theta_{2}<0$ (reflecting the disutility from fatigue). The payoff when the driver decides to stop is set to zero: $\pi\left(0, k, \omega_{m t}\right)=0$ for all $k$ and $\omega_{m t}$.

The ECCP approach allows for serially correlated unobservable factors affecting the working decisions while not requiring explicit specification for how the unobserved shocks evolve, extending therefore the scope of structural estimation for labor supply models.

\subsection{Value Functions and Choice Probabilities}

To simplify exposition, we now focus on stationary infinite horizon models. Our results apply however to both non-stationary and finite-horizon specifications, with the usual proper adjustments to the framework; see Remark 1 in Section 3.

Let $V\left(\mathbf{s}_{i m t}\right)$ be the value function of the dynamic programming problem, i.e., the expected discounted stream of payoffs under optimal behavior. By Bellman's principle of optimality,

$$
V\left(\mathbf{s}_{i m t}\right)=\max _{a \in \mathcal{A}}\left\{\Pi\left(a, \mathbf{s}_{i m t}\right)+\beta E\left[V\left(\mathbf{s}_{i m t+1}\right) \mid a, \mathbf{s}_{i m t}\right]\right\}
$$

Following the literature, we define the ex ante value function:

$$
V\left(k_{i m t}, \omega_{m t}\right) \equiv \int V\left(k_{i m t}, \omega_{m t}, \varepsilon_{i m t}\right) d F^{\varepsilon}\left(\varepsilon_{i m t}\right)
$$

and the conditional value function:

$$
v_{a}\left(k_{i m t}, \omega_{m t}\right) \equiv \pi\left(a, k_{i m t}, \omega_{m t}\right)+\beta E\left[V\left(k_{i m t+1}, \omega_{m t+1}\right) \mid a, k_{i m t}, \omega_{m t}\right]
$$

The agent's optimal policy is given by the conditional choice probability (CCP) function:

$$
p_{a}(k, \omega)=\int 1\left\{v_{a}(k, \omega)+\varepsilon_{a} \geq v_{j}(k, \omega)+\varepsilon_{j}, \text { for all } j \in \mathcal{A}\right\} d F^{\varepsilon}(\varepsilon)
$$

where $1\{\cdot\}$ is the indicator function. Define the $(A+1) \times 1$ vector of conditional choice probabilities 
$p(k, \omega)=\left\{p_{a}(k, \omega): a \in \mathcal{A}\right\} .{ }^{12}$

Finally, it is worth noting that for any $(a, k, \omega)$, there exists a real-valued function $\psi_{a}($.$) derived$ only from $F^{\varepsilon}$ that satisfies the following equality (Arcidiacono and Miller, 2011, Lemma 1):

$$
V(k, \omega)=v_{a}(k, \omega)+\psi_{a}(p(k, \omega))
$$

Equation (5) states that the ex ante value function $V$ equals the value obtained by choosing $a$ today and optimally thereafter $\left(v_{a}\right)$ plus a correction term $\left(\psi_{a}\right)$, because choosing action $a$ today is not necessarily optimal. When $\varepsilon_{i m t}$ follows the type 1 extreme value distribution, then $\psi_{a}(p(k, \omega))=\gamma-\ln p_{a}(k, \omega)$, where $\gamma$ is the Euler constant. ${ }^{13}$

\section{$3 \quad$ ECCP Equations}

In this section, we derive the ECCP equation in a simple case and discuss the main identification ideas. To that end, we first consider the available data and relevant objects that can be estimated directly from the data. Then, we present two important concepts that we rely on in our identification arguments: expectational errors and the finite dependence property. Based on these concepts, we then derive the ECCP equations, and illustrate them in the context of the four examples discussed above. The objective here is to identify the per-period payoff function $\bar{\pi}$. In Section 4, we present a general ECCP equation and our main identification results; see also Online Appendix ??.

Data. Assume the available data set is $\left\{y_{i m t}=\left(a_{i m t}, k_{i m t}, w_{m t}, z_{m t}\right): i=1, \ldots, N ; m=1, \ldots, M ; t=\right.$ $1, \ldots, T\}$, where the vector $z_{m t}$ consists of instrumental variables, as explained below. For our identification arguments, we assume the joint distribution of $y_{i m t}$ is known.

Even though the market state $\omega_{m t}$ is not fully observed, the conditional choice probabilities $p_{a}\left(k, \omega_{m t}\right)$ for a particular market $m$ and time period $t$ can be estimated given a sufficiently rich cross section of agents within market $m$. That is, if we observe many agents with each value of the individual state within each market and time period, then $p_{a}\left(k, \omega_{m t}\right)$ can be estimated using a simple frequency estimator. The law of motion for the agent-controlled states, $F^{k}\left(k_{i m t+1} \mid a, k_{i m t}, w_{m t}\right)$, can also be estimated using a simple frequency estimator (when it is not known in advance). For our identification results, we treat $p_{a}($.$) and F^{k}($.$) as known objects (which implies \psi_{a}($.$) , for all$ $a$, are known as well). ${ }^{14}$ In contrast, the law of motion for market-level states $F^{\omega}\left(\omega_{m t+1} \mid \omega_{m t}\right)$

\footnotetext{
${ }^{12}$ Choice probabilities are invariant to scale normalizations; here we normalize the scale parameter to one. I.e., we take $\Pi_{a}=\bar{\pi}_{a}+\xi_{a}+\sigma \varepsilon_{a}$, with $\sigma=1$.

${ }^{13}$ The Arcidiacono-Miller Lemma can be derived from the Hotz-Miller inversion (Proposition 1 of Hotz and Miller, 1993). Chiong, Galichon, and Shum (2016) propose a novel approach that can calculate $\psi_{a}$ for a broad set of distributions $F^{\varepsilon}$ (see also Dearing, 2019).

${ }^{14}$ See Online Appendix ?? for a formal justification. In practice, some smoothing across markets or individual states may be needed.
} 
cannot be estimated without strong assumptions. (As state $\omega_{m t}$ is not fully observed, $F^{\omega}$ cannot be estimated directly from the data.)

Expectational Errors. We now define expectational errors (also known as forecast or prediction errors). As will be clear below, expectational errors are useful as they allow us to "dispose" of the actual expectations when identifying and estimating the model.

Definition 1. (Expectational errors) For any function $h(k, \omega)$ and particular realization $\omega^{*} \in \Omega$,

$$
\begin{aligned}
e^{h}\left(k^{\prime}, \omega, \omega^{*}\right) & \equiv E_{\omega^{\prime} \mid \omega}\left[h\left(k^{\prime}, \omega^{\prime}\right) \mid \omega\right]-h\left(k^{\prime}, \omega^{*}\right), \\
e^{h}\left(a, k, \omega, \omega^{*}\right) & \equiv \sum_{k^{\prime}} e^{h}\left(k^{\prime}, \omega, \omega^{*}\right) F^{k}\left(k^{\prime} \mid a, k, w\right),
\end{aligned}
$$

where $k^{\prime}$ and $\omega^{\prime}$ denote next period values for $k$ and $\omega$.

The expectational error $e^{h}\left(k^{\prime}, \omega, \omega^{*}\right)$ is the prediction error of $h\left(k^{\prime}, \omega^{\prime}\right)$ for a particular realized value of the individual state $k^{\prime}$; and $e^{h}\left(a, k, \omega, \omega^{*}\right)$ is the corresponding prediction error conditioned on $k, w$, and $a$, integrating over the realizations of the individual state $k^{\prime}$. An important property of forecast errors is that, under rational expectations, they are mean independent of variables that belong to the agent's information set $\mathcal{I}_{\text {imt }}$ (see Lemma 1 in Section 4). ${ }^{15}$

Finite Dependence. Next, we discuss the finite dependence property, proposed by Arcidiacono and Miller (2011). To do so, we introduce the following notation: let $F_{a m t}^{k}$ be the $K \times K$ transition matrix for market $m$ at time period $t$ with $(n, l)$ element equal to $\operatorname{Pr}\left(k_{i m t+1}=k_{l} \mid a, k_{i m t}=k_{n}, w_{m t}\right)$. We focus our definition on $F^{k}$ because, as explained below, our approach requires that just the individual-level transition process must satisfy finite dependence. Importantly, note that finite dependence is not a behavioral assumption, but a property that the state transition process may or may not satisfy in the data.

Definition 2. (Finite Dependence) A pair of choices $a$ and $j$ satisfies $\tau$-period finite dependence if there exists two sequences of actions $\left(a, a_{1}, \ldots, a_{\tau}\right)$ and $\left(j, j_{1}, \ldots, j_{\tau}\right)$ such that, for all $t$,

$$
F_{a m t}^{k} F_{a_{1} m t+1}^{k} \ldots F_{a_{\tau} m t+\tau}^{k}=F_{j m t}^{k} F_{j_{1} m t+1}^{k} \ldots F_{j_{\tau} m t+\tau}^{k} .
$$

We say that $\tau$-period finite dependence holds for the model if all pairs of actions satisfy $\tau$-period finite dependence. ${ }^{16}$

\footnotetext{
${ }^{15}$ In models in which agents have perfect foresight with respect to $\omega$, there is no prediction error and so $e^{h}(\cdot)=0$. In contrast, when agents have biased beliefs, their conditional expectations do not necessarily coincide with the true (data generating) conditional expectations, which implies $E\left[e^{h}\left(a, k_{i m t}, \omega_{m t}, \omega_{m t+1}^{*}\right) \mid \mathcal{I}_{i m t}\right] \neq 0$.

${ }^{16}$ The terms in the sequences $\left(a, a_{1}, \ldots, a_{\tau}\right)$ and $\left(j, j_{1}, \ldots, j_{\tau}\right)$ depend on the particular initial pair of actions $(a, j)$ chosen; for ease of exposition, we do not incorporate this dependence on the initial pairs into our notation.
} 
The $\tau$-period finite dependence property holds if, starting from any two distributions of individual states at the beginning of time period $t$, there are sequences of actions (not necessarily optimal) that result in the same distribution of state variables at $t+\tau$. Single-action $\tau$-period finite dependence is a special case that requires the sequences of actions to be of the type $(a, J, \ldots, J)$ and $(j, J, \ldots, J)$, for any actions $a$ and $j$, and some action $J$. Exchangeable $\tau$-period finite dependence is another special case requiring two sequences composed of the same set of choices taken in different time periods (but not necessarily with the same total number of times); e.g., $(a, J)$ and $(J, a)$, for a one-period dependence, and $(a, J, a)$ and $(J, a, J)$, for a two-period dependence.

Common special cases of one-period dependence are renewal and terminal actions. Action $J$ is a renewal action if, taking action $J$ in period $t+1$ leads to the same distribution of states at the beginning of time period $t+2$, regardless of which state the agent was in during period $t$. Examples of renewal actions are replacing the bus engine (Rust, 1987), planting crops (Scott, 2013), and choosing occupations (Traiberman, 2019). A terminal action ends the decision making process. Examples of terminal actions include a worker retiring (Rust and Phelan, 1997), a mortgage owner defaulting (Bajari, Chu, Nekipelov, and Park, 2016), and a household adopting a PV system (De Groote and Verboven, 2019). For both renewal and terminal actions, (6) simplifies to

$$
F_{a m t}^{k} F_{J m t+1}^{k}=F_{j m t}^{k} F_{J m t+1}^{k}
$$

for all $t$ and all $a, j$.

Other models require more than one period to eliminate dependence in state variables. For example, Bishop (2012), Coate (2016), and Ma (2019) consider different models of migration decisions with relocation costs and limited memory, so that the utility a household derives from living at some location at time $t+2$ depends on where it was located at the previous period $t+1$, but not on where it was located before that. I.e., dependence on initial choice at $t$ can be broken after two periods, resulting in two-period finite dependence.

Altug and Miller (1998) consider female labor supply with human capital appreciation and depreciation in which full depreciation of human capital occurs if a woman stays out of the workforce for $\tau$ periods, leading to a single-action finite dependence. Khorunzhina and Miller (2019) also rely on single-action finite dependence to study housing choices and fertility decisions.

Applications taking advantage of $\tau$-period exchangeable finite dependence include work on consumers' stock market participation (Khorunzhina, 2013), schooling and work decisions with information frictions (Arcidiacono, Aucejo, Maurel, and Ransom, 2016), smoking with rational addiction and learning (Matsumoto, 2016), and self-employment decisions (Humphries, 2018; GendronCarrier, 2018).

Arcidiacono and Miller $(2011,2019 b)$ define finite dependence in terms of stochastic sequences of actions, which is more general than the deterministic sequences presented in Definition 2 . We extend our results to incorporate stochastic sequences in Online Appendix ??, at the cost of 
more burdensome notation. Allowing for stochastic sequences enlarges the set of models that can be analyzed and estimated. For example, Ransom (2019) exploits a two-period stochastic finite dependence property (using employment probabilities as mixing weights) to study the role of labor market frictions and moving costs in migration behavior of workers. Importantly, (stochastic) finite dependence can be learned from data based on a finite number of steps; see the algorithm proposed by Arcidiacono and Miller (2019b). Learning whether finite dependence holds in the data is important because, while it is conceivable that finite dependence may be satisfied when one takes sufficiently long sequences of stochastic choices to (eventually) reset the distribution of states, this property is not guaranteed to be satisfied in short panels; i.e., finite dependence may hold in the model but not in the data when $\tau>T$. Evidently, our identification arguments assume that finite dependence is satisfied with $\tau<T{ }^{17}$

ECCP equation with one-period finite dependence. We now start the derivation of the ECCP equation. For the sake of exposition, we first focus on deriving it for models with renewal and terminal actions (and so, satisfying one-period finite dependence). In Section 4, we show that the ECCP approach extends to models with $\tau$-period finite dependence more generally.

First, note that by combining the conditional value function (3) with equation (5), we obtain:

$$
\pi\left(a, k_{i m t}, \omega_{m t}\right)=V\left(k_{i m t}, \omega_{m t}\right)-\beta E\left[V\left(k_{i m t+1}, \omega_{m t+1}\right) \mid a, k_{i m t}, \omega_{m t}\right]-\psi_{a}\left(k_{i m t}, \omega_{m t}\right)
$$

where $\psi_{a}(k, \omega)$ is a short-cut notation for $\psi_{a}(p(k, \omega))$. Next, note that the expectation of continuation values $E\left[V\left(k_{i m t+1}, \omega_{m t+1}\right) \mid a, k_{i m t}, \omega_{m t}\right]$ is given by:

$$
\begin{aligned}
& \sum_{k^{\prime}} \int_{\omega^{\prime}} V\left(k^{\prime}, \omega^{\prime}\right) d F^{\omega}\left(\omega^{\prime} \mid \omega_{m t}\right) F^{k}\left(k^{\prime} \mid a, k_{i m t}, w_{m t}\right) \\
= & \sum_{k^{\prime}}\left(E_{\omega^{\prime} \mid \omega_{m t}}\left[V\left(k^{\prime}, \omega^{\prime}\right) \mid \omega_{m t}\right]\right) F^{k}\left(k^{\prime} \mid a, k_{i m t}, w_{m t}\right) \\
= & \sum_{k^{\prime}} V\left(k^{\prime}, \omega_{m t+1}\right) F^{k}\left(k^{\prime} \mid a, k_{i m t}, w_{m t}\right)+e^{V}\left(a, k_{i m t}, \omega_{m t}, \omega_{m t+1}\right),
\end{aligned}
$$

where $e^{V}(\cdot)$ is the expectational error of the value function $V(\cdot)$. Substituting (9) in (8), we obtain the following equation:

$$
\begin{aligned}
& \pi\left(a, k_{i m t}, \omega_{m t}\right)+\beta e^{V}\left(a, k_{i m t}, \omega_{m t}, \omega_{m t+1}\right) \\
= & V\left(k_{i m t}, \omega_{m t}\right)-\beta \sum_{k^{\prime}} V\left(k^{\prime}, \omega_{m t+1}\right) F^{k}\left(k^{\prime} \mid a, k_{i m t}, w_{m t}\right)-\psi_{a}\left(k_{i m t}, \omega_{m t}\right) .
\end{aligned}
$$

${ }^{17}$ There are important classes of models in which finite dependence is not valid. For instance, in discrete choice models in which $k$ depreciates at a constant rate, $k_{i m t+1}=\delta k_{i m t}+a_{i m t}$, where $\delta<1$ is the depreciation rate. Examples include capital stock, human capital, and learning-by-doing (as in Benkard, 2000). Yet, finite dependence can be restored in the absence of depreciation rate, $\delta=1$, or when the depreciation is stochastic, $k_{i m t+1}=k_{i m t}+a_{i m t}-f_{i m t}$, where $f_{i m t}$ is a random shock, as in Besanko, Doraszelski, Kryukov, and Satterthwaite $(2010)$. 
Here, we make use of realized values of agents' future expected payoffs $V\left(k^{\prime}, \omega_{m t+1}\right)$ as a noisy measure of agents' expected future payoffs. The use of realized values allows us to relax typical assumptions about how agents form beliefs about the evolution of the (market-level) state variables.

Next, we simplify the notation and use $(m, t)$ subscripts to denote functions that depend on $\omega_{m t}$. We rewrite payoffs as $\pi_{m t}(a, k) \equiv \pi\left(a, k, \omega_{m t}\right)$, while $\bar{\pi}_{m t}(a, k), \xi_{m t}(a, k), V_{m t}(k), p_{a m t}(k)$ and $\psi_{\text {amt }}(k)$ are similarly defined. We also make use of matrix notation, so that $\pi_{a m t}$ is a $K \times 1$ vector that stacks $\pi_{m t}(a, k)$ for all $k \in \mathcal{K}$ (and similarly for the vectors $V_{m t}, p_{\text {amt }}$ and $\psi_{\text {amt }}$ ). Therefore, (10) in matrix form becomes:

$$
\pi_{a m t}+\beta e_{a m, t, t+1}^{V}=V_{m t}-\beta F_{a m t}^{k} V_{m t+1}-\psi_{a m t}
$$

for all $a$, where $e_{a m, t, t+1}^{V}$ stacks $e_{m t, t+1}^{V}(a, k) \equiv e^{V}\left(a, k, \omega_{m t}, \omega_{m t+1}\right)$ for all $k \in \mathcal{K}$, and recall that $F_{a m t}^{k}$ is the $K \times K$ transition matrix for $k$ in market $m$ at time period $t$.

We now eliminate the continuation values $V_{m t}$ and $V_{m t+1}$ from (11) to obtain our regression equation. First, note that the time- $t$ value function term, $V_{m t}$, can be removed by simply differencing equation (11) across two different actions, $a$ and $j$ :

$$
\psi_{j m t}-\psi_{a m t}=\pi_{a m t}-\pi_{j m t}+\beta\left(e_{a m, t, t+1}^{V}-e_{j m, t, t+1}^{V}\right)-\beta\left(F_{j m t}^{k}-F_{a m t}^{k}\right) V_{m t+1} .
$$

Equation (12) states that the difference in utilities the agent obtains by choosing $a$ today and optimally thereafter versus choosing $j$ today and optimally in the future equals the difference between $\psi_{j m t}$ and $\psi_{a m t}$. This can be viewed as an indifference condition: when the realized idiosyncratic shock $\varepsilon_{\text {jimt }}-\varepsilon_{\text {aimt }}$ is smaller than the difference in utilities on the right-hand side of (12), the agent prefers choosing $a$ today and optimally afterwards than $j$ today and optimally later (see equation (4)); when the realization of $\varepsilon_{\text {jimt }}-\varepsilon_{\text {aimt }}$ is greater than the right-hand side of (12), she prefers action $j$ over $a$ (and then optimally in the future); and when the realization of $\varepsilon_{\text {jimt }}-\varepsilon_{\text {aimt }}$ equals $\psi_{\text {jmt }}-\psi_{\text {amt }}$, the agent is indifferent between the two sequences of choices. The term $\psi_{j m t}-\psi_{\text {amt }}$ can therefore be viewed as providing the agent's "indifferent" type.

Next, we rewrite (12) by separating payoffs into the observable and unobservable components:

$$
\begin{aligned}
\psi_{j m t}-\psi_{a m t}= & \bar{\pi}_{a m t}-\bar{\pi}_{j m t}+\xi_{a m t}-\xi_{j m t}+\beta\left(e_{a m, t, t+1}^{V}-e_{j m, t, t+1}^{V}\right) \\
& -\beta\left(F_{j m t}^{k}-F_{a m t}^{k}\right) V_{m t+1} .
\end{aligned}
$$

Note that in the static case where $\beta=0$, equation (13) simplifies to

$$
\psi_{j m t}-\psi_{a m t}=\bar{\pi}_{a m t}-\bar{\pi}_{j m t}+\xi_{a m t}-\xi_{j m t} .
$$

This is a standard logistic regression when $\varepsilon$ has a type 1 extreme value distribution (Berry, 1994). In the static logit model, the left hand side of (14) is the log odds ratio of the choice 
probabilities, $\psi_{j m t}-\psi_{a m t}=\ln \left(\frac{p_{a m t}}{p_{j m t}}\right)$. The right-hand side includes the payoff function $\bar{\pi}$ and the unobservables $\xi$. If the payoffs for one reference action were known or pre-specified (e.g., set $\bar{\pi}_{j m t}=0$, which is common practice), then $\bar{\pi}_{a m t}$ can be estimated using standard methods for regression models. When the unobservables $\xi$ correlate with $w$ (which is an argument of $\bar{\pi}_{a m t}$ ), then we need instruments for $w$, and we can estimate the model parameters using standard instrumental variables techniques.

In the dynamic model, we still need to handle the time- $(t+1)$ value function term, $V_{m t+1}$, before (13) can be used for identification and estimation. As shown by Arcidiacono and Miller (2011), continuation values can be eliminated when the finite dependence property holds.

Let $J$ be a renewal or terminal action. If we recursively substitute $V_{m t+1}$, in equation (13), we obtain

$$
\begin{aligned}
\psi_{j m t}-\psi_{a m t}= & \bar{\pi}_{a m t}-\bar{\pi}_{j m t}+\xi_{a m t}-\xi_{j m t}+\beta\left(e_{a m, t, t+1}^{V}-e_{j m, t, t+1}^{V}\right) \\
& -\beta\left(F_{j m t}^{k}-F_{a m t}^{k}\right)\left(\bar{\pi}_{J m t+1}+\xi_{J m t+1}+\psi_{J m t+1}\right),
\end{aligned}
$$

because the $V_{t+2}$ portions of the value function cancel conditional on action $J$ being chosen in period $t+1$. $^{18}$

One can view (15) as an Euler equation. Intuitively, much like in equation (12), the equality in (15) reflects an indifference condition: When the realization of $\varepsilon_{\text {jimt }}-\varepsilon_{\text {aimt }}$ equals $\psi_{\text {jmt }}-$ $\psi_{\text {amt }}$, the agent is indifferent between choosing a today, $J$ tomorrow and optimally in the future versus choosing $j$ today, $J$ tomorrow and optimally thereafter. This is similar to traditional Euler equations, in which perturbations on choices in adjacent time periods followed by optimal choices in the future in ways that keep the agent indifferent express intertemporal first-order conditions implied by optimal dynamic behavior. Even though it is not immediately obvious that (15) can be viewed as a first-order condition, Aguirregabiria and Magesan (2013) has shown that if we treat the choice probabilities as choice variables themselves, we could derive equation (15) as a firstorder condition, formalizing the analogy between (15) and traditional Euler equations for dynamic problems with continuous choice variables. ${ }^{19}$

Finally, by rearranging (15) we obtain

$$
\psi_{j m t}-\psi_{a m t}+\beta\left(F_{j m t}^{k}-F_{a m t}^{k}\right) \psi_{J m t+1}=\bar{\pi}_{a m t}-\bar{\pi}_{j m t}-\beta\left(F_{j m t}^{k}-F_{a m t}^{k}\right) \bar{\pi}_{J m t+1}+u_{a j m t},
$$

\footnotetext{
${ }^{18}$ Formally, use (11) for $J$ in $t+1$ to solve for $V_{m t+1}$ and replace the latter in (11) for any $a$ in $t$ :

$$
\begin{aligned}
\pi_{a m t}+\beta e_{a m, t, t+1}^{V}= & V_{m t}-\psi_{a m t} \\
& -\beta F_{a m t}^{k}\left[\pi_{J m t+1}+\beta F_{J m t+1}^{k} E_{t+1}\left[V_{m t+2}\right]+\psi_{J m t+1}\right] .
\end{aligned}
$$

Next, evaluate the above at $a$ and $j$ and subtract to obtain (15) using property (7).

${ }^{19}$ Aguirregabiria and Magesan (2013) do not allow for unobserved state variables as we do, but their approach to deriving Euler equations by treating choice probabilities as choice variables could be applied in our setting.
} 
where the econometric error term is $u_{\text {ajmt }}=\widetilde{\xi}_{\text {ajmt }}+\widetilde{e}_{\text {ajmt }}^{V}$, with

$$
\begin{aligned}
& \widetilde{\xi}_{a j m t}=\left(\xi_{a m t}-\xi_{j m t}\right)+\beta\left(F_{a m t}^{k}-F_{j m t}^{k}\right) \xi_{J m t+1}, \\
& \widetilde{e}_{a j m t}^{V}=\beta\left(e_{a m, t, t+1}^{V}-e_{j m, t, t+1}^{V}\right) .
\end{aligned}
$$

The Euler equation (16) can be used to construct moment restrictions that are well-suited for identification and estimation of the payoff function $\bar{\pi}$. Compared to the static model (see the logistic regression in equation (14)), equation (16) adds some terms that "correct" for agents' dynamic behavior. Once the adjustment terms are incorporated into the equation, we can estimate a regression function that is similar to the static logistic model to recover the parameters of interest.

To summarize, in addition to Assumptions 1 and 2, the main ingredients of the ECCP approach to identify and estimate the payoff function are: (a) the finite dependence property on individuallevel states $k$ to derive the Euler equation itself, (b) valid instruments to identify the regression function on the right-hand-side of (16), and (c) pre-specifying the payoff function for some reference action, as usual in discrete choice models, in order to disentangle the individual flow payoffs in the regression function.

Next, we return to each of our applied examples, illustrating the regression equations from the ECCP equation (16) in each context. For the first example, we also outline the steps involved in the derivation of the ECCP equation, as the derivation for the special case is considerably simpler than the general derivation above.

Example 1 (continued). Durable Demand. Recall that the choice set is $\mathcal{A}=\{b, n b\}$, where $b$ means buying the good, and $n b$ means not buying the good, and that the consumer controls state $k_{i m t} \in\{0,1\}$ where $k_{i m t}=0$ if the consumer does not have a unit of the good at the beginning of time period $t$, and $k_{i m t}=1$ when she already owns it. This means that the action of buying the product, $a_{i m t}=b$, is a renewal action, and so $F^{k}$ satisfies one-period finite dependence.

Assume logit errors. We begin with the Hotz-Miller inversion, or equation (5) differenced across the two actions:

$$
\ln \left(\frac{p_{b}\left(k_{i m t}, \omega_{m t}\right)}{p_{n b}\left(k_{i m t}, \omega_{m t}\right)}\right)=v_{b}\left(k_{i m t}, \omega_{m t}\right)-v_{n b}\left(k_{i m t}, \omega_{m t}\right) .
$$

In estimating this model, it suffices to focus on $k_{i m t}=0$. Notice that when $k_{i m t}=0$ (when the agent does not own a unit of a good), the state variable $k_{i m t}$ is a deterministic function of $a_{i m t}$. (This simplifies the derivation of the Euler equation substantially.) We expand the conditional value function given $k_{i m t}=0$, and introduce expectational errors, as follows:

$$
\begin{aligned}
\ln \left(\frac{p_{b}\left(0, \omega_{m t}\right)}{p_{n b}\left(0, \omega_{m t}\right)}\right)= & \theta_{0}+\theta_{1} w_{m t}+\xi_{m t}+\beta\left(V\left(1, \omega_{m t+1}\right)-V\left(0, \omega_{m t+1}\right)\right) \\
& +\beta\left(e^{V}\left(1, \omega_{m t}, \omega_{m t+1}\right)-e^{V}\left(0, \omega_{m t}, \omega_{m t+1}\right)\right)
\end{aligned}
$$

We now exploit the fact that purchasing the good is a renewal action, resulting in the state 
$k^{\prime}=1$ regardless of what the initial state $k$ is. As a result, when we substitute for $V\left(1, \omega_{m t+1}\right)$ and $V\left(0, \omega_{m t+1}\right)$ in equation (19) using equation $(5)$, the time- $(t+2)$ value functions cancel, leaving

$$
\begin{aligned}
\ln \left(\frac{p_{b}\left(0, \omega_{m t}\right)}{p_{n b}\left(0, \omega_{m t}\right)}\right)= & \theta_{0}+\theta_{1} w_{m t}+\xi_{m t}+\beta\left[-\ln p_{b}\left(1, \omega_{m t+1}\right)+\ln p_{b}\left(0, \omega_{m t+1}\right)\right] \\
& +\beta\left(e^{V}\left(1, \omega_{m t}, \omega_{m t+1}\right)-e^{V}\left(0, \omega_{m t}, \omega_{m t+1}\right)\right) .
\end{aligned}
$$

Equation (20) is the Euler equation for this model, and we can construct a regression equation by rearranging it to have all the choice probabilities on the left-hand side:

$$
Y_{m t}=\theta_{0}+\theta_{1} w_{m t}+u_{m t}
$$

where

$$
Y_{m t}=\ln \left(\frac{p_{b}\left(0, \omega_{m t}\right)}{p_{n b}\left(0, \omega_{m t}\right)}\right)+\beta \ln \left(\frac{p_{b}\left(1, \omega_{m t+1}\right)}{p_{b}\left(0, \omega_{m t+1}\right)}\right)
$$

and

$$
u_{m t}=\xi_{m t}+\beta\left(e^{V}\left(1, \omega_{m t}, \omega_{m t+1}\right)-e^{V}\left(0, \omega_{m t}, \omega_{m t+1}\right)\right)
$$

Note that, compared to a static logistic regression, we just need to add an adjustment term to the dependent variable involving the probability of buying the product at $t+1$, and incorporate the expectational errors to the econometric error term. Given an instrumental variable that is correlated with the price $w_{m t}$ but not with the error term $u_{m t}$, we can estimate equation (21) using a linear instrumental variables regression.

Example 2 (continued). Land Use Change. Farmers decide whether to plant crops or not, $\mathcal{A}=\{$ crops, other $\}$, and the controlled state variable $k$ equals the number of years since the field was last in crops. This implies that $a=$ crops is a renewal action, resetting $k$ to zero.

Assuming logit errors, equation (16) can be rewritten for each $k$ as

$$
Y_{m t}(k)=\widetilde{\theta}_{0}(k)+\theta_{1}\left[R\left(\text { crops }, w_{m t}\right)-R\left(\text { other }, w_{m t}\right)\right]+u_{m t},
$$

where

$$
\begin{aligned}
Y_{m t}(k)= & \ln \left(\frac{p_{\text {crops }, m t}(k)}{p_{\text {other }, m t}(k)}\right)+\beta \ln \left(\frac{p_{\text {crops }, m t+1}(0)}{p_{\text {crops }, m t+1}\left(k^{\prime}(\text { other }, k)\right)}\right) \\
\widetilde{\theta}_{0}(k)= & \theta_{0}(\text { crops }, k)-\theta_{0}(\text { other }, k) \\
& +\beta\left(\theta_{0}(\text { crops }, 0)-\theta_{0}\left(\text { crops }, k^{\prime}(\text { other }, k)\right)\right), \\
u_{m t}= & \xi_{m t}(\text { crops }, k)-\xi_{m t}(\text { other }, k) \\
& +\beta\left(\xi_{m t+1}(\text { crops }, 0)-\xi_{m t+1}\left(\text { crops }, k^{\prime}(\text { other }, k)\right)\right) \\
& +\beta\left(e_{m, t, t+1}^{V}(0)-e_{m, t, t+1}^{V}\left(k^{\prime}(\text { other }, k)\right)\right) .
\end{aligned}
$$


Again, given estimates of CCPs, one can construct $Y_{m t}(k)$ and estimate $\widetilde{\theta}_{0}(k)$ and $\theta_{1}$ using linear IV regressions. Under the assumption that $\theta_{0}$ (other, $\left.k\right)=0$ for all $k$, we can recover the switching costs parameters $\theta_{0}($ crops,$k)$ from the regression equation intercepts $\widetilde{\theta}_{0}(k)$.

Example 3 (continued). Technology Adoption. Households choose to adopt one of the available PV alternatives, or to not adopt any. Given that all adoption choices $a>0$ are terminal actions, (16) can be established for any $a>0$ given $k=0$. Here we take $j=0$ and use $J=1$ as the terminal action in period $t+1$ to obtain for any $a \geq 2$,

$$
Y_{a m t}=\left(w_{a m t}-\beta w_{1 m t+1}\right) \theta+u_{a 0 m t}
$$

where

$$
Y_{a m t}=\ln \left(\frac{p_{a m t}(0)}{p_{0 m t}(0)}\right)-\beta \ln p_{1 m t+1}(0),
$$

and

$$
u_{a 0 m t}=\xi_{a m t}-\beta \xi_{1 m t+1}+\beta\left(e_{m, t, t+1}^{V}(a)-e_{m, t, t+1}^{V}(0)\right),
$$

noting that there is no time- $t+1$ choice probability term corresponding to $k_{i m t+1}=a$ in the definition of $Y_{a m t}$ because no decision is made once a PV system has been installed; i.e., $p_{1 m t+1}(k)=$ 0 for $k \geq 1 .^{20}$

As usual, prices may correlate with unobserved quality $\xi_{a m t}$ (which are part of the error term $\left.u_{a 0 m t}\right)$; one needs therefore to instrument for prices to estimate the model parameters. De Groote and Verboven (2019) use the prices of Chinese modules as instruments, arguing that these prices are plausibly exogenous to demand shocks in the Belgian market (and therefore a valid instrument) and that the modules are an important component of solar PV installation costs (therefore, making module prices a strong instrument).

Example 4 (continued). Dynamic Labor Supply. Taxi drivers decide when to stop working, implying that action $a=0$ is a terminal choice. Focusing on drivers who have been working for at least one hour (recall that we are not modeling the decision to start the shift), and assuming logit errors, equation (16) can be written for each $k \geq 1$ as

$$
Y_{m t}(k)=\theta_{0}+\theta_{1} w_{m t}+\theta_{2} k+u_{m t},
$$

where

$$
Y_{m t}(k)=\ln \left(\frac{p_{1 m t}(k)}{p_{0 m t}(k)}\right)+\beta \ln p_{0 m t+1}(k+1)
$$

\footnotetext{
${ }^{20}$ In deriving the regression equation with terminal actions, it is convenient to assume that the idiosyncratic errors have a mean-zero distribution, e.g., the standard type-1 extreme value distribution demeaned by Euler's constant. As a result, the expected flow payoffs in the terminal state do not depend on whether or not the agent still receives an idiosyncratic shock in that state.
} 
and

$$
u_{m t}=\xi_{m t}+\beta e_{m, t, t+1}^{V}(k+1),
$$

noting that, in contrast to the technology adoption case, the $t+1$ terms drop from the regression equation because the agent's continuation value equals zero once the terminal action is chosen. In addition, in the last decision period, the future CCP drops from the definition of $Y_{m t}(k)$ given that there is no more decision to make in the terminal period.

As mentioned previously, the expected hourly earnings $w_{m t}$ can be correlated with the unobservable factors $\xi_{m t}$. In our application, we consider two instruments for $w_{m t}$. The first instrument is the (within-day) lagged earnings, $w_{m t-1}$. Using lagged regressors as instruments is standard practice in panel data models; validity of this instrument requires restrictions on the serial correlation of the econometric error term. The second is a "Hausman-type" of instrument: the hourly earnings in the same hour of the day $t$ but in the previous day $m-1$; i.e., $w_{m-1, t}$. This instrument, which takes the price of the same service in a different market, is valid when the unobservables are independent across markets (even when they are serially correlated within markets) - see Hausman (1996) and Nevo (2000).

Before proceeding to the general case, three remarks are in order.

Remark 1. (Finite-horizon and Nonstationary Models.) Note that the Euler equation (16) holds regardless of the agents' time horizons. The ECCP method can therefore be implemented in models with finite-horizons (as in our taxi drivers example above). Further, by noting that the time index $t$ can also be a component of the observed state $w_{m t}$, our approach can be extended to nonstationary models in which payoffs and observed state transitions can depend directly on the time index $t$.

Remark 2. (Unobserved Heterogeneity.) The ECCP approach can incorporate individual-level unobserved heterogeneity by following the strategies proposed by Kasahara and Shimotsu (2009) and Hu and Shum (2012). They provide conditions to identify type-specific conditional choice probabilities and transition functions for finitely-many unobserved types in a first step. After typespecific CCPs and (agent-level) transition functions are identified in each market $m$ and time period t, equation (16) can be used to identify and estimate the payoffs of each individual-level unobserved type, complementing therefore the ECCP approach.

Remark 3. (Additive Separability in Payoffs.) Assumption 2 requires additive separability in flow payoffs, $\pi=\bar{\pi}+\xi$, which severely restricts marginal rates of substitutions between observable and unobservable states. One reasonable alternative is to assume monotonicity in payoffs, i.e., assume $\pi(a, k, w, \xi)$ is strictly increasing in $\xi$ (in the same manner as in the static nonseparable model of Matzkin, 2003). However, this monotonicity condition implies that the regression function in (16) would then include too many unobservables in a nonlinear way ( $\xi_{\text {amt }}, \xi_{j m t}, \xi_{\text {Jmt }+1}$, and the expectational error $\left.e^{V}\right)$. Given that the unobservables cannot be combined into a single-index term, 
we cannot exploit the strategies proposed by Matzkin (2003) or Chernozhukov and Hansen (2005) to identify the payoff function. (The same difficulties arise if we drop Assumption 1 and allow $F^{k}$ to depend on unobservable states.) Identifying the structural parameters $\bar{\pi}$ in such settings remains an open question.

\section{Identification}

We now discuss the identification of the payoff function $\bar{\pi}$ under more general forms of finite dependence. We start the discussion presenting our main propositions, then we turn to the conditions to obtain valid instruments (Section 4.1), and we close the section with three relevant extensions to the ECCP framework (Section 4.2).

Take two sequences of actions $\left(a, a_{1}, \ldots, a_{\tau}\right)$ and $\left(j, j_{1}, \ldots, j_{\tau}\right)$ satisfying $\tau$-period finite dependence (see Definition 2). ${ }^{21}$ Extending equation (16) to $\tau$-period dependence, we obtain the following equation (see Online Appendix ?? for a detailed derivation):

$$
\begin{aligned}
& \psi_{j m t}-\psi_{a m t}+F_{j m t}^{k} \sum_{d=1}^{\tau} \beta^{d} \Lambda_{j m t d} \psi_{j_{d} m t+d}-F_{a m t}^{k} \sum_{d=1}^{\tau} \beta^{d} \Lambda_{a m t d} \psi_{a_{d} m t+d} \\
& =\bar{\pi}_{a m t}-\bar{\pi}_{j m t}+F_{a m t}^{k} \sum_{d=1}^{\tau} \beta^{d} \Lambda_{a m t d} \pi_{a_{d} m t+d}-F_{j m t}^{k} \sum_{d=1}^{\tau} \beta^{d} \Lambda_{j m t d} \pi_{j_{d} m t+d} \\
& +u_{a j m t}
\end{aligned}
$$

where the $K \times K$ matrix $\Lambda_{a m t d}$ is observed (estimable), and is defined recursively

$$
\begin{array}{llcl}
\Lambda_{a m t d}= & I, & \text { for } d=1, \\
\Lambda_{a m t d}= & \Lambda_{a m t, d-1} F_{a_{d-1} m t+d-1}^{k}, & \text { for } d \geq 2,
\end{array}
$$

and the econometric error term is now $u_{a j m t}=\widetilde{\xi}_{a j m t}+\widetilde{e}_{\text {ajmt }}^{V}$, with

$$
\begin{aligned}
\widetilde{\xi}_{a j m t}= & \xi_{a m t}+F_{a m t}^{k} \sum_{d=1}^{\tau} \beta^{d} \Lambda_{a m t d} \xi_{a_{d} m t+d} \\
& -\xi_{j m t}-F_{j m t}^{k} \sum_{d=1}^{\tau} \beta^{d} \Lambda_{j m t d} \xi_{j_{d} m t+d} \\
\widetilde{e}_{a j m t}^{V}= & \beta e_{a m, t, t+1}^{V}+F_{a m t}^{k} \sum_{d=1}^{\tau} \beta^{d+1} \Lambda_{a m t d} e_{a_{d}, m, t+d, t+d+1}^{V} \\
& -\beta e_{j m, t, t+1}^{V}-F_{j m t}^{k} \sum_{d=1}^{\tau} \beta^{d+1} \Lambda_{j m t d} e_{j_{d}, m, t+d, t+d+1}^{V} .
\end{aligned}
$$

\footnotetext{
${ }^{21}$ Recall that the sequences depend on the particular initial pair of actions $(a, j)$ chosen. That is, we use $a_{d}$ to denote the $d$-th action following the initial action $a$ when the alternative initial action is $j$.
} 
As discussed in the context of the applied examples, to identify and estimate the model parameters based on the regression equation (27), it is key to access valid and relevant instrumental variables. We therefore assume the researcher has access to such instruments.

Assumption 3. (Instrumental Variables) There exist instruments $z_{m t}$ such that:

(i) For all functions $q\left(w_{m t}\right)$ with finite expectation, if $E\left[q\left(w_{m t}\right) \mid z_{m t}\right]=0$ almost surely, then $q\left(w_{m t}\right)=0$ almost surely,

(ii) $E\left[\widetilde{\xi}_{\text {ajmt }} \mid z_{m t}\right]=0$, for all a and $j$, and

(iii) $E\left[\widetilde{e}_{\text {ajmt }}^{V} \mid z_{m t}\right]=0$, for all $a$ and $j$.

Assumption 3.(i) is the well-known "completeness condition," which is the nonparametric analog of the standard rank condition for linear models (Newey and Powell, 2003). Assumptions 3.(ii) and 3.(iii) are usual exclusion restrictions, requiring mean independence between the instruments and both the structural errors $\xi$ and the expectational errors $e^{V}$. We discuss instrument validity in the next subsection, after presenting our main propositions.

Proposition 1. Suppose Assumptions 1, 2, and 3 hold. Assume that:

(i) For all pair of actions $a$ and $j$, the single-action $\tau$-period finite dependence property, with $\tau<T$, holds for the agent-level transition $F^{k}$ and for action $J$.

(ii) The payoff $\bar{\pi}(J, k, w)$ is known for all $(k, w)$.

Then, given the joint distribution of observables $y_{i m t}=\left(a_{i m t}, k_{i m t}, w_{m t}, z_{m t}\right)$, the flow payoff $\bar{\pi}(a, k, w)$ is identified for all $(a, k)$ and almost all $w$.

Proposition 1 states that the primitive of interest $\bar{\pi}$ is nonparametrically identified provided that single-action finite dependence holds for some action $J$ and that the flow payoff is known for that same action. Arcidiacono and Miller (2019a) have obtained identification results under similar conditions for nonstationary models with short panels, but ignoring serially correlated unobservables and endogeneity problems.

Intuitively, the regression function on the right-hand-side of (27) is nonparametrically identified in the presence of valid instruments. When action $J$ is choosen repeatedly ( $\tau$ times) to reset the distribution of states, the regression function in (27) becomes a function of the payoffs $\pi_{a m t}, \pi_{j m t}$, and $\pi_{J m t+d}$, for $d=1, \ldots, \tau$. By taking $j=J$ and assuming $\pi_{J m t}$ is known for all states, one can recover the only remaining unknow in the regression function: the flow payoff $\pi_{a m t}$, for all $a \neq J$.

Proposition 1 has two limitations. In general, structural dynamic discrete choice models require restrictions on the payoff function for identification (Rust, 1994; Magnac and Thesmar, 2002). In the literature, identification typically relies on a restriction of the form $\pi(J, \mathbf{s})=0$ for all states 
s and an arbitrary action $J .^{22}$ Proposition 1, however, requires such a restriction on a specific action $J$; this is an unusually strong requirement in the dynamic discrete choice literature. For instance, in a setting with a renewal or terminal action, Proposition 1 requires that the renewal or terminal action's payoffs to be known (i.e., restricted ex-ante). While this is satisfied in our taxi labor supply example (as the flow utility obtained when stop working - a terminal choice is set to zero), the other three applied examples presented above do not impose this restriction on payoffs: In the durable demand example, we do not impose known flow utility when consumers buy the durable good (noting that buying the good is a renewal action); likewise, the switching costs parameters of the land use model are set to zero for the "noncrops" action (while the "crops" choice is renewal); and none of the payoffs obtained from installing a PV system are assumed known by De Groote and Verboven (even though all such choices are terminal actions). This means that, while sufficient for identification, assuming $\bar{\pi}(J, k, w)$ is known for the specific action $J$ is not necessary for identification.

Similarly, while single-action finite dependence is part of the sufficient conditions for identification in Proposition 1, it is not necessary. The next proposition shows that, at the cost of imposing parametric restrictions on payoffs, identification can be obtained under a more general notion of finite dependence, and it does not require the payoff of a specific action $J$ to be known. There exists therefore a clear trade-off between the identification of parametric and nonparametric models in the present context.

Proposition 2. Suppose Assumptions 1 and 2 hold. Assume $\tau$-period finite dependence holds for the agent-level transition process $F^{k}$, with $\tau<T$. Assume also a linear-in-parameters flow payoff: $\bar{\pi}(a, k, w)=x(a, k, w) \theta$, where $\theta \in \mathbb{R}^{P}$, and $x(a, k, w)$ is a known (vector) function. Let $X_{a m t}$ be a $K \times P$ matrix with elements given by $x\left(a, k, w_{m t}\right)$, so that $\bar{\pi}_{a m t}=X_{a m t} \theta$, and define

$$
\begin{aligned}
\widetilde{X}_{a j m t} \equiv & X_{a m t}+F_{a m t}^{k} \sum_{d=1}^{\tau} \beta^{d} \Lambda_{a m t d} X_{a_{d} m t+d} \\
& -X_{j m t}-F_{j m t}^{k} \sum_{d=1}^{\tau} \beta^{d} \Lambda_{j m t d} X_{j_{d} m t+d} .
\end{aligned}
$$

Denote the $K \times 1$ vector on the left hand side of (27) by $Y_{\text {ajmt }}$. Stack equation (27) for all feasible combinations of actions $(a, j) \in \mathcal{A}$ to obtain the following equation

$$
Y_{m t}=\widetilde{X}_{m t} \theta+u_{m t}
$$

where $Y_{m t}, \tilde{X}_{m t}$, and $u_{m t}$ stack the vectors $Y_{a j m t}, \tilde{X}_{a j m t}$ and $u_{\text {ajmt }}$, respectively. Let $Z_{m t}$ be an $L \times K$ matrix of instrumental variables with $L \geq P$. The parameter $\theta$ is identified provided

\footnotetext{
${ }^{22}$ Such identifying restrictions matter for some, but not all counterfactuals; see Aguirregabiria (2010), Norets and Tang (2014), Arcidiacono and Miller (2019a), and Kalouptsidi, Scott, and Souza-Rodrigues (2019) for more details.
} 
$E\left[Z_{m t} u_{m t}\right]=0$ and $\operatorname{rank}\left(E\left[Z_{m t} \widetilde{X}_{m t}\right]\right)=P$.

Compared to Proposition 1, Proposition 2 imposes a linear-in-parameter payoff function, but does not require the single-action finite dependence property for some $J$, nor pre-specifying the payoff of that same action $J$. General patterns of finite dependence, including exchangeable sequences, can be accommodated because the regression function in (31) is a linear function of the parameters $\theta$ for any such sequences of choices. Proposition 2 can therefore be used to extend previous empirical applications exploring more than one-period (and not just single-action) finite dependence to incorporate serially correlated unobservable states and endogeneity (e.g., Coate, 2016; Matsumoto, 2016; Ma, 2019). The extension of Proposition 2 to stochastic finite dependence is shown in the Online Appendix (see Proposition ?? in Online Appendix ??).

It is important to notice that while Proposition 2 does not require the payoff parameters of a particular action to be known, the rank condition $\operatorname{rank}\left(E\left[Z_{m t} \widetilde{X}_{m t}\right]\right)=P$ still implicitly limits the number of parameters than can be identified. See, e.g., the dynamic land use change model discussed in Example 2. However, the condition is straightforward to check for a given data set and parametric specification.

\subsection{Discussion: Instrument Validity}

In this subsection, we discuss conditions for instrument validity. We consider the two components

of the econometric error term, $u_{m t}=\widetilde{\xi}_{m t}+\widetilde{e}_{m t}^{V}$. We start the discussion with the structural unobservable term $\widetilde{\xi}_{m t}$, and then we move to the expectational error $\widetilde{e}_{m t}^{V}$. To simplify, we ignore endogeneity issues related to one component when discussing the other one, but one should keep in mind that instrumental variables must be uncorrelated with both components. We close this subsection by focusing on the specifics of our four examples.

Structural Errors $\xi$. Recall that $\xi_{m t}$ is a function of observed and unobserved market-level states $\omega_{m t}=\left(w_{m t}, \eta_{m t}\right)$. If (a) $\xi_{m t}$ is a function of $\eta_{m t}$ but not of $w_{m t}$, and (b) the observed and unobserved market-level states, $w_{m t}$ and $\eta_{m t}$, evolve independently to each other, then $w_{m t}$ and $\xi_{m t}$ are fully independent and $w_{m t}$ can be taken as exogenous in the regression equation (27). In contrast, if $\xi_{m t}$ depends on $w_{m t}$ (e.g., when $\xi$ captures measurement errors), or if $w_{m t}$ and $\eta_{m t}$ do not evolve independently (e.g., when $w$ are observed prices and $\eta$ captures quality shocks in demand models), then $w_{m t}$ and $\xi_{m t}$ are correlated, in which case the use of excluded instruments becomes necessary for identification.

One possible source of instruments comes from the structure of simultaneity problems, as the identification of demand models using supply shifters as instruments; see, e.g., De Groote and Verboven (2019). Another possibility is to use Hausman-type of instruments by exploring independence across markets $m$ and using observable states in other markets, as we do in our taxi drivers' empirical illustration. 
A third possible source of instruments in panel data settings is lagged regressors. Requiring lagged state variables to be uncorrelated with $\widetilde{\xi}_{m t}$ demands restrictions on the evolution of $\xi_{m t}$. For instance, suppose $w_{m t}$ is serially correlated but $\widetilde{\xi}_{m t}$ is not, as when $\widetilde{\xi}_{m t}=\xi_{m t}$ captures i.i.d. measurement error in $w$. Then, any lagged regressor, $w_{m t-d}$ with $d \geq 1$, may serve as valid instrument. Lagged covariates may also serve as instruments when $\widetilde{\xi}_{m t}=\xi_{m t}$, but $\xi_{m t}$ is itself serially correlated (as in Example 1; see the Monte Carlo study in Section 6). More generally, when $\widetilde{\xi}_{m t}$ incorporates both $\xi_{m t}$ and future values $\xi_{m t+1}$ (as in Examples 2 and 3 above), then $\widetilde{\xi}_{m t}$ will be serially correlated by construction (even when $\xi_{m t}$ is i.i.d.), which means that further lags of $w_{m t}$ may be needed to obtain valid instruments. ${ }^{23}$

The use of lagged states as instrumental variables in dynamic models is also explored by Berry and Compiani (2019). Similar to the ECCP approach, they allow for serially correlated unobservable states that also correlate with observable states, resulting in econometric endogeneity problems. However, in contrast to the ECCP approach, they assume that the unobservable states are correlated with the controlled states, but are fully independent of the uncontrolled states. In our notation, they assume that $\xi$ (or $\eta$ ) correlate with $k$, but $\xi$ (or $\eta$ ) evolves independently of $w$, rendering lagged $w$ valid instruments for $k$. The ECCP approach addresses a different endogeneity problem: it does not require full independence between $\xi$ and $w$, but it does require that the transition process for $k$ does not depend on unobserved states. ${ }^{24}$

Expectational Errors $e^{V}$. We now discuss the expectational error term, $\widetilde{e}_{m t}^{V}$. It is clear that, to obtain variables that are uncorrelated with prediction errors, we need to make (minimal) assumptions on agents' information sets and beliefs. One common assumption in the applied literature is rational expectations.

Assumption 4. (Rational expectations) Agent's expectations conditional on the information set $\mathcal{I}_{\text {imt }}$ correspond to the conditional expectations of the true data generating process given $\mathcal{I}_{\text {imt }}$.

Rational expectations is useful for the ECCP approach because it offers sources of instrumental variables. Intuitively, when agents have rational expectations, forecast errors are mean independent of variables that belong to the agent's information set $\mathcal{I}_{\text {imt }}$ (Hansen and Sargent, 1980; Hansen and Singleton, 1982). The next lemma formalizes this intuition in the present context:

Lemma 1. Suppose Assumption 4 holds. Then,

\footnotetext{
${ }^{23}$ The unobservable $\widetilde{\xi}_{m t}$ may also correlate over time when it involves an additive market-level fixed-effect. In this case, it is possible to difference the fixed-effect out as usually done in linear panel data settings.

${ }^{24}$ Berry and Compiani (2019) estimate the policy function in the first step by regressing actions on states, and then estimate model parameters in the second step in a spirit similar to Bajari, Benkard, and Levin (2007). They make use of the generalized instrumental variable (GIV) approach proposed by Chesher and Rosen (2017) in the first step. When actions are discrete, the policy function is partially identified, implying set-identified model parameters in the second step. Importantly from a practical point of view, while the GIV approach allows for a large number of unobservables in theory, it is computationally difficult to calculate the identified sets in the presence of many unobservables. The ECCP approach does not suffer from this difficulty.
} 
(i) For any action $a$ and individual state $k$, the expectational error term $e^{h}\left(a, k, \omega_{m t}, \omega_{m t+1}^{*}\right)$ is mean zero given the information set available to the agent: $E\left[e^{h}\left(a, k, \omega_{m t}, \omega_{m t+1}^{*}\right) \mid \mathcal{I}_{i m t}\right]=0$.

(ii) For $z_{m t} \in \mathcal{I}_{\text {imt }}, E\left[e^{h}\left(a, k, \omega_{m t}, \omega_{m t+1}^{*}\right) \mid z_{m t}\right]=0$, for all $a$ and $k$.

(iii) Expectational errors are serially uncorrelated.

Lemma 1 shows that, when agents have rational expectations, and when instruments belong to agents' information sets, expectational errors $e^{V}$ are mean independent of $z_{m t}$. Assumption 4 therefore implies Assumption 3.(iii). ${ }^{25}$ Furthermore, because lagged values of $w_{m t}$ are in agent's contemporaneous information set, they can serve as instruments under the rational expectations assumption.

Future values of the state variables, in contrast, cannot be used as instruments because they correlate with forecast errors. Specifically, recall that the expectational error term at $t$ captures the difference between realized value functions at $t+1, V_{m t+1}$, and their time- $t$ expectations, $E\left[V_{m t+1} \mid \mathcal{I}_{i m t}\right]$, and note that the realization of the value function at $t+1$ will be correlated with realized covariates at $t+1, w_{m t+1}$. Put differently, $w_{m t}$ is not strictly exogenous in the regression equation (27), making lead covariates invalid instruments.

Another important and related aspect of the problem is that in some (but not all) models, the regression function in (27) includes future values of the covariates. In parametric models, the right-hand-side variable $\widetilde{X}_{m t}$ in equation (31) may include covariates observed at $t+1$ or subsequent periods, as specified in (30) (see, e.g., Example 3). As explained in the previous paragraph, future values of $w_{m t}$ correlate with forecast errors; this creates a mechanical endogeneity problem in the ECCP equation. For such cases, the researcher needs appropriate instruments in the contemporaneous information set for those future values of covariates. ${ }^{26}$

To be more concrete, we now close this section by focusing the discussion of instrument validity exclusively in the context of our examples. In the durable demand model (Example 1), the econometric error term is $u_{m t}=\xi_{m t}+\widetilde{e}_{m t}^{V}$, with $\xi_{m t}$ capturing serially correlated unobserved product quality shocks. In our Monte Carlo excercise (Section 6), we use supply shifters as instruments for product prices, assuming supply shifters are uncorrelated with demand shocks $\xi$, and assuming

\footnotetext{
${ }^{25}$ Strictly speaking, Lemma 1 implies that $E\left[e_{a j m t}^{V} \mid z_{m t}\right]=0$ while condition (iii) of Assumption 3 requires $E\left[\widetilde{e}_{a j m t}^{V} \mid z_{m t}\right]=0$, where $\widetilde{e}_{a j m t}^{V}$ includes $e_{a j m t}^{V}$ as well as potentially $e_{a j m, t+d}^{V}$ terms with $d \geq 1$. As discussed in the proof of Lemma 1 , an instrument $z_{m t}$ in the time- $t$ information set will also be uncorrelated with future expectational error terms.

${ }^{26}$ Endogeneity problems due to expectational errors are also a central concern in Dickstein and Morales (2018) and Morales, Sheu, and Zahler (2019). They construct discrete-choice analogues of Euler equations by comparing observed choice paths and paths involving one-period deviation in models with one-period finite dependence. Given that observed paths should be preferred to deviations, revealed-preference arguments provide the basis to construct moment inequalities, featuring forecast errors at the $t+1$ time period. While their approach allows for large choice sets (which is difficult to accomodate in the ECCP approach), it rules out unobserved heterogeneity, including serially correlated unobserved states, implying that observationally equivalent agents always prefer the same path of choices than the constructed alternative deviations.
} 
consumers do not make systematic mistakes in predicting future values $V_{m t}$, so that consumers' forecast errors $\widetilde{e}^{V}$ do not correlate with supply shocks. In the dynamic land use change model (Example 2), Scott (2013) uses lagged observed returns as instruments for contemporaneous returns, $R$ (crops, $\left.w_{m t}\right)-R\left(\right.$ other,$\left.w_{m t}\right)$, therefore imposing restrictions on the serial correlation of the econometric error term. Scott also assumes farmers have rational expectations, so that lagged returns do not correlate with expectational errors by construction. ${ }^{27}$ In the technology adoption problem (Example 3), the econometric error term includes both present and future demand shocks, $\xi_{m t}$ and $\xi_{m t+1}$, in addition to the prediction error. De Groote and Verboven (2019) use time-t cost shifters as instruments for PV prices (as mentioned previously), assume these shifters are part of households' information sets, and impose rational expectations. Under these assumptions, cost shifters are uncorrelated with both demand shocks and forecast errors. In our taxi drivers' labor supply application (Example 4), we take each working day as a market and use hourly earnings in previous days (i.e., in other markets) as instruments for today's earnings. We assume unobservable factors are independent across markets, and assume rational expectations (so that earnings in previous days are part of agents' information sets).

\subsection{Extensions}

We now briefly discuss three relevant extensions to the ECCP framework: the potential use of quasi-experiments as plausible sources of exogenous variation, models with biased beliefs or perfect foresight as alternatives to the standard rational expectations framework, and models with learning.

Quasi-Experiments. The ECCP approach can be combined with randomized control trials or quasi-experiments to identify the model parameters. For instance, Diamond, McQuade, and Qian (2018) extend the linear IV approach to a set of difference-in-differences regressions that explore variation in the assignment of rent control due to a 1994 ballot initiative in San Francisco. The idea is that equation (27) holds for both (randomly assigned) treatment and control groups. Given that families in both groups can live in the same neighborhood $m$, it is possible to difference (27) across groups to eliminate local unobservable amenities $\xi_{m t}$ that correlate with observable variables $w_{m t}$ and estimate the model parameters. In this way, they avoid the need to find instrumental variables to handle the correlation between $\xi$ and $w$.

Biased Beliefs and Perfect Foresight. Although rational expectations is a useful assumption that helps find plausible instrumental variables, it is clear that Propositions 1 and 2 do not require such assumption. Identification of model primitives can therefore be obtained under biased beliefs. The challenge in this case is to find plausible instruments that do not correlate with systematic errors $e^{V}$.

\footnotetext{
${ }^{27}$ In addition, Scott (2013) differences out county-level fixed-effects in the ECCP regression.
} 
The main difficulty under biased beliefs is not just that expectational errors are not mean zero, it is that the errors may also be serially correlated. Given that systematic prediction errors correlate with serially correlated covariates $w_{m t}$, they then also correlate with lagged covariates $w_{m t-d}$, turning lagged regressors invalid instruments. Unless the researcher imposes more restrictions on agents' beliefs (such as imposing some learning process combined with limited memory so that systematic mistakes do not extend for more than a limited number of time periods), the researcher cannot make use of lagged regressors as instrumental variables.

An alternative is to construct other moment restrictions based on (27) or (30) that do not require rational expectations. That is indeed the solution proposed by Diamond, McQuade, and Qian (2018). While they exploit the difference in treatment and control groups to eliminate local unobserved amenities $\xi_{m t}$, as mentioned previously, they still need to handle endogeneity problems related to the expectational error terms - composed of the difference between the forecast errors of treatment and control groups. In their differenced Euler equations, there is a mechanical correlation betweeen future covariates in the regression function and forecast errors. They use lagged states in agents' information sets as instruments. While lagged states may correlate with forecast errors under biased beliefs, they assume that the difference in expectations between treatment and control households in the same year are zero on average. I.e., agents may have biased beliefs, but cannot have beliefs that differ systematically across groups. That is a plausible assumption given the random assignment of households to treatment and control groups. They therefore combine their difference-in-differences approach with instrumental variables to estimate their model.

At the other extreme, the same Euler equation (27) can be derived from the assumption that agents have perfect foresight about the market state $\omega_{m t}$. Recall that our regression equations have residuals of the form $u_{m t}=\widetilde{\xi}_{m t}+\widetilde{e}_{m t}^{V}$. When agents have perfect foresight about $\omega_{m t}$, the unobservable component of the payoff becomes the entire residual, i.e., $u_{m t}=\widetilde{\xi}_{m t}$. This reinterpretation of the residual is of no consequence for the method-of-moments estimators we propose, since the moments depend only on the value of the entire residual $u_{m t}{ }^{28}$

Learning Models. The ECCP approach can be used to study learning models in which learning depends on individuals' choices and experiences. For Bayesian learning processes, the same number (and intensity) of signals along two exchangeable sequences of choices result in the same expected distribution of a future period's beliefs. I.e., the timing of the signals (obtained from individual choices) that reveal information to agents about an underlying parameter does not matter, while the number of signals do; this is a mechanical result from Bayesian updating. In this way, one can take advantage of exchangeable finite dependence to study decisions under information frictions (see, e.g., Arcidiacono, Aucejo, Maurel, and Ransom, 2016; Matsumoto, 2016). Similarly, learning-

\footnotetext{
${ }^{28}$ Note however that a misspecified perfect foresight model would ignore the mechanical endogeneity problem coming from the expectational error terms (as discussed above, expectational error terms are generally correlated with realized values of future variables). This would give the wrong impression that future covariates could be used as instruments, which in turn would produce biased parameter estimates.
} 
by-doing models with (stochastic) organizational forgetting, as in Besanko, Doraszelski, Kryukov, and Satterthwaite (2010), can exploit exchangeable finite dependence to identify and estimate the model primitives. For both types of learning models, the ECCP approach can allow for unobservable states and be flexible with respect to how these states evolve over time.

\section{Two-stage Estimation}

In this section, we present the ECCP estimator and establish its asymptotic properties. We follow the tradition of Hotz and Miller (1993) and estimate conditional choice probabilities and transition probabilities in the first step, and estimate the model parameters in the second step. To simplify exposition, we assume a large number of individuals $N$ and markets $M$, but we hold the number of time periods $T$ fixed. Asymptotic results can be extended to large $T$ by imposing stationarity and ergodicity assumptions.

Although it is possible to nonparametrically estimate payoff functions $\bar{\pi}$ (following Proposition 1 ), here we consider a parametric model $\bar{\pi}\left(a, k, w ; \theta_{0}\right)$, where $\theta_{0} \in \Theta \subset \mathbb{R}^{P}$ is the parameter of interest. Parametric models estimated using panel data involving large number of cross-sectional observations and small number of time periods is common in applied work. ${ }^{29}$

\section{$5.1 \quad$ First Stage}

In the first stage, we estimate $p_{a m t}($.$) and F_{a m t}^{k}($.$) in all markets m$ and all available time periods $t$. Denote the estimators for the CCPs and transition probabilities by $\widehat{p}_{\text {amt }}$ and $\widehat{F}_{a m t}^{k}$. Because $K$ is finite, we consider the frequency estimators: ${ }^{30}$

$$
\begin{aligned}
\widehat{p}_{a m t}(k) & =\frac{\sum_{i=1}^{N} 1\left\{a_{i m t}=a, k_{i m t}=k\right\}}{\sum_{i=1}^{N} 1\left\{k_{i m t}=k\right\}}, \\
\widehat{F}_{a m t}^{k}\left(k^{\prime} \mid k\right) & =\frac{\sum_{i=1}^{N} 1\left\{a_{i m t}=a, k_{i m t}=k, k_{i m t+1}=k^{\prime}\right\}}{\sum_{i=1}^{N} 1\left\{a_{i m t}=a, k_{i m t}=k\right\}} .
\end{aligned}
$$

We impose the following condition for each market $m$ and each time period $t$.

\footnotetext{
${ }^{29}$ Nonparametric payoff functions $\bar{\pi}$ can be estimated in the second step using estimators proposed by Newey and Powell (2003) or by Escanciano, Hoderlein, Lewbel, Linton, and Srisuma (2018). While the former involves ill-posed inverse issues, the latter combines standard kernel estimation with the computation of a matrix eigenvector problem in a way that avoids the ill-posed inverse problem. Aradillas-Lopez (2015) investigates the semiparametric efficiency properties of estimators of parametric models with rational expectations in which agents' beliefs are treated nonparametrically as "generated regressors." This approach may be useful in our context when $k$ is a continuous variable.

${ }^{30}$ Recall that results can be adapted to more complicated estimators of CCP and transitions. For instance, we can incorporate time-invariant observables that affect individuals' choices, as well as unobservable heterogeneity as in Kasahara and Shimotsu (2009) and Arcidiacono and Miller (2011) (see also Scott, 2013).
} 
Condition 1. The observations $\left\{a_{i m t}, k_{i m t}: i=1, \ldots, N\right\}$ are i.i.d. conditional on the market level state $\omega_{m t}$.

Condition 1 formalizes the idea that $\omega_{m t}$ is a common shock affecting all agents $i$ in market $m$ at time period $t$. As shown in Andrews (2005), this assumption is valid when the sample of individuals are drawn randomly from the population. (Sources of spatial dependence among agents within markets other than the common shock $\omega$ can be accommodated in our framework, but is beyond the scope of the paper.)

The probability limits of $\widehat{p}_{a m t}$ and $\widehat{F}_{a m t}$ can be determined following the Law of Large Numbers for exchangeable random variables (see Hall and Heyde, 1980). In addition, the result can be strengthened to a law of iterated logarithm, which is an important input to derive the asymptotic results for the second step. To simplify notation, stack the vectors $\left(\widehat{p}_{a m t}, \widehat{F}_{a m t}^{k}\right)$ and $\left(p_{a m t}, F_{a m t}^{k}\right)$ for all actions and states, and denote them, respectively, by $\widehat{\delta}_{m t}$ and $\delta_{m t}$. Note that $\delta_{m t}$ is itself random because $\left(p_{a m t}, F_{a m t}^{k}\right)$ depends on the realization of $\omega_{m t}$. In what follows, we use the Euclidean norm $\|\cdot\| \cdot{ }^{31}$

Lemma 2. Suppose Condition 1 holds. Then,

$$
\widehat{\delta}_{m t} \rightarrow \delta_{m t} \text { a.s. }
$$

as $N \rightarrow \infty$. Moreover,

$$
\left\|\widehat{\delta}_{m t}-\delta_{m t}\right\|=O_{a . s .}\left(\sqrt{\frac{\log \log N}{N}}\right) .
$$

\subsection{Second Stage}

Recall that, for each combination of $a$ and $j$, the unobservable $u_{\text {ajmt }}$ is the $K \times 1$ vector satisfying (27). Define the vector $u_{m t}\left(\theta, \delta_{m t}\right)$ that stacks $u_{a j m t}$ for all feasible combinations of $(a, j)$, and define the function $g_{m t}(\theta)=h\left(z_{m t}\right) u_{m t}\left(\theta, \delta_{m t}\right)$, where $h\left(z_{m t}\right)$ is a conformable function of the instrumental variables. The unconditional moment restriction (implied by Assumption 3) is then

$$
E\left[h\left(z_{m t}\right) u_{m t}\left(\theta_{0}, \delta_{m t}\right)\right]=0
$$

Define the function $g(\theta) \equiv E\left[g_{m t}(\theta)\right]$; the GMM population criterion function is given by

$$
Q(\theta)=g(\theta)^{\prime} \mathbf{W} g(\theta)
$$

\footnotetext{
${ }^{31}$ Although we do not exploit the asymptotic distribution of $\widehat{\delta}_{m t}$ in this paper, note that it can be obtained following the arguments in Andrews (2005). Specifically, under the regularity conditions stated in Andrews (2005), $\sqrt{N}\left(\widehat{\delta}_{m t}-\delta_{m t}\right)$ converges in distribution to a mixture of normal distributions that depend on the common shock $\omega_{m t}$.
} 
where $\mathbf{W}$ is a (non-stochastic) positive-definite weighting matrix. By the identification results, $\theta_{0}$ is the unique minimizer of $Q(\theta)$.

Next, consider the sample analogue of (35). Define the functions

$$
\begin{aligned}
\widehat{g}_{m t}(\theta) & =h\left(z_{m t}\right) u_{m t}\left(\theta, \widehat{\delta}_{m t}\right), \text { and } \\
\widehat{g}_{M}(\theta) & =\frac{1}{M(T-\tau)} \sum_{m=1, t=1}^{M,(T-\tau)} \widehat{g}_{m t}(\theta),
\end{aligned}
$$

where $\tau$ reflects the $\tau$-finite dependence assumption (see Definition 2). The GMM criterion function is then given by

$$
\widehat{Q}_{M}(\theta)=\widehat{g}_{M}(\theta)^{\prime} \mathbf{W}_{M} \widehat{g}_{M}(\theta)
$$

where $\mathbf{W}_{M}$ is a positive-definite weighting matrix that may depend on data. The estimator $\widehat{\theta}_{M}$ minimizes $\widehat{Q}_{M}(\theta)$ over $\Theta$.

The next set of conditions suffices for consistency of $\widehat{\theta}_{M}$.

Condition 2. Conditions for consistency:

(i) The vector $\left(w_{m t}, z_{m t}\right)$ is i.i.d. across markets $m$.

(ii) $\mathbf{W}_{M} \stackrel{p}{\rightarrow} \mathbf{W}$ as $M \rightarrow \infty$.

(iii) $\Theta$ is compact.

(iv) $\theta_{0}$ uniquely minimizes $Q(\theta)$ over $\Theta$.

(v) $\bar{\pi}_{\text {amt }}(\theta)$ is continuous at each $\theta \in \Theta$ for all $a \in \mathcal{A}$.

(vi) $E\left[\sup _{\theta \in \Theta}\left\|h\left(z_{m t}\right) u_{m t}\left(\theta, \delta_{m t}\right)\right\|\right]<\infty$.

(vii) $E\left[\sup _{\theta \in \Theta}\left\|h\left(z_{m t}\right) \nabla_{\delta} u_{m t}\left(\theta, \delta_{m t}\right)\right\|^{2}\right] \leq B<\infty$, where $B$ is a finite constant.

Condition 2 establishes standard regularity conditions that guarantee the problem is wellbehaved. The assumption that market-level variables are independent across markets simplifies the derivation of the asymptotic results (Condition 2(i)), but results can be extended to allow for spatial dependence across markets (Conley, 1999; Andrews, 2005; Kuersteiner and Prucha, 2013). Condition 2(ii)-(vi) are standard. Condition 2(vii) is used in conjunction with equation (32) in Lemma 2 of Section 5.1, to guarantee uniform convergence in probability of the criterion function to its population version. The term $\nabla_{\delta} u_{m t}$ is the derivative of the vector $u_{m t}$ with respect to $\delta$, which in turn, depends on the derivative of $\psi_{a}$ with respect to the CCPs (see equation (27)). As shown in Kalouptsidi, Scott, and Souza-Rodrigues (2019), $\psi_{a}$ is indeed a differentiable function of $p(k, \omega)$, provided $p(k, \omega)$ lies strictly between zero and one (which is satisfied when payoffs are bounded and $\varepsilon_{\text {imt }}$ has full support on $\mathbb{R}^{A+1}$ ).

Proposition 3. Under Conditions 1 and $2, \widehat{\theta}_{M} \stackrel{p}{\rightarrow} \theta_{0}$ as $(M, N) \rightarrow \infty$.

To obtain the asymptotic distribution of $\widehat{\theta}_{M}$, we impose the following: 
Condition 3. Conditions for asymptotic distribution:

(i) $\theta_{0} \in$ interior $(\Theta)$.

(ii) $\bar{\pi}_{a m t}(\theta)$ is continuously differentiable in a neighborhood $\mathcal{N}$ of $\theta_{0}$ with probability approaching one for all $a \in \mathcal{A}$.

(iii) $E\left[\left\|h\left(z_{m t}\right) u_{m t}\left(\theta_{0}, \delta_{m t}\right)\right\|^{2}\right]<\infty$, and $E\left[\sup _{\theta \in \mathcal{N}}\left\|h\left(z_{m t}\right) \nabla_{\theta} u_{m t}\left(\theta, \delta_{m t}\right)\right\|\right]<\infty$.

(iv) $\mathbf{G}^{\prime} \mathbf{W G}$ is nonsingular for $\mathbf{G}=E\left[\frac{1}{T-\tau} \sum_{t=1}^{T-\tau} h\left(z_{m t}\right) \nabla_{\theta} u_{m t}\left(\theta_{0}, \delta_{m t}\right)\right]$.

Similar to Condition 2, Condition 3 imposes standard regularity conditions to make the problem well-behaved. The next proposition follows.

Proposition 4. Suppose Conditions 1, 2 and 3 hold. Assume $(M \log \log N) / N \rightarrow 0$, as $(M, N) \rightarrow$ $\infty$. Then,

$$
\sqrt{M}\left(\widehat{\theta}_{M}-\theta_{0}\right) \stackrel{p}{\rightarrow} N(0, \mathbf{V})
$$

where

$$
\mathbf{V}=\left(\mathbf{G}^{\prime} \mathbf{W} \mathbf{G}\right)^{-1} \mathbf{G}^{\prime} \mathbf{W} \Sigma \mathbf{W G}\left(\mathbf{G}^{\prime} \mathbf{W} \mathbf{G}\right)^{-1}
$$

and

$$
\Sigma=E\left[\left(\frac{1}{T-\tau} \sum_{t=1}^{T-\tau} g_{m t}\left(\theta_{0}\right)\right)\left(\frac{1}{T-\tau} \sum_{t=1}^{T-\tau} g_{m t}\left(\theta_{0}\right)\right)^{\prime}\right]
$$

The asymptotic distribution of $\widehat{\theta}_{M}$ in Proposition 4 is the same as the distribution of an unfeasible estimator in which $\delta_{m t}$ is observed instead of estimated in the first step. The first step estimators $\widehat{\delta}_{m t}$, for all markets and time periods, do not affect the asymptotic variance of $\widehat{\theta}_{M}$ when the number of observations within markets $N$ is sufficiently large compared to the number of markets $M$. The rate at which $N$ must increase to eliminate the influence of the first step on the variance of the second step is $\frac{M \log \log N}{N} \rightarrow 0$ as $(M, N) \rightarrow \infty$. This rate is a direct result of the Law of Iterated Logarithm obtained in Lemma $2 .^{32}$

Consistent estimators for $\mathbf{V}$ and optimal weighting matrices for $\mathbf{W}$ can be obtained using standard arguments and are omitted here (see, e.g., Theorem 4.5 in Newey and McFadden (1994)).

We close the section with a discussion of two practical concerns regarding asymptotic inference.

Remark 4. (Macroeconomic Shocks.) The presence of aggregate shocks can pose difficulties to estimation and inference in nonlinear panel data models in general (see, e.g., Hahn, Kuersteiner, and Mazzocco, 2019). In the present case, the difficulty may depend on whether the researcher has a short or long panel. Recall that when the instruments belong to the agent's contemporaneous information set, and when agents have rational expectations, the instruments are uncorrelated with $\widetilde{e}_{m t}^{V}$. Yet, it is important to note that, while the expectational error terms are mean-zero given $\mathcal{I}_{i m t}$, there is a distinction between the way $e^{V}$ averages out in the cross-sectional dimension $M$ and the

\footnotetext{
${ }^{32}$ When $N$ is not sufficiently large relative to $M$, the first step may affect the distribution of the estimator in the second step. See Chernozhukov, Escanciano, Ichimura, Newey, and Robins (2018) and Dearing (2019) for strategies to orthogonalize the influence of the first step on the distribution of $\widehat{\theta}_{M}$.
} 
way it averages out in the time-series dimension T. For instance, if in a given time period $t$, a macro shock affects all agents in all markets, then the prediction errors $e^{V}$ do not average out to zero asymptotically when $M \rightarrow \infty$. For this averaging out to happen as the cross-section becomes large, we do need to make a substantive assumption about the correlation across markets. Such type of assumption may be appropriate for some applications, but it may not be appropriate when, for example, markets are geographically defined and state variables include prices whose movements over time are strongly correlated around the world (e.g., transportable commodities).

In contrast, in a large-T setting, asymptotic convergence involving $e^{V}$ terms does not require a substantive assumption about how markets are correlated. Instead, rational expectations guarantees that expectational error terms are serially uncorrelated (see Lemma 1). Intuitively, when $T \rightarrow \infty$, macro shocks wash out in the limit. We investigate this possibility in our Monte Carlo exercise by exploring the finite sample performance of the ECCP estimator with different sample sizes $M$ and $T$, both in the presence and absence of macro shocks.

Remark 5. (Observation Weights.) The efficiency of the ECCP approach can potentially be improved by considering observation weights in the second stage. In general, in the presence of heteroskcedastic errors, optimal regression weights are inversely proportional to the variance of each observation's error term $u_{m t}$. The variance of the error term depends on the (first-stage) choice probabilities and can therefore be inferred from the choice probability estimates and the sample size used to estimate them. From this, the variance of the dependent variable in equation (16) can be computed, yielding appropriate observation weights. Note that these weights can reduce the influence of imprecisely estimated first stage CCP's, since, in practice, even large data sets can have small sample sizes after conditioning on certain state variables.

\section{Monte Carlo}

In this section, we present a Monte Carlo experiment to illustrate the performance of the ECCP estimator, focusing on the dynamic demand model for a durable good discussed in Example 1.

Recall that the choice set is $\mathcal{A}=\{b, n b\}$, where $a=b$ if the consumer buys the good, and $a=n b$ if she does not buy it. The individual-level state $k_{i m t}$ reflects whether she already owns the product or not at the beginning of time period $t$. We consider two market-level state variables: observed price $w_{m t}$ and unobservable quality $\xi_{m t} \cdot{ }^{33}$ We also consider an observed supply (cost) shifter $z_{m t}$ (that will play the role of an instrumental variable). The price is a function of $z_{m t}$ and $\xi_{m t}$, determined as follows:

$$
w_{m t}=\gamma_{0}+\gamma_{1} z_{m t}+\gamma_{2} \xi_{m t}+\varepsilon_{m t}^{w}
$$

\footnotetext{
${ }^{33}$ Recall that, formally, we can define $\eta_{m t}$ as the state variable, while the function $\xi\left(a, k, \omega_{m t}\right)$ equals $\eta_{m t}$ when $a=b$, and equals zero when $a=n b$. I.e., the unobserved quality enters into the utility of purchasing the good and not into the utility of not purchasing (whether the good is already owned or not). To simplify notation, we take $\xi_{m t}$ as the state variable.
} 
where $\varepsilon_{m t}^{w}$ is a mean-zero normally distributed i.i.d. price shock with variance $\sigma_{w}^{2}$. Note that $\gamma_{1}$ represents how variation in the observed cost shifter $z_{m t}$ passes through to prices. $\xi_{m t}$ is included in the price equation to capture the idea that demand shocks may influence the price.

The supply shifter $z_{m t}$ and unobserved quality $\xi_{m t}$ follow independent $\operatorname{AR}(1)$ processes:

$$
\begin{aligned}
& \xi_{m t+1}=\rho_{1}+\rho_{2} \xi_{m t}+\varepsilon_{m t}^{\xi}, \\
& z_{m t+1}=\rho_{3}+\rho_{4} z_{m t}+\varepsilon_{m t}^{z},
\end{aligned}
$$

where $\varepsilon_{m t}^{\xi}$ and $\varepsilon_{m t}^{z}$ are normally distributed zero-mean i.i.d. shocks with variances $\sigma_{\xi}^{2}$ and $\sigma_{z}^{2}$, respectively. Recall that $\xi_{m t}$ has mean zero by assumption, so we take $\rho_{1}=0$.

We consider two settings in our simulations, depending on whether the unobservable state $\xi$ is present in the data generating process or not. When there is no unobserved states, we set $\xi_{m t}=0$ for all $m$ and $t$ (or, equivalently, we set $\sigma_{\xi}^{2}=0$ ).

As many applications may feature aggregate shocks, we also consider two scenarios: with and without macro shocks. In the scenario with aggregated shocks, we incorporate them into the term $\varepsilon_{m t}^{z}$. Specifically, we simulate $\varepsilon_{m t}^{z}=\varepsilon_{m, t, 1}^{z}+\varepsilon_{t, 2}^{z}$, where the (mean-zero) macro shock $\varepsilon_{t, 2}^{z}$ accounts for a fraction $\lambda_{z}$ of the variance in $\varepsilon_{m t}^{z}$. (Recall that macro shocks wash out in the limit when the number of time periods goes to infinity, but they may not wash out in the limit when the asymptotics is in the number of markets.)

We consider several possible sample sizes with different combinations of $M$ and $T$. For each sample structure, we generate 5,000 Monte Carlo replications. For the first sample period, we generate the value of the state variables from their steady-state distributions. The supports of the market-level state variables are discretized to take integer values. We solve the individual dynamic optimization problem by value function iteration on the discretized state space. ${ }^{34}$ The parameters of the data generating process in our Monte Carlo study is summarized in Table 1.

The main parameters of interest are the payoff parameters $\theta=\left(\theta_{0}, \theta_{1}\right)$, which are estimated based on the regression equation (21). We estimate $\theta$ using two ECCP estimators: the first estimator is based on the Ordinary Least Squares (OLS) estimator, while the second one is based on instrumental variables - specifically, the Two-Stage Least Squares (2SLS) estimator.

We also estimate $\theta$ using a standard CCP estimator similar to Hotz and Miller (1993). This procedure relies on a full specification of what the state variables are and how they evolve. To implement this procedure, we assume that the price $w_{m t}$ is the only market-level state variable and model its evolution as a first-order Markov process. So, when the unobserved quality is a relevant state (i.e., when $\sigma_{\xi}^{2}>0$ ), this strategy is based on a mis-specified model. See Online Appendix ?? for details.

\footnotetext{
${ }^{34}$ We simulate conditional choice probabilities for each market-period and assume these CCPs are observed by the econometrician. Thus, we abstract away from any first-stage sampling uncertainty in the estimation of choice probabilities and effectively assume a (sufficiently) large number of agents $N$ within each market-year.
} 
Table 2 presents results for a model with no unobservable demand shocks (i.e., $\sigma_{\xi}^{2}=0$ ). For each model parameter, each sample structure, and each estimator, we report the average estimate, the relative mean bias (as a percentage of the true parameter), the standard deviation, and the root-mean squared error (RMSE) of the estimator. On the left panel, we present results for the scenario with no macro shocks (i.e., $\lambda_{z}=0$ ), and on the right panel, we present the estimated results with aggregated shocks $\left(\lambda_{z} \neq 0\right)$.

In the absence of macro shocks, all estimation strategies appear to be consistent, as expected. Not surprisingly, the standard CCP approach exhibits smaller standard deviations and RMSE when compared to the ECCP OLS and IV estimators.

Table 2 also illustrates that there can be a difference between the number of markets and time periods for the asymptotic properties of ECCP estimators in the scenario with macro shocks $\left(\lambda_{z}=.7\right)$. We have several columns with the same sample size $M \times T=1600$, and within these columns, there is non-trivial bias in the OLS and IV estimates for short panels when the aggregate shocks are present. However, the bias is reduced when the time dimension increases, and for long panels, the ECCP estimators have little or no bias. ${ }^{35}$

Table 3 presents the results for the main specification, where the unobserved demand shock $\xi_{m t}$ is present. Because price $w_{m t}$ and the unobserved quality $\xi_{m t}$ are correlated by construction ( since $\gamma_{2} \neq 0$ ), we expect OLS to be biased. Indeed, the OLS estimator is now highly biased for both the intercept $\theta_{0}$ and price coefficient $\theta_{1}$.

The supply shifter $z_{m t}$ provides a valid instrument given that it correlates with prices $w_{m t}$ and is independent of $\xi_{m t}$, and mean-independent of the expectational errors. When using the cost shifter as an instrument for price, we see that the ECCP IV estimator has little-to-no bias, either in the absence of aggregate shocks or when $T$ is large (aggregate shocks still pose problems for short panels, as expected).

The standard CCP estimator is now severely biased, for it treats the market state space as including only the observable price $w_{m t}$ while the unobservable demand shock $\xi_{m t}$ also plays a role. The mis-specification is important regardless of the presence or absence of macro shocks. In particular, the relative bias of the standard CCP estimator is larger than the bias of the ECCP IV estimator in the presence of aggregated shocks in short panels.

Counterfactuals. While the ECCP approach avoids the need for a full model of how state variables evolve, counterfactual analysis typically requires a full model to solve and simulate a counterfactual dynamic problem. Thus, while the ECCP approach has an advantage in requiring weaker modeling assumptions for estimation, stronger assumptions are still needed to compute counterfactuals. Here we note that, when possible, it may be desirable to estimate model param-

\footnotetext{
${ }^{35}$ In our simulations, aggregate shocks only affect the evolution of the observable variables $\left(z_{m t}\right.$ directly, and $w_{m t}$ indirectly). However, these aggregate shocks in the observables lead to aggregate shocks in the expectational error terms. In turn, aggregate shocks in the expectational error terms implies that our regression equation's residual features aggregate shocks.
} 
eters with as few assumptions (and potential sources of bias) as possible, and impose additional assumptions needed for counterfactuals simulations only after estimating those parameters.

In the context of dynamic demand, the computation of a long-run elasticity represents a counterfactual of interest. Computing long-run elasticities requires an explicit model of how prices and demand shocks jointly evolve, even when ECCP estimation does not. We find that using the ECCP approach for estimation and then imposing restrictive assumptions on unobservable shocks only for counterfactuals outperforms an approach that imposes the same restrictive assumptions in both estimation and counterfactual simulation. ${ }^{36}$ Online Appendix ?? presents these results in detail.

\section{Empirical Application}

We now illustrate the ECCP approach in the context of an empirical labor supply model for taxi drivers in New York City (NYC) in 2013. ${ }^{37}$

As in many studies of labor supply, our goal is to estimate how labor supply responds to wages. The taxi driver data allows us to see how labor supply decisions respond to wages at the hourly level, but one might worry that the variation in taxi driver wages might be correlated with unobserved factors that shift taxi drivers' labor supply. Indeed, such correlations should be expected from the simultaneous determination of labor supply and wages. Fortunately, the ECCP framework allows us to easily incorporate instrumental variables in the estimation of a dynamic model of taxi driver labor supply.

Institutional Background. Operating a taxicab in NYC requires a medallion and a NYC Taxi and Limousine Commission (TLC) driver's license. Most drivers rent medallions from the owners or intermediaries and must return the car by the end of the shift (the daily lease rate in 2013 was between $\$ 115$ and $\$ 139)$. The cap for the total number of medallions was set at 13,437 in 2013. A common feature in NYC is the '2-shift rule': almost all rental agreements between drivers and taxi fleets start or end at $5 \mathrm{AM}$ or $5 \mathrm{PM}$; the day shift starts at $5 \mathrm{AM}$ and ends at $5 \mathrm{PM}$, while the night shift starts at 5 PM and ends at 5 AM. Fares are regulated by TLC and did not change during the sample period. For more details, see Schmidt (2019).

Data. The transaction-level data is based on the Taxicab Passenger Enhancement Project (TPEP), organized by TLC. The data contain information about every yellow cab trip, including drivers' identifier; information regarding the length, distance, and duration of the trip; the fare, surcharges, tolls, and tips paid by costumers; as well as the geo-spatial start and endpoint of the

\footnotetext{
${ }^{36}$ Specifically, we set the unobserved demand shock at its long-run mean when calculating the long-run demand elasticity. The unobserved demand shock has zero mean with no loss of generality, given that a non-zero mean term is absorbed by the constant term in the regression.

${ }^{37}$ We are grateful to Øystein Daljord and Walter Zhang, who generously shared the data with us.
} 
trip. We focus on data from January 1st to December 31st of 2013. We use a sample of 10,500 licensed drivers that have been active in that year. The transaction-level data is aggregated to the driver-hour level (indexed by $i$ and $t$, respectively), so that the unit of time $t$ is an hour of the day. Expected market hourly earnings $w_{m t}$ is defined as the total earnings (including fare and tips) averaged across drivers in that hour $t$ of that day $m$. We restrict our sample to consider only drivers who were working during the day shifts, and that worked for at least one hour and at most 12 hours. Following the literature, we also restrict the sample to the weekdays Monday-Thursday because supply patterns across these days are very similar (Buchholz, 2019; Fréchette, Lizzeri, and Review, 2019). The final data set used for estimation is an unbalanced panel with $N=3,937$ drivers observed in $M=209$ days during the $T=12$ hours between 5 AM and 5PM. ${ }^{38}$ The taxi data was merged to data on hourly weather conditions from Open Weather Map data and the National Weather Service (NWS) of the National Oceanic and Atmospheric Administration (NOA). These data contain information on temperature, atmospheric pressure and humidity, rainfall, and wind speed, among other weather conditions.

Table 4 shows the summary statistics by hour of the day, averaged across drivers and days. The table indicates that the share of drivers who decide to continue working in the morning hours is high (above 95\%). The share of working drivers then declines slowly in the afternoon, and drops substantially to $51 \%$ by 4 PM because they start to return to the garages before the end of shift by 5 PM. Average hourly earnings is $\$ 32$, with higher returns between 6 AM and 9 AM, when demand is higher. The average number of hours working is approximately 7.5 hours per day.

Model and Estimation. We consider the model presented in Example 4, extended to incorporate three additional types of observed market-level states: (i) dummy variables for important holidays (namely, Memorial Day, the Fourth of July, United Nations week, and New Year's Eve), (ii) dummy variables for weather conditions, as they may affect the disutility of driving, ${ }^{39}$ and (iii) the hour of the day $t$, which may reflect the fact that driving during some hours may be less pleasant (e.g., during lunch time or rush hours). We assume all these variables enter the flow payoffs additively. The discount factor is set at $\beta=0.999999$ (implying an annual interest rate of approximately $0.87 \%$ ).

To obtain the CCPs in the first stage, we estimate flexible logit regressions separately for each hour of the day. These regressions include a fourth-order polynomial on $k$ and dummies for the day of the month, the month in the year, the holidays, and the weather conditions - in doing so, we effectively smooth the CCPs across markets $m$ to improve accuracy given the sample size.

\footnotetext{
${ }^{38}$ Because the transaction-level data does not provide direct information of the contractual shifts, we follow the previous studies and define any time period of more than 6 hours between trips as time 'off work,' separating the time shifts. We consider day shifts starting any time between $5 \mathrm{AM}$ and $2 \mathrm{PM}$, and ending at any hour between 8 AM and 5 PM. To simplify, we ignore breaks during the shift and we do not model the spatial distribution of all trips - allowing for such extensions are possible, but beyond the scope of our paper.

${ }^{39}$ The weather dummies include the following classifications: Clear, Clouds, Drizzle, Fog, Haze, Mist, Rain, Snow, and Thunderstorm.
} 
The estimated results are not very sensitive to the parametric specification. ${ }^{40}$ In the second stage, we estimate equation (24) as explained in Example 4, and augmented to include the additional variables mentioned above.

Results. Table 5 presents the results. In the first column, we show the OLS estimates; in the second column, the 2SLS estimates using the within-day lagged earnings $\left(w_{m t-1}\right)$ as the instrumental variable for hourly earnings $\left(w_{m t}\right)$; and in the third column, the 2SLS estimates using the earnings in the same hour of the day but in the previous day $\left(w_{m-1, t}\right)$ as the instrument - a "Hausman-IV'. For ease of exposition, we omit from the table the coefficients on all state variables other than $w$ and $k$.

The estimated coefficients on hourly earnings $\left(\theta_{1}\right)$ are positive in all specifications, as expected, and the value of the OLS estimate is substantially smaller than the 2SLS estimates. This is consistent with the standard bias of OLS in demand and supply models: labor supply parameters are underestimated when simultaneity problems are ignored. Instrumenting for $w_{m t}$ increases the supply response to earnings. The 2SLS estimate for $\theta_{1}$ when using the within-day lagged earnings as the instrument is almost twice as large as the OLS estimate; and the 2SLS estimate based on the previous day earnings as the instrument is approximately six times larger than the OLS estimate (and it is statistically significant at the $5 \%$ level). Both instruments are strong, with the first-stage F-statistics well above usual critical values for weak-IV tests (Montiel Olea and Pflueger, 2013; Andrews, Stock, and Sun, 2019). Still, one might worry that the unobservables $\xi_{m t}$ might be highly persistent within the day, casting doubts on whether the within-day lagged earnings is a valid IV. (The fact that the first-stage F-statistic is extremely large for that instrument is consistent with that suspicion.) In that sense, our second IV (the previous day earnings) seems to be more plausibly exogenous. ${ }^{41}$

The estimated coefficients on the individual states $k\left(\theta_{2}\right)$ - corresponding to the fatigue cost of driving - are all negative as expected, and stable across specifications. Yet, the implied monetary values for the fatigue costs (i.e., the estimated ratio $\theta_{2} / \theta_{1}$ ) differ substantially: the implied value for the OLS estimates is approximately $\$ 81$ per hour of driving, which is much higher than the average hourly earnings: $\$ 32 /$ hour. The implied fatigue cost based on the within-day lagged IV estimates is smaller than the corresponding OLS costs but still greater than the average earnings: $\$ 43 /$ hour. Finally, the previous day IV results in estimates that are more plausible, around $\$ 13 /$ hour.

\footnotetext{
${ }^{40}$ We have also estimated flexible logits with polynomials of the 2 nd up to the 6 th order on $k$, and interaction terms between $k$ and the day of the month dummies, as well as between $k$ and the month of the year dummies, with qualitatively similar (but more noisy) results. The second stage estimates are not sensitive to changes in the definition of the shifts, to the inclusion of all days of the week, nor to the value of the discount factor $\beta$.

${ }^{41}$ We have also used the earnings in the same hour of the day and the same day of the week but in the previous week $\left(w_{m-7, t}\right)$ as the instrumental variable. This instrument is strong $(F=43.4)$ and delivers a similar point estimate as the "previous day" IV $\left(\hat{\theta}_{1}=0.0198\right)$, but the coefficient is less precisely estimated.
} 


\section{Conclusion}

In this paper we provide a comprehensive econometric treatment of a new class of linear instrumental variables estimators for structural dynamic discrete choice models: the ECCP estimators. This class of estimators shares many of the advantages of the continuous-choice Euler equation approach originally developed by Hall (1978), Hansen and Sargent (1980, 1982), and Hansen and Singleton (1982).

We provide constructive identification results that lead naturally to estimators, we establish the consistency and asymptotic normality of the estimators, we provide evidence that they perform well in finite-samples based on a Monte Carlo study of a dynamic demand for durable goods, and we illustrate the method empirically in the context of dynamic labor supply of taxi drivers in NYC.

\section{References}

Abbring, J. H., And O. Daljord (2019): "Identifying the Discount Factor in Dynamic Discrete Choice Models," Quantitative Economics, forthcoming.

Aguirregabiria, V. (2010): "Another look at the identification of dynamic discrete decision processes: An application to retirement behavior," Journal of Business $\&$ Economic Statistics, $28(2), 201-218$.

Aguirregabiria, V., J. Gu, and Y. Luo (2019): "Sufficient Statistics for Unobserved Heterogeneity in Structural Dynamic Logit Models," Journal of Econometrics, forthcoming.

Aguirregabiria, V., And A. Magesan (2013): "Euler equations for the estimation of dynamic discrete choice structural models," in Structural Econometric Models, pp. 3-44. Emerald Group Publishing Limited.

Aguirregabiria, V., And P. Mira (2002): "Swapping the Nested Fixed Point Algorithm: A Class of Estimators for Discrete Markov Decision Models," Econometrica, 70(4), 1519-1543.

Altug, S., And R. A. Miller (1998): "The Effect of Work Experience on Female Wages and Labour Supply," The Review of Economic Studies, 65(1), 45-85.

Andrews, D. W. (2005): "Cross-Section Regression with Common Shocks," Econometrica, $73(5), 1551-1585$.

Andrews, I., J. Stock, And L. Sun (2019): "Weak Instruments in IV Regression: Theory and Practice," Annual Review of Economics, 11, 727-753.

Aradillas-Lopez, A. (2015): "Semiparametric Efficiency in Models with Rational Expectations," Discussion paper, Pennsylvania State University. 
Arcidiacono, P., E. Aucejo, A. Maurel, and T. Ransom (2016): "College Attrition and the Dynamics of Information Revelation," Discussion Paper 22325, NBER.

Arcidiacono, P., And P. B. Ellickson (2011): "Practical Methods for Estimation of Dynamic Discrete Choice Models," Annual Review of Economics, 3(1), 363-394.

Arcidiacono, P., and R. A. Miller (2011): "Conditional Choice Probability Estimation of Dynamic Discrete Choice Models With Unobserved Heterogeneity," Econometrica, 79(6), 18231867.

(2019a): "Identifying Dynamic Discrete Choice Models off Short Panels," Journal of Econometrics, forthcoming.

- (2019b): "Nonstationary Dynamic Models with Finite Dependence," Quantitative Economics, 10, 853-890.

Bajari, P., C. L. Benkard, and J. Levin (2007): "Estimating Dynamic Models of Imperfect Competition," Econometrica, 75(5), 1331-1370.

Bajari, P., C. S. Chu, D. Nekipelov, and M. Park (2016): "Identification and semiparametric estimation of a finite horizon dynamic discrete choice model with a terminating action," Quantitative Marketing and Economics, 14(4), 271-323.

Benkard, C. L. (2000): "Learning and Forgetting: The Dynamics of Aircraft Production," American Economic Review, 90(4), 1034-1054.

Berry, S., And G. Compiani (2019): "An Instrumental Variables Approach to Dynamic Models," Discussion paper, Yale University.

Berry, S. T. (1994): "Estimating Discrete-Choice Models of Product Differentiation," RAND Journal of Economics, 25(2), 242-262.

Besanko, D., U. Doraszelski, Y. Kryukov, and M. Satterthwaite (2010): "Learningby-Doing, Organizational Forgetting, and Industry Dynamics," Econometrica, 78(2), 453-508.

Bishop, K. C. (2012): "A dynamic model of location choice and hedonic valuation," Discussion paper, Arizona State University.

Blevins, J. R. (2014): "Nonparametric identification of dynamic decision processes with discrete and continuous choices," Quantitative Economics, 5(3), 531-554.

Blevins, J. R., A. Khwaja, And N. Yang (2018): "Firm Expansion, Size Spillovers, and Market Dominance in Retail Chain Dynamics," Management Science, 64(9), 4070-4093. 
Buchrolz, N. (2019): "Spatial Equilibrium, Search Frictions and Dynamic Efficiency in the Taxi Industry," Discussion paper, Princeton University.

Chernozhukov, V., J. Escanciano, H. Ichimura, W. Newey, and J. Robins (2018): "Locally Robust Semiparametric Estimation," Discussion paper, arXiv:1608.00033v2.

Chernozhukov, V., And C. Hansen (2005): "An IV Model of Quantile Treatment Effects," Econometrica, 73(1), 245-261.

Chesher, A., And A. M. Rosen (2017): "Generalized Instrumental Variable Models," Econometrica, 85(3), 959-989.

Chiong, K. X., A. Galichon, and M. Shum (2016): "Duality in dynamic discrete-choice models," Quantitative Economics, 7(1), 83-115.

Chou, C., T. Derdenger, and V. Kumar (2019): "Linear Estimation of Aggregate Dynamic Discrete Demand for Durable Goods without the Curse of Dimensionality," Marketing Science, $38(5), 888-909$.

CoAte, P. (2016): "Parental influence on labor market outcomes and location decisions of young workers," Discussion paper, American Institute for Economic Research.

Conley, T. G. (1999): "GMM estimation with cross sectional dependence," Journal of Econometrics, 92(1), 1-45.

De Groote, O., and F. Verboven (2019): "Subsidies and Time Discounting in New Technology Adoption: Evidence from Solar Photovoltaic Systems," American Economic Review, 109(6), $2137-72$.

Dearing, A. (2019): "Pseudo-Value Functions and Closed-Form CCP Estimation of Dynamic Discrete Choice Models," Discussion paper, Ohio State University.

Diamond, R., T. McQuade, and F. Qian (2018): "The effects of rent control expansion on tenants, landlords, and inequality: Evidence from san francisco," Discussion paper, Stanford University.

Dickstein, M. J., And E. Morales (2018): "What Do Exporters Know?," The Quarterly Journal of Economics, 133(4), 1753-1801.

Escanciano, J. C., S. Hoderlein, A. Lewbel, O. Linton, and S. Srisuma (2018): "Nonparametric Euler Equation Identification and Estimation," Discussion paper, Boston College.

Fréchette, G. R., A. Lizzeri, and T. S. A. E. Review (2019): "Frictions in a Competitive, Regulated Market: Evidence from Taxis," American Eonomic Review, forthcoming. 
Gendron-Carrier, N. (2018): "Understanding the Careers of Young Entrepreneurs," Discussion paper, McGill University.

Gowrisankaran, G., and M. Rysman (2012): "Dynamics of consumer demand for new durable goods," Journal of Political Economy, 120(6), 1173-1219.

Hahn, J., G. Kuersteiner, And M. Mazzocco (2019): "Estimation with Aggregate Shocks," The Review of Economic Studies, forthcoming.

Hall, P., And C. C. Heyde (1980): Martingale limit theory and its application. Academic press.

HALl, R. E. (1978): "Stochastic Implications of the Life Cycle-Permanent Income Hypothesis: Theory and Evidence," Journal of Political Economy, 86(6), 971-87.

Hansen, L. P., and T. J. Sargent (1980): "Formulating and estimating dynamic linear rational expectations models," Journal of Economic Dynamics and Control, 2, 7-46.

(1982): "Instrumental variables procedures for estimating linear rational expectations models," Journal of Monetary Economics, 9(3), 263-296.

Hansen, L. P., and K. J. Singleton (1982): "Generalized instrumental variables estimation of nonlinear rational expectations models," Econometrica: Journal of the Econometric Society, pp. 1269-1286.

Hausman, J. A. (1996): Valuation of new goods under perfect and imperfect competitionchap. 5, pp. 209-248. University of Chicago Press.

Hendel, I., And A. Nevo (2006): "Measuring the Implications of Sales and Consumer Inventory Behavior," Econometrica, 74(6), 1637-1673.

Hotz, V. J., And R. A. Miller (1993): "Conditional Choice Probabilities and the Estimation of Dynamic Models," Review of Economic Studies, 60(3), 497-529.

Hotz, V. J., R. A. Miller, S. Sanders, And J. Smith (1994): "A Simulation Estimator for Dynamic Models of Discrete Choice," Review of Economic Studies, 61(2), 265-89.

Hu, Y., And M. Shum (2012): "Nonparametric identification of dynamic models with unobserved state variables," Journal of Econometrics, 171(1), 32-44.

Humphries, J. E. (2018): "The Causes and Consequences of Self-Employment over the Life Cycle," Discussion paper, Yale University.

Kalouptsidi, M. (2018): "Detection and Impact of Industrial Subsidies: The Case of Chinese Shipbuilding," Review of Economic Studies, 85(2), 1111-1158. 
Kalouptsidi, M., P. T. Scott, And E. A. SouzA-Rodrigues (2019): "Identification of Counterfactuals in Dynamic Discrete Choice Models," Quantitative Economics, forthcoming.

Kasahara, H., And K. Shimotsu (2009): "Nonparametric Identification of Finite Mixture Models of Dynamic Discrete Choices," Econometrica, 77(1), pp. 135-175.

Khorunzhina, N. (2013): "Structural estimation of stock market participation costs," Journal of Economic Dynamics and Control, 37(12), 2928-2942.

Khorunzhina, N., and R. A. Miller (2019): "American dream delayed: shifting determinants of home ownership," Discussion paper, Copenhagen Business School.

Kuersteiner, G. M., And I. R. Prucha (2013): "Limit theory for panel data models with cross sectional dependence and sequential exogeneity," Journal of Econometrics, 174(2), 107-126.

MA, L. (2019): "Learning in a hedonic framework: Valuing Brownfield remediation," International Economic Review, 60(3), 1355-1387.

Magnac, T., And D. Thesmar (2002): "Identifying Dynamic Discrete Decision Processes," Econometrica, 70(2), 801-816.

Matsumoto, B. (2016): "Lighting the fires: Explaining youth smoking initiation and experimentation in the context of a rational addiction model with learning," Discussion paper, U.S. Bureau of Labor Statistics.

Matzkin, R. L. (2003): "Nonparametric Estimation of Nonadditive Random Functions," Econometrica, 71(5), 1339-1375.

Melnikov, O. (2013): "Demand for differentiated durable products: The case of the us computer printer market," Economic Inquiry, 51(2), 1277-1298.

Montiel Olea, J., And C. Pflueger (2013): "A robust test for weak instruments," Journal of Business and Economic Statistics, 31, 358-369.

Morales, E., G. Sheu, And A. Zahler (2019): "Extended gravity," The Review of Economic Studies, 86(6), 2668-2712.

Nevo, A. (2000): "Mergers with differentiated products: The case of the ready-to-eat cereal industry," RAND Journal of Economics, 31, 395-421.

NeWey, W. K., AND J. L. POWELL (2003): "Instrumental variable estimation of nonparametric models," Econometrica, 71(5), 1565-1578.

Norets, A., And X. TAng (2014): "Semiparametric Inference in dynamic binary choice models," The Review of Economic Studies, 81(3), 1229-1262. 
PAKES, A. (2010): "Alternative models for moment inequalities," Econometrica, 78(6), 1783-1822.

Pesendorfer, M., And P. Schmidt-Dengler (2008): "Asymptotic Least Squares Estimators for Dynamic Games," The Review of Economic Studies, 75(3), 901-928.

Ransom, T. (2019): "Labor Market Frictions and Moving Costs of the Employed and Unemployed," Discussion Paper 12139, IZA Institute of Labor Economics.

Rust, J. (1987): "Optimal Replacement of GMC Bus Engines: an Empirical Model of Harold Zurcher," Econometrica, 55(5), 999-1033.

- (1994): "Structural estimation of Markov decision processes," Handbook of Econometrics, $4(4), 3081-3143$.

Rust, J., And C. Phelan (1997): "How social security and medicare affect retirement behavior in a world of incomplete markets," Econometrica, 65(4), 781-831.

RyAn, S. P. (2012): "The Costs of Environmental Regulation in a Concentrated Industry," Econometrica, 80(3), 1019-1061.

Schmidt, M.-A. (2019): "Valuing Flexibility: A Model of Discretionary Rest Breaks," Discussion paper, University of Toronto.

Scotт, P. T. (2013): "Dynamic Discrete Choice Estimation of Agricultural Land Use," Discussion paper, New York University.

Traiberman, S. (2019): "Occupations and Import Competition: Evidence from Denmark," American Economic Review, (109), 4260-4301.

Wright, B. (2014): "Global biofuels: key to the puzzle of grain market behavior," Journal of Economic Perspectives, 28(1), 73-98. 
Table 1: Parameters of the Monte Carlo Data Generating Process

\begin{tabular}{lccccc}
\hline \hline Payoff Parameters: & $\theta_{0}$ & 1 & $\xi \sim$ Normal AR $(1)$ & $\rho_{1}$ & 0 \\
& $\theta_{1}$ & -.1 & & $\rho_{2}$ & .2 \\
& & & & $\sigma_{\xi}^{2}$ & 0 or 16 \\
Prob. of Product Failure: & $\phi$ & .1 & & & \\
Discount Factor: & $\beta$ & .95 & & $\rho_{3}$ & 0 \\
Process for price $w_{m t}:$ & $\gamma_{0}$ & 40 & & $\rho_{4}$ & .7 \\
& $\gamma_{1}$ & 1 & Aggregate Shocks & $\lambda_{z}$ & 0 or .7 \\
& $\gamma_{2}$ & 1 & & & \\
& $\sigma_{w}^{2}$ & 4 & & & \\
\hline
\end{tabular}


Table 2: Monte Carlo Experiments without Unobserved Demand Shock $\sigma_{\xi}^{2}=0 \quad$ True Parameters: $\theta_{0}=1, \theta_{1}=-.1$

\begin{tabular}{|c|c|c|c|c|c|c|c|c|c|}
\hline \multirow{3}{*}{ Estimator } & & \multirow{3}{*}{$\begin{array}{l}\mathrm{T} \\
\mathrm{M}\end{array}$} & \multicolumn{3}{|c|}{$\lambda_{z}=0$} & \multicolumn{4}{|c|}{$\lambda_{z}=.7$} \\
\hline & & & 40 & 160 & 10 & 40 & 160 & 10 & 160 \\
\hline & & & 40 & 10 & 160 & 40 & 10 & 160 & 160 \\
\hline \multirow{8}{*}{$\begin{array}{l}\text { ECCP: } \\
\text { OLS }\end{array}$} & \multirow[t]{4}{*}{$\theta_{0}$} & Mean Est. & 1.01 & 1.01 & 1.01 & 0.92 & 0.99 & 0.75 & 0.99 \\
\hline & & Rel. Bias & $0.64 \%$ & $0.74 \%$ & $0.81 \%$ & $-8.05 \%$ & $-1.49 \%$ & $-24.6 \%$ & $-1.38 \%$ \\
\hline & & $\mathrm{SD}$ & 0.04 & 0.04 & 0.04 & 0.17 & 0.09 & 0.29 & 0.09 \\
\hline & & RMSE & 0.04 & 0.04 & 0.04 & 0.19 & 0.10 & 0.38 & 0.09 \\
\hline & \multirow[t]{4}{*}{$\theta_{1}$} & Mean Est. & -0.10 & -0.10 & -0.10 & -0.10 & -0.10 & -0.09 & -0.10 \\
\hline & & Rel. Bias & $0.16 \%$ & $0.18 \%$ & $0.20 \%$ & $-1.98 \%$ & $-0.38 \%$ & $-6.20 \%$ & $-0.33 \%$ \\
\hline & & $\mathrm{SD}$ & $1.01 \mathrm{e}-3$ & $1.04 \mathrm{e}-3$ & $9.97 \mathrm{e}-4$ & $4.05 \mathrm{e}-3$ & $2.22 \mathrm{e}-3$ & $6.78 \mathrm{e}-3$ & $2.05 \mathrm{e}-3$ \\
\hline & & RMSE & $1.02 \mathrm{e}-3$ & $1.06 \mathrm{e}-3$ & $1.02 \mathrm{e}-3$ & $4.50 \mathrm{e}-3$ & $2.26 \mathrm{e}-3$ & $9.19 \mathrm{e}-3$ & $2.07 \mathrm{e}-3$ \\
\hline \multirow{8}{*}{$\begin{array}{l}\text { ECCP: } \\
\text { IV (2SLS) }\end{array}$} & \multirow[t]{4}{*}{$\theta_{0}$} & Mean Est. & 1.01 & 1.01 & 1.01 & 0.91 & 0.98 & 0.72 & 0.98 \\
\hline & & Rel. Bias & $0.70 \%$ & $0.79 \%$ & $0.87 \%$ & $-9.0 \%$ & $-1.66 \%$ & $-28.2 \%$ & $-1.57 \%$ \\
\hline & & $\mathrm{SD}$ & 0.04 & 0.04 & 0.04 & 0.19 & 0.10 & 0.33 & 0.09 \\
\hline & & RMSE & 0.04 & 0.05 & 0.04 & 0.21 & 0.10 & 0.43 & 0.10 \\
\hline & \multirow[t]{4}{*}{$\theta_{1}$} & Mean Est. & -0.10 & -0.10 & -0.10 & -0.10 & -0.10 & -0.09 & -0.10 \\
\hline & & Rel. Bias & $0.18 \%$ & $0.19 \%$ & $0.22 \%$ & $-2.22 \%$ & $-0.42 \%$ & $-7.08 \%$ & $-0.38 \%$ \\
\hline & & $\mathrm{SD}$ & $1.06 \mathrm{e}-3$ & $1.08 \mathrm{e}-3$ & $1.03 \mathrm{e}-3$ & $4.46 \mathrm{e}-3$ & $2.40 \mathrm{e}-3$ & $7.67 \mathrm{e}-3$ & $2.23 \mathrm{e}-3$ \\
\hline & & RMSE & $1.07 \mathrm{e}-3$ & $1.10 \mathrm{e}-3$ & $1.06 \mathrm{e}-3$ & $4.98 \mathrm{e}-3$ & $2.44 \mathrm{e}-3$ & 0.01 & $2.26 \mathrm{e}-3$ \\
\hline \multirow{8}{*}{$\begin{array}{l}\text { Standard } \\
\text { CCP }\end{array}$} & \multirow[t]{4}{*}{$\theta_{0}$} & Mean Est. & 1.00 & 1.00 & 1.00 & 1.00 & 1.00 & 1.00 & 1.00 \\
\hline & & Rel. Bias & $-0.29 \%$ & $-0.27 \%$ & $-0.11 \%$ & $-0.49 \%$ & $-0.31 \%$ & $-0.02 \%$ & $-0.19 \%$ \\
\hline & & $\mathrm{SD}$ & $9.22 \mathrm{e}-3$ & $8.45 \mathrm{e}-3$ & 0.01 & 0.04 & 0.02 & 0.07 & 0.02 \\
\hline & & RMSE & $9.66 \mathrm{e}-3$ & 8.87e-3 & 0.01 & 0.04 & 0.02 & 0.07 & 0.02 \\
\hline & \multirow[t]{4}{*}{$\theta_{1}$} & Mean Est. & -0.10 & -0.10 & -0.10 & -0.10 & -0.10 & -0.10 & -0.10 \\
\hline & & Rel. Bias & $-0.48 \%$ & $-0.51 \%$ & $-0.30 \%$ & $-0.70 \%$ & $-0.60 \%$ & $-0.64 \%$ & $-0.25 \%$ \\
\hline & & $\mathrm{SD}$ & $4.40 \mathrm{e}-4$ & $3.88 \mathrm{e}-4$ & $6.41 \mathrm{e}-4$ & $7.96 \mathrm{e}-4$ & $5.27 \mathrm{e}-4$ & $1.33 \mathrm{e}-3$ & $5.18 \mathrm{e}-4$ \\
\hline & & RMSE & $6.48 \mathrm{e}-4$ & $6.42 \mathrm{e}-4$ & $7.08 \mathrm{e}-4$ & $1.06 \mathrm{e}-3$ & $7.95 \mathrm{e}-4$ & $1.48 \mathrm{e}-3$ & $5.73 \mathrm{e}-4$ \\
\hline
\end{tabular}

Notes: 5000 Monte Carlo replications for each sample structure. SD is the standard deviation of the estimators across replications. RMSE is root-mean squared error. Relative Bias is bias as percentage of the true parameter. 
Table 3: Monte Carlo Experiments with Unobserved Demand Shock $\sigma_{\xi}^{2}=16 \quad$ True Parameters: $\theta_{0}=1, \theta_{1}=-.1$

\begin{tabular}{|c|c|c|c|c|c|c|c|c|c|}
\hline \multirow[b]{2}{*}{ Estimator } & \multirow{2}{*}{\multicolumn{2}{|c|}{$\begin{array}{l}\mathrm{T} \\
\mathrm{M}\end{array}$}} & \multicolumn{3}{|c|}{$\lambda_{z}=0$} & \multicolumn{4}{|c|}{$\lambda_{z}=.7$} \\
\hline & & & $\begin{array}{l}40 \\
40\end{array}$ & $\begin{array}{r}160 \\
10\end{array}$ & $\begin{array}{r}10 \\
160\end{array}$ & $\begin{array}{l}40 \\
40\end{array}$ & $\begin{array}{r}160 \\
10\end{array}$ & $\begin{array}{r}10 \\
160\end{array}$ & $\begin{array}{l}160 \\
160\end{array}$ \\
\hline $\begin{array}{l}\text { ECCP: } \\
\text { OLS }\end{array}$ & $\theta_{0}$ & $\begin{array}{l}\text { Mean Est. } \\
\text { Rel. Bias } \\
\text { SD } \\
\text { RMSE }\end{array}$ & $\begin{array}{r}-8.62 \\
-962 \% \\
0.59 \\
9.64\end{array}$ & $\begin{array}{r}-8.63 \\
-963 \% \\
0.58 \\
9.64\end{array}$ & $\begin{array}{r}-8.61 \\
-961 \% \\
0.57 \\
9.62\end{array}$ & $\begin{array}{r}-9.54 \\
-1050 \% \\
1.79 \\
10.70\end{array}$ & $\begin{array}{r}-8.81 \\
-981 \% \\
1.03 \\
9.86\end{array}$ & $\begin{array}{r}-11.60 \\
-1260 \% \\
2.77 \\
12.90\end{array}$ & $\begin{array}{r}-8.85 \\
-985 \% \\
0.89 \\
9.89\end{array}$ \\
\hline & $\theta_{1}$ & $\begin{array}{l}\text { Mean Est. } \\
\text { Rel. Bias } \\
\text { SD } \\
\text { RMSE }\end{array}$ & $\begin{array}{r}0.14 \\
-240 \% \\
0.01 \\
0.24\end{array}$ & $\begin{array}{r}0.14 \\
-241 \% \\
0.01 \\
0.24\end{array}$ & $\begin{array}{r}0.14 \\
-240 \% \\
0.01 \\
0.24\end{array}$ & $\begin{array}{r}0.16 \\
-264 \% \\
0.04 \\
0.27\end{array}$ & $\begin{array}{r}0.15 \\
-245 \% \\
0.02 \\
0.25\end{array}$ & $\begin{array}{r}0.22 \\
-315 \% \\
0.06 \\
0.32\end{array}$ & $\begin{array}{r}0.15 \\
-246 \% \\
0.02 \\
0.25\end{array}$ \\
\hline $\begin{array}{l}\text { ECCP: } \\
\text { IV (2SLS) }\end{array}$ & $\theta_{0}$ & $\begin{array}{l}\text { Mean Est. } \\
\text { Rel. Bias } \\
\text { SD } \\
\text { RMSE }\end{array}$ & $\begin{array}{r}1.02 \\
1.62 \% \\
0.77 \\
0.77\end{array}$ & $\begin{array}{r}1.00 \\
-0.20 \% \\
0.77 \\
0.77\end{array}$ & $\begin{array}{r}1.02 \\
1.82 \% \\
0.75 \\
0.75\end{array}$ & $\begin{array}{r}0.97 \\
-3.49 \% \\
0.83 \\
0.83\end{array}$ & $\begin{array}{r}1.02 \\
1.97 \% \\
0.78 \\
0.78\end{array}$ & $\begin{array}{r}0.87 \\
-12.8 \% \\
0.90 \\
0.91\end{array}$ & $\begin{array}{r}0.99 \\
-0.76 \% \\
0.20 \\
0.20\end{array}$ \\
\hline & $\theta_{1}$ & $\begin{array}{l}\text { Mean Est. } \\
\text { Rel. Bias } \\
\text { SD } \\
\text { RMSE }\end{array}$ & $\begin{array}{r}-0.10 \\
0.50 \% \\
0.02 \\
0.02\end{array}$ & $\begin{array}{r}-0.10 \\
-0.05 \% \\
0.02 \\
0.02\end{array}$ & $\begin{array}{r}-0.10 \\
0.42 \% \\
0.02 \\
0.02\end{array}$ & $\begin{array}{r}-0.10 \\
-1.00 \% \\
0.02 \\
0.02\end{array}$ & $\begin{array}{r}-0.10 \\
0.44 \% \\
0.02 \\
0.02\end{array}$ & $\begin{array}{r}-0.10 \\
-3.25 \% \\
0.02 \\
0.02\end{array}$ & $\begin{array}{r}-0.10 \\
-0.19 \% \\
4.96 \mathrm{e}-3 \\
4.96 \mathrm{e}-3\end{array}$ \\
\hline $\begin{array}{l}\text { Standard } \\
\mathrm{CCP}\end{array}$ & $\theta_{0}$ & $\begin{array}{l}\text { Mean Est. } \\
\text { Rel. Bias } \\
\text { SD } \\
\text { RMSE }\end{array}$ & $\begin{array}{r}0.26 \\
-74.4 \% \\
0.03 \\
0.75\end{array}$ & $\begin{array}{r}0.26 \\
-74.4 \% \\
0.03 \\
0.75\end{array}$ & $\begin{array}{r}0.25 \\
-74.7 \% \\
0.03 \\
0.75\end{array}$ & $\begin{array}{r}0.26 \\
-74.3 \% \\
0.04 \\
0.74\end{array}$ & $\begin{array}{r}0.26 \\
-74.3 \% \\
0.03 \\
0.74\end{array}$ & $\begin{array}{r}0.26 \\
-73.5 \% \\
0.05 \\
0.74\end{array}$ & $\begin{array}{r}0.17 \\
-83.3 \% \\
0.02 \\
0.83\end{array}$ \\
\hline & $\theta_{1}$ & $\begin{array}{l}\text { Mean Est. } \\
\text { Rel. Bias } \\
\text { SD } \\
\text { RMSE }\end{array}$ & $\begin{array}{r}-0.01 \\
-88.1 \% \\
5.38 \mathrm{e}-3 \\
0.09\end{array}$ & $\begin{array}{r}-0.01 \\
-87.8 \% \\
5.12 \mathrm{e}-3 \\
0.09\end{array}$ & $\begin{array}{r}-0.01 \\
-89.2 \% \\
5.74 \mathrm{e}-3 \\
0.09\end{array}$ & $\begin{array}{r}-0.01 \\
-88.4 \% \\
6.33 \mathrm{e}-3 \\
0.09\end{array}$ & $\begin{array}{r}-0.01 \\
-87.8 \% \\
5.41 \mathrm{e}-3 \\
0.09\end{array}$ & $\begin{array}{r}-0.01 \\
-89.9 \% \\
9.24 \mathrm{e}-3 \\
0.09\end{array}$ & $\begin{array}{r}2.12 \mathrm{e}-3 \\
-102 \% \\
3.66 \mathrm{e}-3 \\
0.10\end{array}$ \\
\hline
\end{tabular}

Notes: 5000 Monte Carlo replications for each sample structure. SD is the standard deviation of the estimators across replications. RMSE is root-mean squared error. Relative Bias is bias as percentage of the true parameter. 
Table 4: Summary Statistics

\begin{tabular}{lccc}
\hline Hour of the Day & $\begin{array}{c}\text { Share of Drivers that } \\
\text { Continue Working (\%) }\end{array}$ & \multicolumn{2}{c}{ Hourly Earnings $(\$)$} \\
Mean & Std Dev \\
\hline & 100 & 32.68 & 3.14 \\
6:00 AM & 100 & 35.14 & 3.01 \\
7:00 AM & 98.12 & 37.20 & 3.10 \\
8:00 AM & 97.60 & 34.70 & 2.44 \\
9:00 AM & 96.45 & 29.97 & 2.19 \\
10:00 AM & 95.04 & 29.21 & 2.27 \\
11:00 AM & 94.88 & 30.96 & 2.23 \\
12:00 PM & 94.28 & 30.80 & 2.24 \\
1:00 PM & 91.49 & 33.83 & 2.21 \\
2:00 PM & 79.39 & 34.98 & 1.98 \\
3:00 PM & 51.37 & 24.84 & 2.09 \\
4:00 PM & 0 & - \\
5:00 PM & \multicolumn{4}{c}{3937} \\
\hline Number of Drivers & \multicolumn{3}{c}{209} \\
Number of Days &
\end{tabular}

Notes: The unit of observation is a taxi driver working in a given hour of a week day (Monday-Thursday) in 2013. The sample is restricted to day shifts and drivers working for at least one hour and at most twelve hours. The numbers in the cells are sample averages (and standard deviations) across drivers and days for each hour of the day.

Source: authors' calculations. 
Table 5: Parameter Estimates

\begin{tabular}{lccc}
\hline & $(1)$ & $(2)$ & $(3)$ \\
& OLS & IV - Within Day Lagged & IV - Previous Day \\
\hline Hourly Earnings $\left(\theta_{1}\right)$ & 0.00358 & 0.00672 & \\
& $(0.00201)$ & $(0.00346)$ & 0.0223 \\
& & & $(0.00621)$ \\
Hours Driving $\left(\theta_{2}\right)$ & -0.291 & -0.291 & -0.291 \\
& $(0.00462)$ & $(0.00461)$ & $(0.00462)$ \\
\hline F-statistic & - & 3066 & 105.4 \\
Number of Days & 209 & 209 & 208 \\
Number of Hours & 12 & 11 & 12 \\
Observations & 2508 & 2299 & 2496 \\
\hline
\end{tabular}

Notes: The unit of observation is an hour $(t)$ of a day $(m)$ in 2013. The dependent variable is the $\log$ odds ratio of the conditional choice probabilities of continue working versus stopping at $t$ plus the discounted choice probability of stop working at $t+1$. The first column presents the OLS estimates. The second column, the 2SLS estimates using the within-day lagged earnings $\left(w_{m t-1}\right)$ as an instrument for hourly earnings $\left(w_{m t}\right)$. And the third column, the 2SLS estimates using the earnings in the same hour of the day but in the previous day $\left(w_{m-1, t}\right)$ as the instrument. All specifications include dummy variables for (i) important holidays, (ii) hourly weather conditions, and (iii) the hour of the day, as explained in the main text (omitted from the table). Robust Standard errors in parentheses are clustered at the 'market' level $m$ (correponding to a day in 2013). 


\title{
Supplement to "Linear IV Regression Estimators for Structural Dynamic Discrete Choice Models
}

\author{
Myrto Kalouptsidi, Paul T. Scott $\dagger$ Eduardo Souza-Rodrigues ${ }^{\ddagger}$ \\ Harvard University, CEPR and NBER; NYU Stern; University of Toronto
}

February 2020

This supplemental material consists of the following sections: Section A presents general derivations of Euler equations for both deterministic and stochastic finite dependence. It also extends Proposition 2 to allow for stochastic sequences of choices (see Proposition A1). Sections B and C present all proofs of the lemmas and propositions presented in the main paper; Section B focuses on the identification results, while Section $\mathrm{C}$ shows the proofs of the asymptotic properties of the ECCP estimator. Section D explains the standard CCP approach implemented in the Monte Carlo experiment to estimate the model parameters. Finally, Section E extends the Monte Carlo study presented in Section 6 of the main paper by investigating how the biases in the parameter estimates pass through to biases in the countefactuals calculations.

\section{A General ECCP Equation Derivation}

In this section, we offer a general derivation of Euler equations in conditional choice probabilities relying first on deterministic finite dependence as defined in Section 3, and then exploring stochastic sequences of choices. We also present Proposition A1, which extends the identification result in Proposition 2 (presented in the main text) to allow for stochastic finite dependence. Recall that finite dependence is not a behavioral assumption (whether based on deterministic or stochastic sequences); it is rather a property that the state transition process may or may not satisfy in the data.

*Harvard University, Department of Economics, Littauer Center 124, Cambridge, MA 02138. email: myrto@fas.harvard.edu

†Stern School of Business, New York University, Kaufman Management Center, 44 W. 4th St., New York, NY 10012. email: pscott@stern.nyu.edu.

${ }_{\ddagger}^{\ddagger}$ Department of Economics, University of Toronto, Max Gluskin House, 150 St. George St., Toronto, Ontario M5S 3G7, Canada. email: e.souzarodrigues@utoronto.ca 


\section{A.1 ECCP Equation Under Deterministic Finite Dependence}

Arcidiacono and Miller (2011) show that the conditional value functions, $v_{a}$, can be represented by functions of flow payoffs and conditional choice probabilities for any sequence of future choices, optimal or not. To derive such a representation, begin with an arbitrary initial state $\omega_{m t}$. Consider a sequence of actions from $t$ to $t+\tau$ (where $\tau \geq 1$ ). Suppose the initial choice at time period $t$ is $a$, and let $j$ denote another element of the choice set $\mathcal{A}$. Let $a_{d} \in \mathcal{A}$ denote the $d$-th choice in the sequence following $a$, and define $j_{d} \in \mathcal{A}$ similarly.

Recall equation (11), which is a vector across rows of the individual state $k$ and absorbs the aggregate state $\omega_{m t}$ into $m t$ subscripts:

$$
\pi_{a m t}+\beta e_{a m, t, t+1}^{V}=V_{m t}-\beta F_{a m t}^{k} V_{m t+1}-\psi_{a m t}
$$

We then substitute for $V_{m t+1}$ using equation (11) again, using $a_{1}$ as the action instead of $a$ :

$$
\begin{aligned}
\pi_{a m t}+\beta e_{a m, t, t+1}^{V}= & V_{m t}-\psi_{a m t}-\beta F_{a m t}^{k}\left(\pi_{a_{1} m t+1}+\beta e_{a_{1}, m, t+1, t+2}^{V}+\psi_{a_{1} m t+1}\right) \\
& -\beta^{2} F_{a m t}^{k} F_{a_{1} m t+1}^{k} V_{m t+2} .
\end{aligned}
$$

Repeated substitution of $V_{m t+d}$ above leads to:

$$
\begin{aligned}
\pi_{a m t}+\beta e_{a m, t, t+1}^{V}= & V_{m t}-\psi_{a m t} \\
& -F_{a m t}^{k}\left[\sum_{d=1}^{\tau} \beta^{d} \Lambda_{a m t d}\left(\pi_{a_{d} m t+d}+\beta e_{a_{d}, m, t+d, t+d+1}^{V}+\psi_{a_{d} m t+d}\right)\right] \\
& -\beta^{\tau+1} F_{a m t}^{k} \Lambda_{a m t, \tau+1} V_{m t+\tau+1}
\end{aligned}
$$

where the matrices $\Lambda_{a m t d}$ are defined recursively:

$$
\begin{array}{llcl}
\Lambda_{a m t d}= & I, & \text { for } d=1, \\
\Lambda_{a m t d}= & \Lambda_{a m t, d-1} F_{a_{d-1} m t+d-1}^{k}, & \text { for } d \geq 2,
\end{array}
$$

Next, finite dependence allows us to eliminate the $V_{m t+\tau+1}$, resulting in an ECCP equation that forms the basis of our identification arguments. Recall Definition 2 in Section 3: Given $\tau$ period finite dependence, for a pair of actions $(a, j)$, we can construct sequences $\left(a, a_{1}, \ldots, a_{\tau}\right)$ and $\left(j, j_{1}, \ldots, j_{\tau}\right)$ such that ${ }^{1}$

$$
F_{a m t}^{k} F_{a_{1} m t+1}^{k} \ldots F_{a_{\tau} m t+\tau}^{k}=F_{j m t}^{k} F_{j_{1} m t+1}^{k} \ldots F_{j_{\tau} m t+\tau}^{k}
$$

i.e.,

$$
F_{a m t}^{k} \Lambda_{a m t, \tau+1}=F_{j m t}^{k} \Lambda_{j m t, \tau+1} .
$$

\footnotetext{
${ }^{1}$ Recall that the terms in the sequences depend on the particular initial pair of actions $(a, j)$ chosen.
} 
We then difference equation (A1) across the two sequences of actions. Because of (A2), the last term cancels, and the result is equation (27).

\section{A.2 ECCP Equation Under Stochastic Finite Dependence}

Consider now a known mixing sequence of actions from $t$ to $t+\tau$ (where $\tau \geq 1$ ). Suppose the initial choice at $t$ is action $a$. For the next period, $t+1$, let $\alpha_{t+1}^{a}\left(k_{i m t+1}, \omega_{m t+1}\right)$ be a vector on $\mathbb{R}^{A+1}$ with elements $\alpha_{l t+1}^{a}\left(k_{i m t+1}, \omega_{m t+1}\right), l \in \mathcal{A}$, such that $\sum_{l=0}^{A} \alpha_{l t+1}^{a}\left(k_{i m t+1}, \omega_{m t+1}\right)=1$. Each element $\alpha_{l t+1}^{a}\left(k_{i m t+1}, \omega_{m t+1}\right)$ of the vector $\alpha_{t+1}^{a}$ can be interpreted as the weight given to action $l \in \mathcal{A}$ in period $t+1$ at state $\left(k_{i m t}, \omega_{m t}\right)$ after the initial choice $a$ at $t$. For deterministic sequences, one element of the vector $\alpha_{t+1}^{a}$ equals one and the others equal zero. For probabilistic sequences, all elements of $\alpha_{t+1}^{a}$ are positive an add up to one. More generally, the mixing may involve negative weights, provided that they sum up to one (Arcidiacono and Miller, 2019). For the other time periods, $d=2, \ldots, \tau$, take the sequence of weight choices in a similar way, $\alpha_{t+d}^{a}\left(k_{i m t+d}, \omega_{m t+d}\right)$. In this section, we abuse terminology and use the terms "mixing" and "stochastic" interchangeably.

It is useful to represent the weight choices in matrix notation. Define the diagonal matrix $\alpha_{l m t}^{a}=\operatorname{diag}\left\{\alpha_{l t}^{a}\left(k, \omega_{m t}\right) ; k \in \mathcal{K}\right\}$, for $l \in \mathcal{A}$ and any time period $t$. In words, $\alpha_{l m t}^{a}$ is a $K \times K$ matrix that collects the individual terms $\alpha_{l t}^{a}\left(k_{i m t}, \omega_{m t}\right)$ for all possible values of $k$. Note that $\alpha_{l t}^{a}\left(k_{i m t}, \omega_{m t}\right)$ is one element of the vector $\alpha_{t}^{a}\left(k_{i m t}, \omega_{m t}\right)$, as defined in the previous paragraph. Clearly, because the mixing requires $\sum_{l=0}^{A} \alpha_{l t}^{a}\left(k_{i m t}, \omega_{m t}\right)=1$, we have that $\sum_{l=0}^{A} \alpha_{l m t}^{a}=I_{K}$, where $I_{K}$ is the $K \times K$ identity matrix.

Now, recall equation (11). For any initial choice $a$, take a mixing $\alpha_{t+1}^{a}\left(k_{i m t+1}, \omega_{m t+1}\right)$ over choices in $\mathcal{A}$ at $t+1$, and replace $V_{m t+1}$ in (11) to get

$$
\begin{aligned}
\pi_{a m t}+\beta e_{a m, t, t+1}^{V}= & V_{m t}-\psi_{a m t}-\beta F_{a m t}^{k}\left[\sum_{l=0}^{A} \alpha_{l m t+1}^{a}\left(\pi_{l m t+1}+\beta e_{l m, t+1, t+2}^{V}+\psi_{l m t+1}\right)\right] \\
& -\beta^{2} F_{a m t}^{k}\left[\sum_{l=0}^{A} \alpha_{l m t+1}^{a} F_{l m t+1}^{k}\right] V_{m t+2} .
\end{aligned}
$$

Next, we follow the steps outlined in Section A.1 of this Appendix. Repeated substitution of $V_{m t+d}$ above leads to:

$$
\begin{aligned}
\pi_{a m t}+\beta e_{a m, t, t+1}^{V}= & V_{m t}-\psi_{a m t} \\
& -\beta F_{a m t}^{k}\left[\sum_{d=1}^{\tau} \beta^{d-1} \Lambda_{a m, t, d} \sum_{l=0}^{A} \alpha_{l m t+d}^{a}\left(\pi_{l m t+d}+\beta e_{l m, t+d, t+d+1}^{V}+\psi_{l m t+d}\right)\right] \\
& -\beta^{\tau+1} F_{a m t}^{k} \Lambda_{a m, t, \tau+1} V_{m t+\tau+1},
\end{aligned}
$$


where the observed (estimable) matrices $\Lambda_{a m, t, d}$ are defined recursively:

$$
\begin{aligned}
& \Lambda_{a m, t, d}=\quad I, \quad \text { for } d=1 \\
& \Lambda_{a m, t, d}=\Lambda_{a m, t, d-1}\left[\sum_{l=0}^{A} \alpha_{l m t+d-1}^{a} F_{l m t+d-1}^{k}\right], \text { for } d \geq 2 \text {. }
\end{aligned}
$$

As before, we make use of finite dependence to eliminate the $V_{m t+\tau+1}$. First, we extend Definition 2 in Section 3 to stochastic sequences of choices:

Definition 1. (Stochastic Finite Dependence) A pair of choices $a$ and $j$ satisfies stochastic $\tau$-period finite dependence if there exists two sequences of mixings starting at $a$ and $j$ such that, for all $t$,

$$
F_{a m t}^{k} \Lambda_{a m, t, \tau+1}=F_{j m t}^{k} \Lambda_{j m, t, \tau+1}
$$

where $\Lambda_{a m, t, \tau+1}$ is defined in (A4).

Under this condition, $V_{m t+\tau+1}$ is eliminated when we difference the equation above across two mixing sequences of actions, starting respectively at $a$ and $j$. Recalling that $\pi=\bar{\pi}+\xi$, we then obtain the ECCP regression equation:

$$
\begin{aligned}
& \psi_{j m t}-\psi_{a m t}+\beta \sum_{d=1}^{\tau} \sum_{l=0}^{A} \beta^{d-1}\left[F_{j m t}^{k} \Lambda_{j m, t, d} \alpha_{l m t+d}^{j}-F_{a m t}^{k} \Lambda_{a m, t, d} \alpha_{l m t+d}^{a}\right] \psi_{l m t+d} \\
& =\bar{\pi}_{a m t}-\bar{\pi}_{j m t}+\beta \sum_{d=1}^{\tau} \sum_{l=0}^{A} \beta^{d-1}\left[F_{a m t}^{k} \Lambda_{a m, t, d} \alpha_{l m t+d}^{a}-F_{j m t}^{k} \Lambda_{j m, t, d} \alpha_{l m t+d}^{j}\right] \bar{\pi}_{l m t+d} \\
& +u_{a j m t},
\end{aligned}
$$

where the econometric error term is $u_{\text {ajmt }}=\widetilde{\xi}_{\text {ajmt }}+\widetilde{e}_{\text {ajmt }}^{V}$, with

$$
\widetilde{\xi}_{a j m t}=\xi_{a m t}-\xi_{j m t}+\beta \sum_{d=1}^{\tau} \sum_{l=0}^{A} \beta^{d-1}\left[F_{a m t}^{k} \Lambda_{a m, t, d} \alpha_{l m t+d}^{a}-F_{j m t}^{k} \Lambda_{j m, t, d} \alpha_{l m t+d}^{j}\right] \xi_{l m t+d}
$$

and

$\widetilde{e}_{a j m t}^{V}=\beta\left(e_{a m, t, t+1}^{V}-e_{j m, t, t+1}^{V}\right)+\beta \sum_{d=1}^{\tau} \sum_{l=0}^{A} \beta^{d}\left[F_{a m t}^{k} \Lambda_{a m, t, d} \alpha_{l m t+d}^{a}-F_{j m t}^{k} \Lambda_{j m, t, d} \alpha_{l m t+d}^{j}\right] e_{l m, t+d, t+d+1}^{V}$.

We can now extend Proposition 2 to identify payoff parameters under the assumption of general stochastic finite dependence.

Proposition A1. Suppose Assumptions 1 and 2 hold. Assume $\tau$-period stochastic finite dependence holds for the agent-level transition process $F^{k}$, with $\tau<T$. Assume also a linear-inparameters flow payoff: $\bar{\pi}(a, k, w)=x(a, k, w) \theta$, where $\theta \in \mathbb{R}^{P}$, and $x(a, k, w)$ is a known (vector) 
function. Let $X_{a m t}$ be a $K \times P$ matrix with elements given by $x\left(a, k, w_{m t}\right)$, so that $\bar{\pi}_{a m t}=X_{a m t} \theta$, and define

$$
\widetilde{X}_{a j m t} \equiv X_{a m t}-X_{j m t}+\beta \sum_{d=1}^{\tau} \sum_{l=0}^{A} \beta^{d-1}\left[F_{a m t}^{k} \Lambda_{a m, t, d} \alpha_{l m t+d}^{a}-F_{j m t}^{k} \Lambda_{j m, t, d} \alpha_{l m t+d}^{j}\right] X_{l m t+d}
$$

Denote the $K \times 1$ vector on the left hand side of (A6) by $Y_{\text {ajmt }}$. Stack equation (A6) for all feasible combinations of actions $(a, j) \in \mathcal{A}$ to obtain the following equation

$$
Y_{m t}=\widetilde{X}_{m t} \theta+u_{m t}
$$

where $Y_{m t}, \widetilde{X}_{m t}$, and $u_{m t}$ stack the vectors $Y_{\text {ajmt }}, \widetilde{X}_{\text {ajmt }}$ and $u_{\text {ajmt }}$, respectively. Let $Z_{m t}$ be an $L \times K$ matrix of instrumental variables with $L \geq P$. The parameter $\theta$ is identified provided $E\left[Z_{m t} u_{m t}\right]=0$ and $\operatorname{rank}\left(E\left[Z_{m t} \tilde{X}_{m t}\right]\right)=P$.

The proof of Proposition A1 is identical to the proof of Proposition 2 and is therefore omitted. In fact, Proposition 2 is a special case of Proposition A1, when finite dependence is restricted to satisfy deterministic sequences of actions.

Proposition A1 can be used to extend previous empirical applications exploring stochastic finite dependence (e.g., Ransom, 2019) to incorporate serially correlated unobservable states, measurement error, and endogeneity problems. It can also serve as the basis for identification arguments in future applications featuring all these attributes. Evidently, the same set of issues discussed extensively in Section 4 involving deterministic finite dependence (regarding instrument validity, limitations of and extensions to the ECCP approach) applies here as well.

\section{B Proofs: Identification}

\section{B.1 Proof of Proposition 1}

Assume single-action $\tau$-period dependence holds for action $J$. Then, equation (27) simplifies to

$$
\begin{aligned}
& \left(\psi_{j m t}-\psi_{a m t}\right)+\left(F_{j m t}^{k}-F_{a m t}^{k}\right) \sum_{d=1}^{\tau} \beta^{d} \Lambda_{J m t d} \psi_{J m t+d} \\
& =\bar{\pi}_{a m t}-\bar{\pi}_{j m t}-\left(F_{j m t}^{k}-F_{a m t}^{k}\right) \sum_{d=1}^{\tau} \beta^{d} \Lambda_{J m t d} \bar{\pi}_{J m t+d}+u_{a j m t},
\end{aligned}
$$

where the matrix $\Lambda_{J m t d}$ is defined recursively

$$
\begin{aligned}
& \Lambda_{\text {Jmtd }}=\quad I, \quad \text { for } d=1 \\
& \Lambda_{J m t d}=\Lambda_{J m t, d-1} F_{J m t+d-1}^{k}, \text { for } d \geq 2 \text {, }
\end{aligned}
$$


and the unobservable term is $u_{\text {ajmt }}=\widetilde{\xi}_{\text {ajmt }}+\widetilde{e}_{\text {ajmt }}^{V}$, with

$$
\begin{aligned}
& \widetilde{\xi}_{a j m t}=\left(\xi_{a m t}-\xi_{j m t}\right)-\left(F_{j m t}^{k}-F_{a m t}^{k}\right) \sum_{d=1}^{\tau} \beta^{d} \Lambda_{J m t d} \xi_{J m t+d}, \\
& \widetilde{e}_{a j m t}^{V}=\beta\left(e_{a m, t, t+1}^{V}-e_{j m, t, t+1}^{V}\right)-\left(F_{j m t}^{k}-F_{a m t}^{k}\right) \sum_{d=1}^{\tau} \beta^{d} \Lambda_{J m t d} e_{J m, t+d, t+d+1}^{V} .
\end{aligned}
$$

For any known (and comformable) function $h\left(z_{m t}\right)$, multiply both sides of (B1) and take the expectation. We eliminate the error terms $\widetilde{\xi}_{a j m t}$ and $\widetilde{e}_{\text {ajmt }}^{V}$ by Assumption 3.(ii)-(iii). Then,

$$
\begin{aligned}
& E\left[h\left(z_{m t}\right)\left(\left(\psi_{j m t}-\psi_{a m t}\right)+\left(F_{j m t}^{k}-F_{a m t}^{k}\right) \sum_{d=1}^{\tau} \beta^{d} \Lambda_{J m t d} \psi_{J m t+d}\right)\right] \\
= & E\left[h\left(z_{m t}\right)\left(\bar{\pi}_{a m t}-\bar{\pi}_{j m t}-\left(F_{j m t}^{k}-F_{a m t}^{k}\right) \sum_{d=1}^{\tau} \beta^{d} \Lambda_{J m t d} \bar{\pi}_{J m t+d}\right)\right],
\end{aligned}
$$

where the expectations are taken over $\left(z_{m t}, w_{m t}, \ldots, w_{m t+\tau}\right)$.

The LHS of (B4) can be recovered from the data (using the results of Lemma C1, in Appendix C.4). Then, for any two primitives $\bar{\pi}$ and $\bar{\pi}^{\prime}$,

$$
\begin{aligned}
& E\left[h\left(z_{m t}\right)\left(\bar{\pi}_{a m t}-\bar{\pi}_{j m t}-\left(F_{j m t}^{k}-F_{a m t}^{k}\right) \sum_{d=1}^{\tau} \beta^{d} \Lambda_{J m t d} \bar{\pi}_{J m t+d}\right)\right] \\
= & E\left[h\left(z_{m t}\right)\left(\bar{\pi}_{a m t}^{\prime}-\bar{\pi}_{j m t}^{\prime}-\left(F_{j m t}^{k}-F_{a m t}^{k}\right) \sum_{d=1}^{\tau} \beta^{d} \Lambda_{J m t d} \bar{\pi}_{J m t+d}^{\prime}\right)\right] .
\end{aligned}
$$

By the completeness condition (Assumption 3.(i)),

$$
\begin{aligned}
& \bar{\pi}_{a m t}-\bar{\pi}_{j m t}-\left(F_{j m t}^{k}-F_{a m t}^{k}\right) \sum_{d=1}^{\tau} \beta^{d} \Lambda_{J m t d} \bar{\pi}_{J m t+d} \\
= & \bar{\pi}_{a m t}^{\prime}-\bar{\pi}_{j m t}^{\prime}-\left(F_{j m t}^{k}-F_{a m t}^{k}\right) \sum_{d=1}^{\tau} \beta^{d} \Lambda_{J m t d} \bar{\pi}_{J m t+d}^{\prime},
\end{aligned}
$$

for almost all $\left(w_{m t}, \ldots, w_{m t+\tau}\right)$. Consider (B5) for $j=J$. Because $\bar{\pi}_{J}(k, w)$ is known for all observed states $(k, w)$, we conclude that $\bar{\pi}_{a m t}=\bar{\pi}_{a m t}^{\prime}$ almost surely.

\section{B.2 Proof of Proposition 2}

Equation (31) is a linear regression equation, and $E\left[Z_{m t} u_{a j m t}\right]=0$ and $\operatorname{rank}\left(E\left[Z_{m t} \widetilde{X}_{a j m t}\right]\right)=P$ are the standard orthogonality and rank conditions, respectively, for parameter identification. 


\section{B.3 Proof of Lemma 1}

We omit the subscripts $i$ and $m$ to simplify notation. Suppose Assumption 4 holds.

(i) From the definition of $e^{h}\left(a, k, \omega_{t}, \omega_{t+1}^{*}\right)$,

$$
\begin{aligned}
& E\left[e^{h}\left(a, k, \omega_{t}, \omega_{t+1}^{*}\right) \mid \mathcal{I}_{t}\right]=E\left[\sum_{k^{\prime}} e^{h}\left(k^{\prime}, \omega_{t}, \omega_{t+1}^{*}\right) F^{k}\left(k^{\prime} \mid a, k, w_{t}\right) \mid \mathcal{I}_{t}\right] \\
= & E\left[\sum_{k^{\prime}}\left(\int_{\omega^{\prime}} h\left(k^{\prime}, \omega^{\prime}\right) d F^{\omega}\left(\omega^{\prime} \mid \omega_{t}\right)-h\left(k^{\prime}, \omega_{t+1}^{*}\right)\right) F^{k}\left(k^{\prime} \mid a, k, w_{t}\right) \mid \mathcal{I}_{t}\right] \\
= & \sum_{k^{\prime}} \int_{\omega^{\prime}} h\left(k^{\prime}, \omega^{\prime}\right) d F^{\omega}\left(\omega^{\prime} \mid \omega_{t}\right) F^{k}\left(k^{\prime} \mid a, k, w_{t}\right) \\
& -\sum_{k^{\prime}} \int_{\omega_{t+1}^{*}} h\left(k^{\prime}, \omega_{t+1}^{*}\right) d F^{\omega}\left(\omega_{t+1}^{*} \mid \omega_{t}\right) F^{k}\left(k^{\prime} \mid a, k, w_{t}\right) \\
= & 0 .
\end{aligned}
$$

(ii) By the law of iterated expectations,

$$
E\left[e^{h}\left(a, k, \omega_{t}, \omega_{t+1}^{*}\right) \mid z_{t}\right]=E\left[E\left[e^{h}\left(a, k, \omega_{t}, \omega_{t+1}^{*}\right) \mid \mathcal{I}_{t}\right] \mid z_{t}\right]=0
$$

where the second equality follows from (i).

Note also that, given that the time-t information set $\mathcal{I}_{t}$ includes current and past variables, Lemma 1 also implies that $E\left[e^{h}\left(a, k, \omega_{t}, \omega_{t+1}^{*}\right) \mid z_{t-d}\right]$, for all $a, k$ and any $d \geq 1$. In particular, $E\left[e^{h}\left(a, k, \omega_{t+d}, \omega_{t+d+1}^{*}\right) \mid z_{t}\right]$.

(iii) Next, fix $a$ and $k$, and simplify notation further by defining $e^{h}\left(a, k_{t}, \omega_{t}, \omega_{t+1}^{*}\right)=e_{t+1}^{h}$. Note that not only current and past states $(k, \omega)$ belong to the information set available to agents $\mathcal{I}_{t}$, but also past prediction errors. I.e., $\left\{e_{t}^{h}, e_{t-1}^{h}, \ldots, e_{1}^{h}\right\} \in \mathcal{I}_{t}$. We can then let $z_{t}=e_{t-d}^{h}$ for $d \geq 1$ and use result (ii) above to establish that $E\left[e_{t-d}^{h} e_{t}^{h}\right]=0$. Thus, expectational errors are serially uncorrelated.

\section{Proofs: Estimation and Inference}

\section{C.1 Proof of Lemma 2}

Given that $\left\{a_{i m t}, k_{i m t}: i=1, \ldots, N\right\}$ are i.i.d. conditional on $\omega_{m t}$, the first part of the Lemma (the almost sure convergence) follows by an immediate application of the Law of Large Numbers for exchangeable random variables (see Hall and Heyde (1980), p. 202, (7.1)).

The second part is obtained in three steps. First, Horvath and Yandell (1988) presents a Law of Iterated Logarithm (LIL) applied to both kernel and nearest neighbor estimators for conditional probabilities (see their Corollary 5.1). The i.i.d. sample in Horvath and Yandell (1988) can be 
replaced by the assumption that the sample is i.i.d. conditional on the common shocks following the arguments in Souza-Rodrigues $(2016) .^{2}$ The LIL then holds for almost all $\omega_{m t}$. Finally, it is straightforward to adapt the kernel regression results to simple frequency estimators (i.e., use simple indicator functions as kernel functions).

\section{C.2 Proof of Proposition 3}

Recall that $g_{m t}(\theta)=h\left(z_{m t}\right) u_{m t}\left(\theta, \delta_{m t}\right)$. Define the following functions:

$$
\widetilde{g}_{M}(\theta)=\frac{1}{M(T-\tau)} \sum_{m=1, t=1}^{M,(T-\tau)} g_{m t}(\theta)
$$

and

$$
\widetilde{Q}_{M}(\theta)=\widetilde{g}_{M}(\theta)^{\prime} \mathbf{W}_{M} \widetilde{g}_{M}(\theta) .
$$

The criterion function $\widetilde{Q}_{M}(\theta)$ is similar to $\widehat{Q}_{M}(\theta)$ but makes use of $\delta_{m t}$ instead of the estimator $\widehat{\delta}_{m t}$. I.e., $\widetilde{Q}_{M}(\theta)$ is an unfeasible GMM criterion function, while $\widehat{Q}_{M}(\theta)$ is feasible. The unfeasible estimator $\widetilde{\theta}_{M}$ (approximately) minimizes $\widetilde{Q}_{M}(\theta)$ over $\Theta$.

A straightforward application of Theorem 2.6 in Newey and McFadden (1994) proves that the unfeasible estimator $\widetilde{\theta}_{M}$ is a consistent estimator of $\theta_{0}$. To show that the feasible estimator $\widehat{\theta}_{M}$ is consistent as well, it suffices to show that $\widehat{Q}_{M}(\theta)$ converges in probability to $\widetilde{Q}_{M}(\theta)$ uniformly over $\Theta$. To do so, define the difference $v_{m t}=\widehat{g}_{m t}(\theta)-g_{m t}(\theta)$, and

$$
v_{M}(\theta)=\frac{1}{M(T-\tau)} \sum_{m=1, t=1}^{M,(T-\tau)} v_{m t}(\theta)
$$

Then,

$$
\begin{aligned}
\widehat{Q}_{M}(\theta) & =\left[\widetilde{g}_{M}(\theta)+v_{M}(\theta)\right]^{\prime} \mathbf{W}_{M}\left[\widetilde{g}_{M}(\theta)+v_{M}(\theta)\right] \\
& =\widetilde{Q}_{M}(\theta)+v_{M}(\theta)^{\prime} \mathbf{W}_{M} v_{M}(\theta)+2 \widetilde{g}_{M}(\theta)^{\prime} \mathbf{W}_{M} v_{M}(\theta)
\end{aligned}
$$

Given Condition 2(ii), it suffices to show that both $\widetilde{g}_{M}(\theta)$ and $v_{M}(\theta)$ converge to zero in probability uniformly over $\Theta$.

By Conditions 2(i),(iii),(v), and (vi), $\widetilde{g}_{M}(\theta)$ satisfies the uniform Weak Law of Large Numbers, and therefore converges in probability to zero uniformly over $\Theta$ as $M \rightarrow \infty$. Now consider $v_{M}(\theta)$. Note that

$$
v_{m t}=h\left(z_{m t}\right)\left(u_{m t}\left(\theta, \widehat{\delta}_{m t}\right)-u_{m t}\left(\theta, \delta_{m t}\right)\right)
$$

\footnotetext{
${ }^{2}$ Souza-Rodrigues (2016) establishes the asymptotic properties of the kernel regression estimator for crosssectional data in the presence of common shocks.
} 
and take a mean-value expansion of $u_{m t}\left(\theta, \widehat{\delta}_{m t}\right)$ about $\delta_{m t}$ :

$$
u_{m t}\left(\theta, \widehat{\delta}_{m t}\right)-u_{m t}\left(\theta, \delta_{m t}\right)=\nabla_{\delta} u_{m t}\left(\theta, \delta_{m t}^{*}\right)\left(\widehat{\delta}_{m t}-\delta_{m t}\right)
$$

where $\delta_{m t}^{*}$ lies between $\widehat{\delta}_{m t}$ and $\delta_{m t}$. Next, note that

$$
\begin{aligned}
E\left[\sup _{\theta \in \Theta}\left\|v_{M}(\theta)\right\|\right] & \leq \frac{1}{M(T-\tau)} \sum_{m=1, t=1}^{M,(T-\tau)} E\left[\sup _{\theta \in \Theta}\left\|h\left(z_{m t}\right) \nabla_{\delta} u_{m t}\left(\theta, \delta_{m t}^{*}\right)\right\|\left\|\widehat{\delta}_{m t}-\delta_{m t}\right\|\right] \\
& \leq \frac{B}{M(T-\tau)} \sum_{m=1, t=1}^{M,(T-\tau)} E\left[\left\|\widehat{\delta}_{m t}-\delta_{m t}\right\|^{2}\right]
\end{aligned}
$$

where the second inequality follows from the Cauchy-Schwarz inequality and Condition 2(vii). Because $\left\|\widehat{\delta}_{m t}-\delta_{m t}\right\| \stackrel{p}{\rightarrow} 0$, as $N \rightarrow \infty$, by Lemma 2, we have that $E\left[\left\|\widehat{\delta}_{m t}-\delta_{m t}\right\|^{2}\right]=o(1)$, and, so, the right-hand-side of (C3) converges to zero as $N \rightarrow \infty$ for all $M$ and $T$. We conclude that

$$
\sup _{\theta \in \Theta}\left\|\widehat{Q}_{M}(\theta)-\widetilde{Q}_{M}(\theta)\right\| \stackrel{p}{\rightarrow} 0, \text { as }(M, N) \rightarrow \infty
$$

\section{C.3 Proof of Proposition 4 .}

By standard arguments (see Theorem 3.2 in Newey and McFadden (1994), the unfeasible estimator $\widetilde{\theta}_{M}$ satisfies

$$
\widetilde{\theta}_{M}-\theta_{0}=-\left[\mathbf{G}^{\prime} \mathbf{W} \mathbf{G}\right]^{-1} \mathbf{G}^{\prime} \mathbf{W} g\left(\theta_{0}\right)+o_{p}(1 / \sqrt{M})
$$

and is asymptotically normal,

$$
\sqrt{M}\left(\widetilde{\theta}_{M}-\theta_{0}\right) \stackrel{p}{\rightarrow} N(0, \mathbf{V})
$$

under Conditions 3(i)-(iv). The asymptotic distribution of the feasible estimator $\widehat{\theta}_{M}$ is the same as the asymptotic distribution of the unfeasible $\widetilde{\theta}_{M}$ provided

$$
\left\|\widehat{\theta}_{M}-\widetilde{\theta}_{M}\right\|=o_{p}\left(\frac{1}{\sqrt{M}}\right) .
$$

From (C4), it is clear that

$$
\widetilde{\theta}_{M}-\widehat{\theta}_{M}=\left[\mathbf{G}^{\prime} \mathbf{W} \mathbf{G}\right]^{-1} \mathbf{G}^{\prime} \mathbf{W} v_{M}\left(\theta_{0}\right)+o_{p}(1 / \sqrt{M})
$$

So,

$$
\left\|\widehat{\theta}_{M}-\widetilde{\theta}_{M}\right\| \leq\left\|\left[\mathbf{G}^{\prime} \mathbf{W} \mathbf{G}\right]^{-1}\right\|\|\mathbf{G}\|\|\mathbf{W}\|\left\|v_{M}\left(\theta_{0}\right)\right\|+o_{p}(1 / \sqrt{M}) .
$$


Note that

$$
E\left[\left\|v_{M}\left(\theta_{0}\right)\right\|\right] \leq \frac{B}{M(T-\tau)} \sum_{m=1, t=1}^{M,(T-\tau)}\left(E\left[\left\|\widehat{\delta}_{m t}-\delta_{m t}\right\|^{2}\right]\right)^{1 / 2}
$$

by Condition 2(vii). Because $E\left[\left\|\widehat{\delta}_{m t}-\delta_{m t}\right\|^{2}\right]=O\left(\frac{\log \log N}{N}\right)$, by Lemma 2 , we have that $\left\|v_{M}\left(\theta_{0}\right)\right\|=$ $O_{p}\left(\sqrt{\frac{\log \log N}{N}}\right)$, which implies

$$
\sqrt{M}\left\|\widehat{\theta}_{M}-\widetilde{\theta}_{M}\right\|=O_{p}\left(\sqrt{\frac{M \log \log N}{N}}\right)=o_{p}(1)
$$

provided $\frac{M \log \log N}{N} \rightarrow 0$

\section{C.4 Additional Result}

The next lemma provides a result that is used in Proposition 1. Proposition 1 claims that, for a known function $f$ of $\delta_{m t}^{\tau}=\left(\delta_{m t}, \ldots, \delta_{m t+\tau}\right)$, quantities of the type $E\left[h\left(z_{m t}\right) f\left(\delta_{m t}^{\tau}\right)\right]$ can be recovered from the data. (More specifically, $f\left(\delta_{m t}^{\tau}\right)$ in the proof of Proposition 1 corresponds to the term in parenthesis on the LHS of equation (B4).)

Lemma C1. Suppose the vector $\left(w_{m t}, z_{m t}\right)$ is i.i.d. across markets m. Assume

$$
E\left[\left\|h\left(z_{m t}\right) \nabla_{\delta} f\left(\delta_{m t}^{\tau}\right)\right\|^{2}\right] \leq C<\infty
$$

Then

$$
\frac{1}{M} \sum_{m=1}^{M} h\left(z_{m t}\right) f\left(\widehat{\delta}_{m t}^{\tau}\right) \stackrel{p}{\rightarrow} E\left[h\left(z_{m t}\right) f\left(\delta_{m t}^{\tau}\right)\right]
$$

as $(M, N) \rightarrow \infty .^{3}$

Proof. First, note that

$$
\frac{1}{M} \sum_{m=1}^{M} h\left(z_{m t}\right) f\left(\widehat{\delta}_{m t}^{\tau}\right)=\frac{1}{M} \sum_{m=1}^{M} h\left(z_{m t}\right) f\left(\delta_{m t}^{\tau}\right)+\frac{1}{M} \sum_{m=1}^{M} h\left(z_{m t}\right)\left[f\left(\widehat{\delta}_{m t}^{\tau}\right)-f\left(\delta_{m t}^{\tau}\right)\right]
$$

The first term on the right-hand-side converges in probability to $E\left[h\left(z_{m t}\right) f\left(\delta_{m}\right)\right]$ as $M \rightarrow \infty$ by the Weak Law of Large Numbers. Applying a mean-value expansion on the second term, we get

$$
\frac{1}{M} \sum_{m=1}^{M} h\left(z_{m t}\right)\left[f\left(\widehat{\delta}_{m t}^{\tau}\right)-f\left(\delta_{m t}^{\tau}\right)\right]=\frac{1}{M} \sum_{m=1}^{M} h\left(z_{m t}\right) \nabla_{\delta} f\left(\delta_{m t}^{\tau *}\right)\left[\widehat{\delta}_{m t}^{\tau}-\delta_{m t}^{\tau}\right]
$$

\footnotetext{
${ }^{3}$ The same result applies if $\left(w_{m t}, z_{m t}\right)$ is stationary and ergodic, if we average the term $\left[h\left(z_{m t}\right) f\left(\widehat{\delta}_{m t}^{\tau}\right)\right]$ over $T-\tau$ time periods, and if we take $(T, N) \rightarrow \infty$.
} 
where $\delta_{m t}^{\tau *}$ lies between $\widehat{\delta}_{m t}^{\tau}$ and $\delta_{m t}^{\tau}$. Next, note that

$$
\begin{aligned}
E\left[\left\|h\left(z_{m t}\right) \nabla_{\delta} f\left(\delta_{m t}^{\tau *}\right)\left[\widehat{\delta}_{m t}^{\tau}-\delta_{m t}^{\tau}\right]\right\|\right] & \leq\left(E\left[\left\|h\left(z_{m t}\right) \nabla_{\delta} f\left(\delta_{m t}^{\tau *}\right)\right\|^{2}\right] E\left[\left\|\widehat{\delta}_{m t}^{\tau}-\delta_{m t}^{\tau}\right\|^{2}\right]\right)^{1 / 2} \\
& \leq C\left(E\left[\left\|\widehat{\delta}_{m t}^{\tau}-\delta_{m t}^{\tau}\right\|^{2}\right]\right)^{1 / 2}
\end{aligned}
$$

where the first inequality follows from the Cauchy-Schwarz inequality, and the second inequality from the regularity condition $E\left[\left\|h\left(z_{m t}\right) \nabla_{\delta} f\left(\delta_{m t}^{\tau}\right)\right\|^{2}\right] \leq C<\infty$. By Lemma $2, E\left[\left\|\widehat{\delta}_{m t}^{\tau}-\delta_{m t}^{\tau}\right\|^{2}\right]$ converges to zero as $N \rightarrow \infty$, which implies

$$
\frac{1}{M} \sum_{m=1}^{M} h\left(z_{m t}\right)\left[f\left(\widehat{\delta}_{m t}^{\tau}\right)-f\left(\delta_{m t}^{\tau}\right)\right] \stackrel{p}{\rightarrow} 0, \text { as } N \rightarrow \infty, \text { for all } M
$$

\section{The Standard CCP Estimator}

Here we explain the standard CCP approach implemented in the Monte Carlo experiment to estimate the model parameters. By "standard," we mean involving a full specification of how all state variables evolve, and not relying on Euler equations. Following Hotz and Miller (1993), this CCP approach avoids the computational burden of solving the dynamic problem within the estimation algorithm associated with Rust's (1987) nested fixed point approach.

The estimation here follows Section 2 of Kalouptsidi, Scott, and Souza-Rodrigues (2019) and we refer readers to it for details. Estimation begins by estimating choice probabilities conditional on individual states and the modeled exogenous state variable, i.e., $p(k, w)$. Let $F_{b}$ represent the stochastic matrix for observable state variables $(k, w)$ conditional on buying the product, and let $F_{n b}$ represent the stochastic matrix when the action is not buying the product. Kalouptsidi, Scott, and Souza-Rodrigues (2019) shows that

$$
\pi_{b}=A \pi_{n b}+\boldsymbol{b}
$$

where $A=\left(I-\beta F_{b}\right)\left(I-\beta F_{n b}\right)^{-1}$ and $\boldsymbol{b}=A \psi_{n b}-\psi_{b}$, where $\psi_{a}$ stacks $\psi_{a}(p(k, w))$ across all values of $(k, w)$.

We estimate the payoff parameters $\theta$ using a Minimum Distance estimator, i.e., by minimizing the L2 norm of

$$
\pi_{b}(\theta)-A \pi_{n b}(\theta)-\boldsymbol{b}
$$


Given the parameterization, this is achieved by a linear regression of the vector $\boldsymbol{b}$ on the matrix

$$
[(\mathbf{1}-A \boldsymbol{k}), \boldsymbol{w}]
$$

where $\mathbf{1}$ is a vector of ones, $\boldsymbol{k}$ is a dummy vector equal to one in states where the good is owned, and $\boldsymbol{w}$ is the vector of prices. ${ }^{4}$

\section{E Monte Carlo: Counterfactual}

In the Monte Carlo presented in Section 6, we consider only the estimation of the parameters of agents' utility function. Typically, applied researchers are also interested in the outcomes of policy simulations or counterfactuals. In this section, we extend the Monte Carlo experiment in Section 6 to study counterfactual simulations. Specifically, we consider how the biases in the parameter estimates pass through to biases in countefactuals, but first, we must consider the question of how to do counterfactuals within the ECCP framework.

Much of the ECCP approach's appeal comes from the fact that it takes seriously the possibility that the econometrician might be facing important measurement issues; e.g., some market-level state variables might not be observed, and/or it might be difficult to specify how they evolve. However, when doing counterfactuals, researchers typically solve for a new equilibrium of the model, which normally involves fully specifying all the relevant state variables and how they evolve. Thus, prima facie, ECCP estimation seems to be at odds with doing counterfactual simulations.

A counterfactual is a function of the model parameters, and sometimes that function does not depend (or depends only minimally) on the presence of unobservable variables or on the precise specification of how state variables evolve. Therefore, the modeling issues that motivate the ECCP approach need not undermine the use of parameter estimates for counterfactual analysis. De Groote and Verboven (2019) provide a clear example. They use an ECCP estimator to estimate the rate of time discounting of Belgian households in deciding whether to install solar photovoltaic systems (the ECCP estimator allows them to flexibly include demand shocks and avoid specifying a process for how government policy evolved). They find that households' estimated discount rate is considerably lower than the interest rate that the Belgian government can borrow at. As they argue, this disparity means that it would be more cost effective for the government to support solar PV installations with up-front payments, rather than the ongoing payments that the government actually used. This conclusion follows intuitively from the disparity in discount rates and plausibly is not affected in an important way by how government policy and unobservable states evolve. The conclusion, however, may be highly sensitive to biases in the estimation of the discount factor. In

\footnotetext{
${ }^{4}$ Note that one can estimate the model parameters either by minimizing the distance between $\boldsymbol{b}$ and $\pi_{b}(\theta)-$ $A \pi_{n b}(\theta)$, or by minimizing the distance between the (nonparametrically) estimated CCP, $p$, and the CCP generated by the model, $p(\theta)$. See Pesendorfer and Schmidt-Dengler (2008).
} 
other words, the estimation of a mis-specified model may crucially affect policy recommendations.

In what follows, we show that some counterfactuals - specifically, long-run demand elasticities from our durable good demand model - are robust to the omission of unobservable state variables that are present in the data generating process. Or more specifically, long-run demand elasticities are well approximated by a model in which we set $\xi$ at its long-run mean (i.e., $\xi=0)$. Furthermore, we show that the biases that result from leaving the unobservable shocks out of the counterfactual simulations can be smaller than the biases that result from using an estimation approach that is not robust to their presence.

We perform both a real and feasible counterfactuals for the durable good demand model. Our real counterfactuals take the parameter estimates from various estimations presented in Section 6 and plug them into a counterfactual that uses the true data generating process (notably including the true law of motion for the unobservable demand shock $\left.\xi_{m t}\right)$. That is, the real counterfactuals rely on our understanding of an unobservable that an econometrician who was not simulating the data would not have access to. Our feasible counterfactuals, in contrast, simulate a simple model that an econometrician could easily implement: a model that sets $\xi=0 .{ }^{5}$

The counterfactual we consider is an increase in the mean price level (formally, we increase $\gamma_{0}$ by .01; see Table 1 in Section 6 ), and we calculate the long-run change in the demand level. That is, we calculate the unconditional probability of purchase $\operatorname{Pr}(a=b)$ in the steady state after solving the consumer's dynamic problem. We present this counterfactual in the form of a long-run demand elasticity, i.e., the ratio of the percentage change in the probability of purchase to the percentage change in the long-run price.

Table E1 shows the counterfactuals from the ECCP (OLS and IV) and standard CCP estimators based on the parameter estimates from the above simulations with $M=160$ and $T=160$. A first observation is that the real and feasible counterfactuals at the true parameters differ by a factor of about $10 \%$. Second, consistent with the biases in the underlying parameter estimates, we find that the ECCP IV estimates yield very little bias in the counterfactuals relative to the true values while the other estimators result in substantially biased counterfactuals. Furthermore, the biases in the long-run elasticities from the OLS and standard CCP estimators (whether we consider the real or feasible versions) are larger than the gap between the real and feasible estimators.

Evidently, counterfactuals are not always robust to setting $\xi$ at its unconditional mean. The broader point we make in this section is that robustness to the presence of unobserved shocks can be assessed through a procedure similar to what we do here. That is, when researchers are concerned about the presence of unobservables, they might adopt a robust estimation approach that delivers consistent estimates of important parameters despite the unobservables. Then, when it comes to counterfactual simulations, they can perform the simulation in several ways to assess

\footnotetext{
${ }^{5}$ To solve the feasible counterfactual, we need to specify how the exogenous state variable $w_{m t}$ evolves. We consider the residual from the pricing equation (39) as the econometrician can measure it. I.e., $w=\gamma_{0}+\gamma_{1} z+\nu$, where $\nu=\gamma_{2} \xi+\varepsilon^{w}$. So, we calculate the true evolution of $\nu$ given the underlying processes and assume the econometrician is able to estimate it.
} 
whether and how the results of interest might be sensitive to the presence of unobservables and how they evolve.

Table E1: Sample size, structure and bias

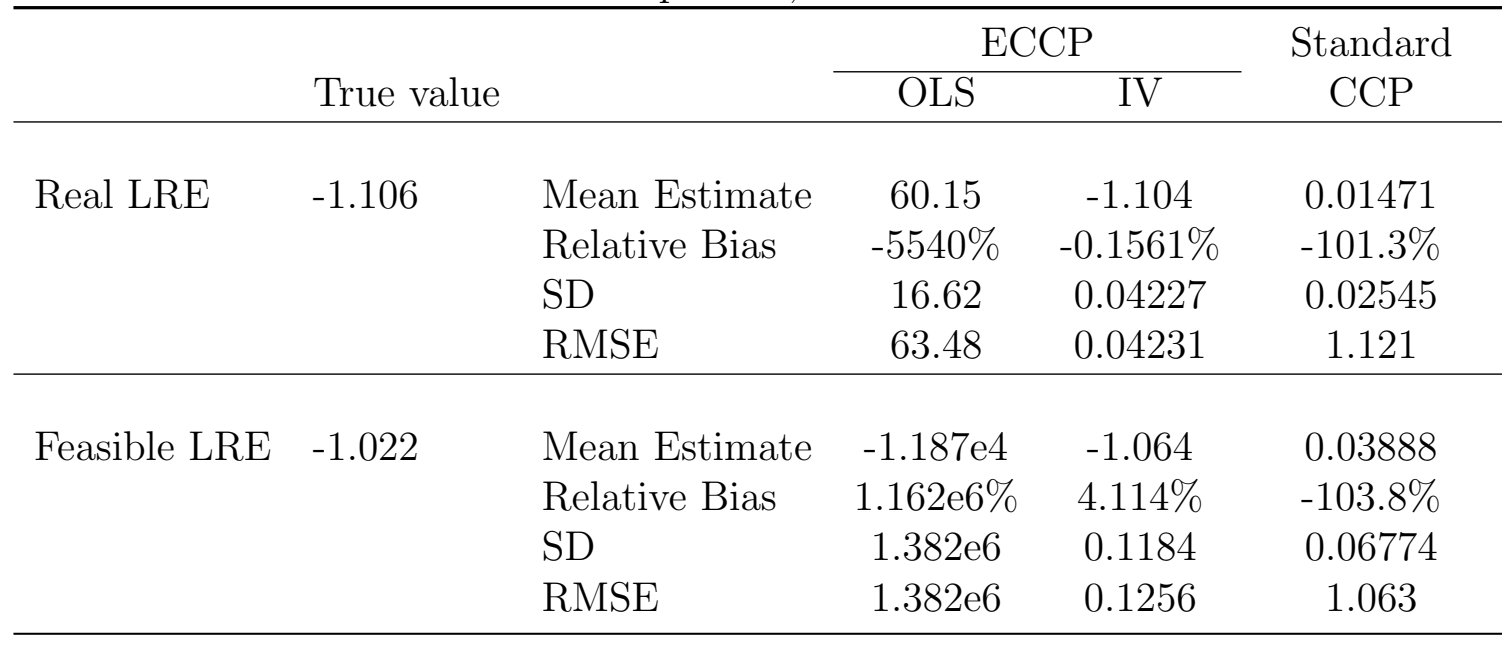

Notes: 5000 replications with sample structure $M=T=160$. SD is the standard deviation across replications. RMSE is root-mean squared error.

Relative Bias is bias as percentage of the true parameter.

\section{References}

Arcidiacono, P., And R. A. Miller (2019): "Nonstationary dynamic models with finite dependence," Quantitative Economics, 10, 853-890.

De Groote, O., and F. Verboven (2019): "Subsidies and Time Discounting in New Technology Adoption: Evidence from Solar Photovoltaic Systems," American Economic Review, 109(6), $2137-72$.

Hall, P., And C. C. Heyde (1980): Martingale limit theory and its application. Academic press.

Horvath, L., And B. S. YAndell (1988): "Asymptotics of conditional empirical processes," Journal of Multivariate Analysis, 26(2), 184-206.

Hotz, V. J., and R. A. Miller (1993): "Conditional Choice Probabilities and the Estimation of Dynamic Models," Review of Economic Studies, 60(3), 497-529.

Kalouptsidi, M., P. T. Scott, And E. A. Souza-Rodrigues (2019): "Identification of Counterfactuals in Dynamic Discrete Choice Models," Quantitative Economics, forthcoming.

Newey, W. K., and D. McFadden (1994): "Large Sample Estimation and Hypothesis Testing," Handbook of Econometrics, 4, 2111-2245. 
Pesendorfer, M., And P. Schmidt-Dengler (2008): "Asymptotic Least Squares Estimators for Dynamic Games," The Review of Economic Studies, 75(3), 901-928.

Ransom, T. (2019): "Labor Market Frictions and Moving Costs of the Employed and Unemployed," Discussion Paper 12139, IZA Institute of Labor Economics.

Rust, J. (1987): "Optimal Replacement of GMC Bus Engines: an Empirical Model of Harold Zurcher," Econometrica, 55(5), 999-1033.

SouzA-Rodrigues, E. A. (2016): "Nonparametric Regression with Common Shocks," Econometrics, $4(3), 36$. 